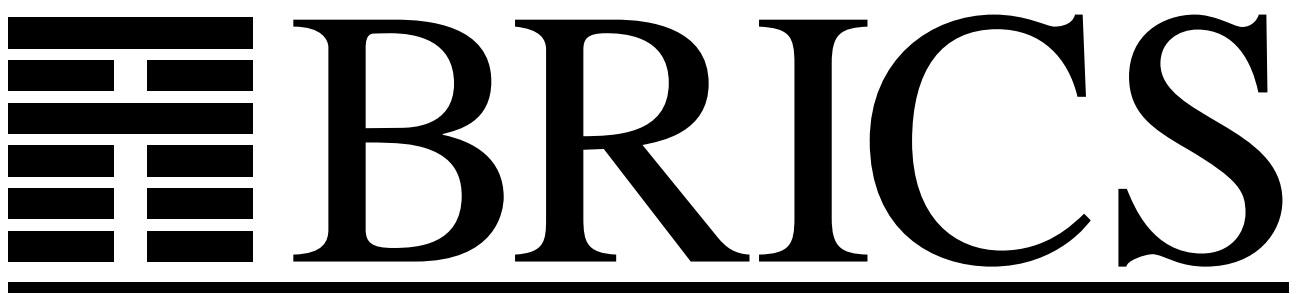

Basic Research in Computer Science

\title{
Denotational Aspects of Untyped Normalization by Evaluation
}

\author{
Andrzej Filinski \\ Henning Korsholm Rohde
}

RS-05-4 
Copyright (c) 2005, Andrzej Filinski \& Henning Korsholm Rohde. BRICS, Department of Computer Science University of Aarhus. All rights reserved.

Reproduction of all or part of this work is permitted for educational or research use on condition that this copyright notice is included in any copy.

See back inner page for a list of recent BRICS Report Series publications. Copies may be obtained by contacting:

\author{
BRICS \\ Department of Computer Science \\ University of Aarhus \\ Ny Munkegade, building 540 \\ DK-8000 Aarhus C \\ Denmark \\ Telephone: +4589423360 \\ Telefax: $\quad+4589423255$ \\ Internet: BRICS@brics.dk
}

BRICS publications are in general accessible through the World Wide Web and anonymous FTP through these URLs:

http://www.brics.dk

ftp: //ftp.brics.dk

This document in subdirectory RS / 05 / 4 / 


\title{
Denotational Aspects of Untyped Normalization by Evaluation*
}

\author{
(Extended version, with detailed proofs) \\ Andrzej Filinski \\ DIKU, University of Copenhagen, Denmark \\ andrzej@diku.dk \\ Henning Korsholm Rohde \\ BRICS; University of Aarhus, Denmark \\ hense@brics.dk
}

February 2005

\begin{abstract}
We show that the standard normalization-by-evaluation construction for the simply-typed $\lambda_{\beta \eta}$-calculus has a natural counterpart for the untyped $\lambda_{\beta}$-calculus, with the central type-indexed logical relation replaced by a "recursively defined" invariant relation, in the style of Pitts. In fact, the construction can be seen as generalizing a computational-adequacy argument for an untyped, call-by-name language to normalization instead of evaluation.

In the untyped setting, not all terms have normal forms, so the normalization function is necessarily partial. We establish its correctness in the senses of soundness (the output term, if any, is in normal form and $\beta$-equivalent to the input term); identification ( $\beta$-equivalent terms are mapped to the same result); and completeness (the function is defined for all terms that do have normal forms). We also show how the semantic construction enables a simple yet formal correctness proof for the normalization algorithm, expressed as a functional program in an ML-like, call-by-value language.

Finally, we generalize the construction to produce an infinitary variant of normal forms, namely Böhm trees. We show that the three-part characterization of correctness, as well as the proofs, extend naturally to this generalization.
\end{abstract}

\footnotetext{
* Extended version of an article to appear in RAIRO - Theoretical Informatics and Applications. An earlier version appeared in the proceedings of FOSSACS 2004 [FR04, FR03].

${ }^{\dagger}$ Basic Research in Computer Science (www.brics.dk),

funded by the Danish National Research Foundation.
} 


\section{Contents}

1 Introduction 3

1.1 Reduction-based and reduction-free normalization $\ldots \ldots \ldots \ldots$

1.2 Normalization by evaluation . . . . . . . . . . . . . . . 3

1.3 The Berger-Schwichtenberg normalization algorithm . . . . . . . . 4

1.4 A tentative algorithm for untyped terms . . . . . . . . . . . 4

1.5 Related work . . . . . . . . . . . . . . . . . . . . 5

$\begin{array}{lll}2 & \text { A semantic normalization construction } & \mathbf{7}\end{array}$

2.1 Syntax and semantics of the untyped $\lambda$-calculus $\ldots \ldots \ldots \ldots \ldots$

2.2 Output-term generation $\ldots \ldots \ldots \ldots$

2.3 A residualizing model . . . . . . . . . . . . . . . . . . . . . . 9

3 Correctness of the construction $\quad 10$

3.1 Correctness of the wrappers . . . . . . . . . . . . . . . . 10

3.2 Adequacy of the residualizing model . . . . . . . . . . . . . . 11

3.3 Correctness of the normalization function . . . . . . . . . . . 15

4 An implementation of the construction $\quad 16$

4.1 Syntax and semantics of an ML-like call-by-value language . . . . . . . 16

4.2 The normalization algorithm . . . . . . . . . . . . . . . . . 19

5 A generalization to Böhm trees 25

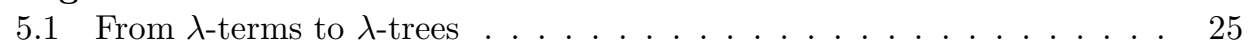

5.2 A semantic Böhm-tree construction . . . . . . . . . . . . . . 28

5.3 Correctness of the construction . . . . . . . . . . . . . . . . 29

5.4 An implementation of the construction . . . . . . . . . . 36

5.5 Observing Böhm trees . . . . . . . . . . . . . . . . . . . . . 40

5.5.1 Computations with infinite results . . . . . . . . . 40

5.5.2 Observing $\lambda$-trees . . . . . . . . . . . . . . . . . . 41

5.5.3 Implementing tree-observations in ML . . . . . . . . . . . 42

6 Conclusions and perspectives 43

A Existence of invariant relations $\quad 45$

B Existence of isomorphisms $\quad 47$

B.1 Isomorphisms for the redualizing model . . . . . . . . . . . . 47

B.2 Isomorphisms for Böhm trees . . . . . . . . . . . . . . . . . 49 


\section{Introduction}

\subsection{Reduction-based and reduction-free normalization}

Traditional accounts of term normalization are based on a directed notion of reduction (such as $\beta$-reduction), which can be applied anywhere within a term. A term is said to be a normal form if no reductions can be performed on it. If the reduction relation is confluent, normal forms are uniquely determined, so normalization is a (potentially partial) function on terms. Some terms (such as $\Omega$ ) may not have normal forms at all; or a particular reduction strategy (such as normal-order reduction) may be required to guarantee arrival at a normal form when one exists; such a strategy is called complete. There is a very large body of work dealing with normalization in reduction-based settings.

However, in recent years, a rather different notion of normalization has emerged, so-called reduction-free normalization. As the name suggests, it is not based on a directed notion of reduction, but rather on an undirected notion of term equivalence. Equivalence may be defined as simply the reflexive-transitive-symmetric closure of an existing reduction relation, but it does not have to be: any congruence relation on terms may be used. The task is then to define a normalization function on terms, such that the output of the function is equivalent to the input, and such that any two equivalent terms are mapped to identical outputs [CD97].

For some notions of equivalence (such as $\beta$-convertibility of untyped lambdaterms), it is actually impossible to define a computable, total normalization function with both of these properties; we must thus accept that the normalization function may be partial. However, even in that case, we can impose a completeness constraint: if we have an independent syntactic characterization of acceptable normal forms, we can require that the function both produce terms in this form as output, and that it be defined on all terms equivalent to a normal form.

\subsection{Normalization by evaluation}

A particularly natural way of obtaining a reduction-free normalization function is known as normalization by evaluation (NBE), based on the following idea: Suppose we can construct a denotational model of the term syntax (i.e., such that equivalent terms have the same denotation), with the property that a syntactic representation of any normal-form term can be extracted from its denotation; such a model is called residualizing. Then the normalization function can be expressed simply as a compositional interpretation in the model, followed by extraction.

A priori, such a normalization function is not necessarily effectively computable. It can be given a computational interpretation if the denotational model is constructed in intuitionistic set theory [CD97], but this gets somewhat complicated for domaintheoretic models, especially those involving reflexive domains. In such cases, it is often easier to establish that the constructions are effective by showing that they can expressed as images of program terms in a language for which the domain-theoretic semantics is already known to be computationally adequate.

(It should be noted that the term NBE is also sometimes used for a related concept, based on reducing - usually in a compositional way - the normalization problem, which may in general involve open terms of higher type, to an evaluation problem, which 
involves normalization of only closed terms of base type. The required transformation is often syntactically related to the model-based construction above, but the model itself is not made explicit; and in fact, the subsequent evaluation process may still be specified entirely in terms of reductions.)

\subsection{The Berger-Schwichtenberg normalization algorithm}

Perhaps the best-known NBE algorithm is due to Berger and Schwichtenberg [BS91]. It finds $\beta \eta$-long normal forms of simply-typed $\lambda$-terms. We present here its outline, glossing over inessential details.

Types are of the form $\tau::=b \mid \tau_{1} \rightarrow \tau_{2}$. A natural set-theoretic model interprets each base type $b$ as some set, and the function type as the set of all functions between the interpretations of the types, i.e., $\llbracket \tau_{1} \rightarrow \tau_{2} \rrbracket=\llbracket \tau_{1} \rrbracket \rightarrow \llbracket \tau_{2} \rrbracket$. For a type assignment

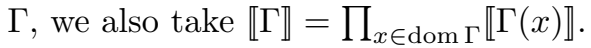

Let $\Lambda$ be the set of syntactic $\lambda$-terms (written with explicit constructors for emphasis) over a set of variables $\mathrm{V}$. For a well-typed term $\Gamma \vdash m: \tau$, we can then express its semantics $\llbracket m \rrbracket \in \llbracket \Gamma \rrbracket \rightarrow \llbracket \tau \rrbracket$ as follows:

$$
\begin{aligned}
\llbracket \operatorname{VAR}(x) \rrbracket \rho & =\rho(x) \\
\llbracket \operatorname{LAM}\left(x^{\tau}, m_{0}\right) \rrbracket \rho & =\lambda a^{\llbracket \tau \rrbracket} \cdot \llbracket m_{0} \rrbracket \rho[x \mapsto a] \\
\llbracket \operatorname{APP}\left(m_{1}, m_{2}\right) \rrbracket \rho & =\llbracket m_{1} \rrbracket \rho\left(\llbracket m_{2} \rrbracket \rho\right)
\end{aligned}
$$

It is easy to check that such a model is sound for conversion, i.e., that when $m \leftrightarrow_{\beta \eta} m^{\prime}$, then $\llbracket m \rrbracket=\llbracket m^{\prime} \rrbracket$.

Consider now a model where all base types are interpreted as the set of (open) syntactic $\lambda$-terms, i.e., $\llbracket b \rrbracket=\Lambda$ for all $b$. In this model, we can define a pair of typeindexed function families - reification, $\downarrow^{\tau}: \llbracket \tau \rrbracket \rightarrow \Lambda$, and reflection, $\uparrow^{\tau}: \Lambda \rightarrow \llbracket \tau \rrbracket-$ by mutual induction on the type index $\tau$ :

$$
\begin{array}{ll}
\downarrow^{b} l=l & \downarrow^{\tau_{1} \rightarrow \tau_{2}} f=\operatorname{LAM}\left(x^{\tau_{1}}, \downarrow^{\tau_{2}}\left(f\left(\uparrow^{\tau_{1}} \operatorname{VAR}(x)\right)\right)\right) \quad(x \text { "fresh") } \\
\uparrow^{b} l=l & \uparrow^{\tau_{1} \rightarrow \tau_{2}} l=\lambda a^{\llbracket \tau_{1} \rrbracket} \cdot \uparrow^{\tau_{2}}\left(\operatorname{APP}\left(l, \downarrow^{\tau_{1}} a\right)\right)
\end{array}
$$

For simplicity, let us only consider normal forms of closed terms. Then reification can serve directly as the extraction function: one can check that, for a term $\vdash m: \tau$ in $\beta \eta$ long normal form, $\downarrow^{\tau}(\llbracket m \rrbracket \emptyset) \leftrightarrow_{\alpha} m$. Hence, by soundness of the model, for any term $m^{\prime}$ with $m^{\prime} \leftrightarrow \leftrightarrow_{\eta} m, \downarrow^{\tau}\left(\llbracket m^{\prime} \rrbracket \emptyset\right)=\downarrow^{\tau}(\llbracket m \rrbracket \emptyset) \leftrightarrow_{\alpha} m \leftrightarrow_{\beta \eta} m^{\prime}$. Alternatively, one can show the latter property directly, for an arbitrary $m^{\prime}$. Either way, the typical proof ultimately involves a logical-relations argument, even if this argument is pushed entirely into a standard result about the syntax (namely, that every well-typed term has a $\beta \eta$-long normal form). The latter approach, however, generalizes better, especially to systems where not all terms have normal forms.

\subsection{A tentative algorithm for untyped terms}

In an untyped (or, more accurately, unityped) setting, we may hope to get a residualizing model by interpreting the single type of terms as a domain $D=\Lambda+(D \rightarrow D)$. (Again, we gloss over domain-theoretic subtleties for expository purposes.) We can 
then define variants of reification, $\downarrow: D \rightarrow \Lambda$, and reflection, $\uparrow: \Lambda \rightarrow D$, roughly analogous to the simply-typed case:

$$
\begin{aligned}
& \downarrow d=\text { case } d \text { of } \begin{cases}i n_{1}(l) & \rightarrow l \\
i n_{2}(f) & \rightarrow \operatorname{LAM}(x, \downarrow(f(\uparrow(\operatorname{VAR}(x))))) \quad(x \text { "fresh" })\end{cases} \\
& \uparrow l=i n_{1}(l) .
\end{aligned}
$$

Note that reification is now defined by general recursion, rather than induction. We can also construct an interpretation, $\llbracket m \rrbracket \in(\mathrm{V} \rightarrow D) \rightarrow D$, by

$$
\begin{aligned}
\llbracket \operatorname{VAR}(x) \rrbracket \rho & =\rho(x) \\
\llbracket \operatorname{LAM}\left(x, m_{0}\right) \rrbracket \rho & =i n_{2}\left(\lambda d . \llbracket m_{0} \rrbracket \rho[x \mapsto d]\right) \\
\llbracket \operatorname{APP}\left(m_{1}, m_{2}\right) \rrbracket \rho & =\text { case } \llbracket m_{1} \rrbracket \rho \text { of }\left\{\begin{array}{l}
i n_{1}(l) \rightarrow \uparrow\left(\operatorname{APP}\left(l, \downarrow\left(\llbracket m_{2} \rrbracket \rho\right)\right)\right) \\
i n_{2}(f) \rightarrow f\left(\llbracket m_{2} \rrbracket \rho\right) .
\end{array}\right.
\end{aligned}
$$

Here, reflection is performed "on demand": when application needs a semantic function, but $\llbracket m_{1} \rrbracket \rho$ is a piece of syntax, it is reflected just enough to allow the application to be performed.

Again, it can be checked that $\beta$-convertible terms have the same denotation. It is also fairly easy to verify that, for a closed $m$ in $\beta$-normal form, $\downarrow(\llbracket m \rrbracket \emptyset) \leftrightarrow_{\alpha} m$. What is not obvious at all, however, is that when $\downarrow\left(\llbracket m^{\prime} \rrbracket \emptyset\right)=m$ for a general $m^{\prime}$, then $m^{\prime}$ must be syntactically $\beta$-convertible to a normal form. Indeed, the problem is a generalization of the usual computational-adequacy problem for a denotational semantics of a functional language: if the denotation of a closed term is not $\perp$ (undefined), must the term then evaluate to a value?

For a simply typed language, PCF, adequacy of the natural domain-theoretic semantics was shown by Plotkin, using a logical-relations argument [Plo77]. Pitts showed that essentially the same argument applies to an untyped language, except that the central relation is no longer constructed by induction on types, but as a solution of a more general "relation equation"; he also showed a general method for solving such equations, yielding invariant relations [Pit93].

In this paper, we first formalize the construction of the normalization function from above, addressing especially the issues of potential divergence and generation of fresh variable names (Section 2). We then show correctness of this function by a generalized computational-adequacy construction (Section 3) and how the domain-theoretic analysis directly validates a functional program implementing the construction (Section 4). Finally, we show how the construction can be generalized naturally to Böhm trees (Section 5).

\subsection{Related work}

The closest related work to ours is probably the NBE-based (in the alternate, reductionoriented sense) algorithm for untyped $\beta$-normalization proposed by Aehlig and Joachimski [AJ04]. However, while the functional programs ultimately derived from the analyses are quite similar, the correctness arguments are completely different: theirs are based on syntactic concepts and results from higher-order rewriting theory, rather than on the domain-theoretic constructions underlying ours. 
We believe that the domain-theoretic approach enables a more direct and precise correctness proof for the normalizer, as actually implemented. In Aehlig and Joachimski's work, the abstract algorithm is expressed as a small-step operational semantics for a specialized, two-level $\lambda$-calculus with named bound variables; whereas the actual normalization program is formulated as a compositional interpreter in Haskell, using de Bruijn indices for bound variables, and a reflexive type for the meanings of highertyped terms. It thus remains a potentially significant task to verify that the concrete Haskell program correctly implements the abstract algorithm. On the other hand, formally relating the domain-theoretic constructions in the model-based normalizer to the functional terms implementing them, is completely straightforward.

An untyped, reduction-based NBE-like algorithm can also be found in disguise in Grégoire and Leroy's work [GL02], whose focus is on compilation. Their concrete algorithm of strong reduction (i.e., $\beta$-normalization) by iterated symbolic weak reduction (akin to $\uparrow$ and $\llbracket \cdot \rrbracket$ ) and readback (akin to $\downarrow$ ) is ultimately quite similar to ours. Their algorithm also handles several language extensions, such as inductive datatypes and guarded fixpoints. However, as they consider only a strongly-normalizing fragment of the $\lambda$-calculus, establishing correctness becomes significantly simpler. Their implementation takes the form of an abstract machine, whose (5000-line) correctness proof is mechanically checked using a proof assistant. They do not mention how the abstract machine is actually implemented.

Many of the constructions in the present paper are inspired by the first author's work on type-directed partial evaluation [Fil99]. Apart from the obvious differences arising from typed vs. untyped languages, a significant change is also that the TDPE work considered equivalence defined semantically (equality of denotations, under all interpretations of "dynamic" constants), while here we consider syntactic $\beta$ convertibility. Accordingly, the central invariant relation ties denotations to syntactic terms, rather than to denotations in another semantics.

Essentially the same program as in Section 4, but expressed in FreshML, appears in a recent paper by Shinwell et al. [SPG03, Figure 7]. However, the focus there is on a practical application of fresh-name generation, rather than on normalization as such. Indeed, the underlying algorithm (informally attributed to Coquand) is not supported by a formal correctness argument. In our variant, generation of fresh names is handled explicitly: since constructed output terms are never subsequently analyzed by pattern-matching, using a general framework such as FreshML, or higher-order abstract syntax, is probably overkill. However, we anticipate that a different "back end" for output generation could be used, and have deliberately tried to keep the constructions and proofs modular with respect to the term-generation operations. We thus expect that essentially the same arguments - perhaps even a little simplified - could be used to verify correctness of the FreshML variant of the normalizer as well. 


\section{A semantic normalization construction}

\subsection{Syntax and semantics of the untyped $\lambda$-calculus}

Syntax Let $\mathrm{V}=\{\mathrm{x}, \mathrm{y}, \ldots\}$ be a countably infinite set of (object) variables, with $x$ and $v$ ranging over $\mathrm{V}$. The set of $\lambda$-terms $m$ is then the least set $\Lambda$ such that

$$
\begin{aligned}
\Lambda=\{ & \operatorname{VAR}(x) \mid x \in \mathrm{V}\} \cup\left\{\operatorname{LAM}\left(x, m_{0}\right) \mid x \in \mathrm{V}, m_{0} \in \Lambda\right\} \cup \\
& \left\{\operatorname{APP}\left(m_{1}, m_{2}\right) \mid m_{1} \in \Lambda, m_{2} \in \Lambda\right\} .
\end{aligned}
$$

Note that we do not identify $\alpha$-equivalent terms at the level of syntax. The set of free variables of a term, $F V(m)$, is defined in the usual way. For any finite set of variables $\Delta$, we write $\Lambda^{\Delta}$ for the set of $\lambda$-terms over $\Delta$, i.e.,

$$
\Lambda^{\Delta}=\{m \in \Lambda \mid F V(m) \subseteq \Delta\}
$$

Substitutions For technical reasons, we take simultaneous (as opposed to singlevariable), capture-avoiding substitution as the basic concept. Accordingly, we say that a substitution $\theta$ is a finite partial function from variables to terms. We take $F V(\theta)=\bigcup_{x \in \operatorname{dom} \theta} F V(\theta(x))$, and define the action of $\theta$ on a term $m$ in the usual way, by structural induction on $m$ :

$$
\begin{aligned}
\operatorname{VAR}(x)[\theta]= & \begin{cases}\theta(x) & \text { if } x \in \operatorname{dom} \theta \\
\operatorname{VAR}(x) & \text { otherwise }\end{cases} \\
\operatorname{LAM}\left(x, m_{0}\right)[\theta]= & \operatorname{LAM}\left(x^{\prime}, m_{0}\left[\theta\left[x \mapsto \operatorname{VAR}\left(x^{\prime}\right)\right]\right]\right) \\
\quad \text { where } x^{\prime} \notin F V(\theta) \cup\left(F V\left(m_{0}\right) \backslash\{x\}\right) & \operatorname{APP}\left(m_{1}[\theta], m_{2}[\theta]\right)
\end{aligned}
$$

where $f[a \mapsto b]$ is function extension: $f[a \mapsto b]\left(a^{\prime}\right)=b$ if $a^{\prime}=a$, and $f\left(a^{\prime}\right)$ otherwise. To keep the substitution operation deterministic, we assume that the $x^{\prime}$ in the LAMclause is picked as some arbitrary but fixed function of the (finite) set of variables it needs to avoid. As a special case, we use the standard notation $m\left[m^{\prime} / x\right]$ to mean $m\left[\emptyset\left[x \mapsto m^{\prime}\right]\right]$.

Convertibility and normalization We define convertibility between $\lambda$-terms, written $m \leftrightarrow m^{\prime}$, by the axiom schemas for $\alpha$ - and $\beta$-conversion,

$$
\begin{aligned}
\operatorname{LAM}(x, m) & \leftrightarrow \operatorname{LAM}\left(x^{\prime}, m\left[x^{\prime} / x\right]\right) \quad\left(x^{\prime} \notin F V(m) \backslash\{x\}\right) \\
\operatorname{APP}\left(\operatorname{LAM}(x, m), m^{\prime}\right) & \leftrightarrow m\left[m^{\prime} / x\right],
\end{aligned}
$$

together with the standard equivalence and compatibility rules, making $\leftrightarrow$ into a congruence relation on terms.

We further define atomic (also known as neutral) and normal forms, as follows:

$$
\overline{\vdash_{\text {at }} \operatorname{VAR}(x)} \quad \frac{\vdash_{\text {at }} m_{1} \vdash_{\mathrm{nf}} m_{2}}{\vdash_{\text {at }} \operatorname{APP}\left(m_{1}, m_{2}\right)} \quad \frac{\vdash_{\text {at }} m}{\vdash_{\mathrm{nf}} m} \quad \frac{\vdash_{\mathrm{nf}} m_{0}}{\vdash_{\mathrm{nf}} \operatorname{LAM}\left(x, m_{0}\right)} .
$$

For $s \in\{$ nf, at $\}$, we take $\mathcal{N}_{s}=\left\{m \mid \vdash_{s} m\right\}$, i.e., the set of terms that can be shown to be of syntactic form $s$ by a finite number of rule applications.

We then expect a normalization function on terms to satisfy that the output, if any, is in normal form and convertible to the input (soundness); convertible terms either give the same output, or neither one does (identification); and if a term has a normal form at all, the normalization function will return one (completeness). 
Semantics A natural way of defining a denotational model of convertibility is in terms of a reflexive pointed cpo $D$. Reflexivity means that the continuous-function space $[D \rightarrow D]$ is a retract of $D$, i.e., that there exist continuous functions

$$
\phi:[D \rightarrow D] \rightarrow D \quad \text { and } \quad \psi: D \rightarrow[D \rightarrow D]
$$

with $\psi \circ \phi=i d_{[D \rightarrow D]}$. The induced interpretation, $\llbracket m \rrbracket \in[[\mathrm{V} \rightarrow D] \rightarrow D]$, is then:

$$
\begin{aligned}
\llbracket \operatorname{VAR}(x) \rrbracket \rho & =\rho(x) \\
\llbracket \operatorname{LAM}\left(x, m_{0}\right) \rrbracket \rho & =\phi\left(\lambda d^{D} . \llbracket m_{0} \rrbracket \rho[x \mapsto d]\right) \\
\llbracket \operatorname{APP}\left(m_{1}, m_{2}\right) \rrbracket \rho & =\psi\left(\llbracket m_{1} \rrbracket \rho\right)\left(\llbracket m_{2} \rrbracket \rho\right) .
\end{aligned}
$$

Lemma 1 The interpretation has two expectable properties:

a. If $\forall x \in F V(m) . \rho(x)=\rho^{\prime}(x)$, then $\llbracket m \rrbracket \rho=\llbracket m \rrbracket \rho^{\prime}$.

b. Let $\theta=\left[x_{1} \mapsto m_{1}, \ldots, x_{n} \mapsto m_{n}\right]$ be a substitution.

Then $\llbracket m[\theta] \rrbracket \rho=\llbracket m \rrbracket \rho\left[x_{1} \mapsto \llbracket m_{1} \rrbracket \rho, \ldots, x_{n} \mapsto \llbracket m_{n} \rrbracket \rho\right]$.

Proof: Part (a) is a straightforward induction on the structure of $m$. Part (b) follows by induction on the structure of $m$, using part (a) in the LAM-case.

Lemma 2 (model soundness) If $m \leftrightarrow m^{\prime}$ then $\llbracket m \rrbracket=\llbracket m^{\prime} \rrbracket$.

Proof: By induction on the derivation of $m \leftrightarrow m^{\prime}$, using Lemma 1 for $\alpha$ - and $\beta$-conversion, and using that $\psi \circ \phi=i d_{[D \rightarrow D]}$ for $\beta$-conversion.

\subsection{Output-term generation}

We want to account rigorously for the generation of fresh names, and do so in a modular manner. We will therefore construct a pointed cpo $\widehat{\Lambda}$ (dependent on the name generation scheme) with elements denoted by $l$, together with continuous wrapper functions,

$$
\widehat{\mathrm{VAR}}: \mathrm{V} \rightarrow \widehat{\Lambda} \quad \widehat{\mathrm{LAM}}:[\mathrm{V} \rightarrow \widehat{\Lambda}] \rightarrow \widehat{\Lambda} \quad \widehat{\operatorname{APP}}: \widehat{\Lambda} \times \widehat{\Lambda} \rightarrow \widehat{\Lambda},
$$

where, in particular, $\widehat{\mathrm{LAM}}$ provides a fresh name to be used in constructing the body of the $\lambda$-abstraction.

Let $\mathrm{N}$ be a set (discrete cpo) containing at least the natural numbers, with an operation $\cdot+1: \mathrm{N} \rightarrow \mathrm{N}$, agreeing with the successor operation on naturals. Let $\mathrm{G}=\left\{\mathrm{g}_{0}, \mathrm{~g}_{1}, \ldots\right\}$ be a countably infinite subset of $\mathrm{V}$, such that $\mathrm{g}_{i}=\mathrm{g}_{j}$ implies $i=j$, and let gen $: \mathrm{N} \rightarrow \mathrm{V}$ be such that $\operatorname{gen}(n)=\mathrm{g}_{n}$ when $n \in \omega$.

We write $\lfloor\cdot\rfloor$ for the inclusion from $A$ to $A_{\perp}$; and for $f: A \rightarrow B$ with $B$ pointed, we write $\star \star f$ for $f$ 's strict extension to $A_{\perp}$, i.e., $\perp \star f=\perp_{B}$ and $\lfloor a\rfloor \star f=f(a)$. (As is conventional in functional-programming syntax, function application by juxtaposition binds tighter than all explicit infix operators, including $\star$.)

Definition 1 We take $\widehat{\Lambda}=\left[\mathrm{N} \rightarrow \Lambda_{\perp}\right]$ and define wrapper functions for constructing $\lambda$-terms using de Bruijn-level (not-index!) naming as follows:

$$
\begin{aligned}
\widehat{\operatorname{VAR}}(v) & =\lambda n^{\mathrm{N}} \cdot\lfloor\operatorname{VAR}(v)\rfloor \\
\widehat{\operatorname{LAM}}(f) & =\lambda n^{\mathrm{N}} \cdot f(\operatorname{gen}(n))(n+1) \star \lambda m_{0}^{\Lambda} \cdot\left\lfloor\operatorname{LAM}\left(\operatorname{gen}(n), m_{0}\right)\right\rfloor \\
\widehat{\operatorname{APP}}\left(l_{1}, l_{2}\right) & =\lambda n^{\mathrm{N}} \cdot l_{1} n \star \lambda m_{1}^{\Lambda} \cdot l_{2} n \star \lambda m_{2}^{\Lambda} \cdot\left\lfloor\operatorname{APP}\left(m_{1}, m_{2}\right)\right\rfloor .
\end{aligned}
$$


Note 1 If we took freshness as a primitive concept, like in FreshML, we could simply use $\widehat{\Lambda}=\Lambda_{\perp} ; \widehat{\operatorname{VAR}}(v)=\lfloor\operatorname{VAR}(v)\rfloor ; \widehat{\operatorname{LAM}}(f)=f x \star \lambda m_{0} \cdot\left\lfloor\operatorname{LAM}\left(x, m_{0}\right)\right\rfloor$, with $x$ fresh for $f$; and $\widehat{\operatorname{APP}}\left(l_{1}, l_{2}\right)=l_{1} \star \lambda m_{1} \cdot l_{2} \star \lambda m_{2} \cdot\left\lfloor\operatorname{APP}\left(m_{1}, m_{2}\right)\right\rfloor$.

\subsection{A residualizing model}

From standard domain-theoretic results (e.g., Pitts [Pit93]), we know that there exists a pointed cpo $D_{\mathrm{r}}$, together with an isomorphism

$$
i_{D}: D_{\mathrm{r}} \cong\left(\widehat{\Lambda}+\left[D_{\mathrm{r}} \rightarrow D_{\mathrm{r}}\right]\right)_{\perp} .
$$

We write

$$
\operatorname{tm}(l)=i_{D}^{-1}\left(\left\lfloor i n_{1}(l)\right\rfloor\right) \quad \text { fun }(f)=i_{D}^{-1}\left(\left\lfloor i n_{2}(f)\right\rfloor\right) \quad \perp_{D_{\mathrm{r}}}=i_{D}^{-1}(\perp) .
$$

Then any element of $D_{\mathrm{r}}$ can be uniquely written as one of $\operatorname{tm}(l)$, fun $(f)$, or $\perp_{D_{\mathrm{r}}}$.

Moreover, the standard domain-theoretic solution is in fact a so-called minimal invariant [Pit93], which we will exploit in the correctness proof. (In the specific case of $D_{\mathrm{r}}$, the minimal-invariant condition says that the following "copy function" $e: D_{\mathrm{r}} \rightarrow D_{\mathrm{r}}$, recursively defined in the least-fixed-point sense,

$$
e(d)=\text { case } d \text { of }\left\{\begin{aligned}
\operatorname{tm}(l) & \rightarrow \operatorname{tm}(l) \\
\operatorname{fun}(f) & \rightarrow \text { fun }(e \circ f \circ e) \\
\perp_{D_{\mathrm{r}}} & \rightarrow \perp_{D_{\mathrm{r}}}
\end{aligned}\right.
$$

is in fact the identity function on $D_{\mathrm{r}}$.)

We can now define the reification function, $\downarrow: D_{\mathrm{r}} \rightarrow \widehat{\Lambda}$, and the reflection function, $\uparrow: \widehat{\Lambda} \rightarrow D_{\mathrm{r}}$, as follows:

$$
\begin{aligned}
& \downarrow d=\text { case } d \text { of }\left\{\begin{aligned}
\operatorname{tm}(l) & \rightarrow l \\
\operatorname{fun}(f) & \rightarrow \widehat{\operatorname{LAM}}\left(\lambda x^{\mathrm{V}} \cdot \downarrow(f(\uparrow(\widehat{\operatorname{VAR}}(x))))\right) \\
\perp_{D_{\mathrm{r}}} & \rightarrow \perp_{\widehat{\Lambda}}
\end{aligned}\right\} \operatorname{tm}(l),
\end{aligned}
$$

where the recursive definition of $\downarrow$ is again interpreted as the least fixed point. Using these, we construct appropriate functions $\phi_{\mathrm{r}}:\left[D_{\mathrm{r}} \rightarrow D_{\mathrm{r}}\right] \rightarrow D_{\mathrm{r}}$ and $\psi_{\mathrm{r}}: D_{\mathrm{r}} \rightarrow\left[D_{\mathrm{r}} \rightarrow D_{\mathrm{r}}\right]$ :

$$
\begin{aligned}
\phi_{\mathrm{r}}(f) & =\operatorname{fun}(f) \\
\psi_{\mathrm{r}}(d) & =\text { case } d \text { of }\left\{\begin{aligned}
\operatorname{tm}(l) & \rightarrow \lambda d^{\prime D_{\mathrm{r}}} \cdot \uparrow \widehat{\operatorname{APP}}\left(l, \downarrow d^{\prime}\right) \\
\operatorname{fun}(f) & \rightarrow f \\
\perp_{D_{\mathrm{r}}} & \rightarrow \perp_{\left[D_{\mathrm{r}} \rightarrow D_{\mathrm{r}}\right]} .
\end{aligned}\right.
\end{aligned}
$$

Clearly, we have that $\psi_{\mathrm{r}} \circ \phi_{\mathrm{r}}=i d_{\left[D_{\mathrm{r}} \rightarrow D_{\mathrm{r}}\right]}$, since $i_{D}$ was an isomorphism. The induced interpretation is denoted by $\llbracket \cdot \rrbracket_{\mathrm{r}}$. We can now define a putative normalization function:

Definition 2 For any $\Delta$, let $\sharp \Delta=\max \left(\left\{n+1 \mid \mathrm{g}_{n} \in \Delta\right\} \cup\{0\}\right)$ (i.e., the least $n$ such that $\forall n^{\prime} \geq n$. $\left.\mathrm{g}_{n^{\prime}} \notin \Delta\right)$. We then define the function $\operatorname{norm}_{\Delta}: \Lambda^{\Delta} \rightarrow \Lambda_{\perp}$ by

$$
\operatorname{norm}_{\Delta}(m)=\downarrow\left(\llbracket m \rrbracket_{\mathrm{r}}\left(\lambda x^{\mathrm{V}} \cdot \uparrow(\widehat{\operatorname{VAR}}(x))\right)\right) \sharp \Delta .
$$

We also define the general function norm : $\Lambda \rightarrow \Lambda_{\perp}$ like above, but with $\sharp \Delta$ replaced by 0 . Then for any $\Delta$ such that $\Delta \cap \mathrm{G}=\emptyset$, and $m \in \Lambda^{\Delta}, \operatorname{norm}(m)=\operatorname{norm}_{\Delta}(m)$. 


\section{Correctness of the construction}

\subsection{Correctness of the wrappers}

We first define what it means for an element of $\widehat{\Lambda}$ to represent a $\lambda$-term with some additional properties:

Definition 3 For $l \in \widehat{\Lambda}, \Delta \subseteq$ fin $\mathrm{V}, s \in\{$ at, nf $\}$, and $m \in \Lambda^{\Delta}$, we define the representation relation $\lesssim b y$

$$
l \lesssim_{s}^{\Delta} m \text { iff } \forall n \geq \sharp \Delta . l n=\perp \vee \exists m^{\prime} \in \Lambda^{\Delta} . l n=\left\lfloor m^{\prime}\right\rfloor \wedge m^{\prime} \leftrightarrow m \wedge m^{\prime} \in \mathcal{N}_{s} .
$$

That is, as long as we avoid clashes with generated bound-variable names, any concrete term generated from $l$ has only free variables in $\Delta$, is convertible to $m$, and is of syntactic form $s$. Note, however, that we only capture a notion of partial correctness here: if $l$ does not generate a term at all, the conditions are vacuously satisfied.

Lemma 3 For fixed $\Delta, s$, and $m$, the predicate $P=\left\{l \mid l \lesssim_{s}^{\Delta} m\right\}$ is pointed (i.e., $\perp_{\widehat{\Lambda}} \in P$ ) and inclusive (i.e., closed under limits of $\omega$-chains).

Proof: Straightforward, noting that $\lesssim$ is expressed as an intersection of inverse images by a continuous function (application to $n$ ) of a (necessarily inclusive) predicate on the flat domain $\Lambda_{\perp}$.

Lemma 4 The representation relation is closed under weakening and conversion:

a. If $l \lesssim_{s}^{\Delta} m$ and $\Delta \subseteq \Delta^{\prime}$, then also $l \lesssim_{s}^{\Delta^{\prime}} m$.

b. If $l \lesssim_{s}^{\Delta} m$ and $m^{\prime} \in \Lambda^{\Delta}$ with $m \leftrightarrow m^{\prime}$, then also $l \lesssim_{s}^{\Delta} m^{\prime}$.

Proof: Both parts are immediate from the definition. For part (a), assume $l \lesssim_{s}^{\Delta} m$ and $\Delta \subseteq \Delta^{\prime}$, and consider an $n \geq \sharp \Delta^{\prime}$. Then also $n \geq \sharp \Delta$, and thus, by the assumption on $l$, if $l n \neq \perp$, there exists an $m^{\prime} \in \Lambda^{\Delta} \subseteq \Lambda^{\Delta^{\prime}}$, satisfying the conditions in $l \lesssim s m$.

For part (b), assume $l \lesssim_{s}^{\Delta} m$, and let $m^{\prime} \in \Lambda^{\Delta}$ with $m \leftrightarrow m^{\prime}$ be given. Then, when $n \geq \sharp \Delta$ and $l n \neq \perp$, there exists an $m^{\prime \prime} \in \Lambda^{\Delta}$ such that $l n=\left\lfloor m^{\prime \prime}\right\rfloor, m^{\prime \prime} \leftrightarrow m$, and $m^{\prime \prime} \in \mathcal{N}_{s}$. By transitivity of $\leftrightarrow$, we must also have $m^{\prime \prime} \leftrightarrow m^{\prime}$, so $l \lesssim_{s} m^{\prime}$ as required.

Lemma 5 Representations of terms behave much like the terms themselves:

a. If $v \in \Delta$, then $\widehat{\operatorname{VAR}}(v) \lesssim{ }_{\text {at }} \operatorname{VAR}(v)$.

b. If $l_{1} \lesssim_{\text {at }}^{\Delta} m_{1}$ and $l_{2} \lesssim_{\mathrm{nf}}^{\Delta} m_{2}$, then $\widehat{\operatorname{APP}}\left(l_{1}, l_{2}\right) \lesssim_{\text {at }}^{\Delta} \operatorname{APP}\left(m_{1}, m_{2}\right)$.

c. If $l \lesssim \Delta$ at $m$, then also $l \lesssim_{\mathrm{nf}}^{\Delta} m$.

d. Let $f \in[\mathrm{V} \rightarrow \widehat{\Lambda}]$ and $m \in \Lambda^{\Delta \cup\{x\}}$. If $\forall v \notin \Delta$.fv $\lesssim_{\mathrm{nf}}^{\Delta \cup\{v\}} m[\operatorname{VAR}(v) / x]$, then $\widehat{\operatorname{LAM}}(f) \lesssim_{\mathrm{nf}}^{\Delta} \operatorname{LAM}(x, m)$. 
Proof: Parts (a), (b), and (c) are straightforward, where (b) uses that convertibility is a congruence wrt. APP. We will now prove $(\mathrm{d})$.

Let $f, x$, and $m$, satisfy the condition of the lemma, and let $n \geq \sharp \Delta$ and $m^{\prime}$ with $\widehat{\mathrm{LAM}}(f) n=\left\lfloor m^{\prime}\right\rfloor$ be given; we must show that $m^{\prime} \in \Lambda^{\Delta}, m^{\prime} \leftrightarrow \operatorname{LAM}(x, m)$, and $m^{\prime} \in \mathcal{N}_{\text {nf }}$.

From the definition of $\widehat{\operatorname{LAM}}(f)$, we must have that, for some $m_{0}, f \mathrm{~g}_{n}(n+1)=$ $\left\lfloor m_{0}\right\rfloor$ and $m^{\prime}=\operatorname{LAM}\left(\mathrm{g}_{n}, m_{0}\right)$. By definition of $\sharp, \mathrm{g}_{n} \notin \Delta$, so by assumption on $f$, $f \mathrm{~g}_{n} \lesssim_{\mathrm{nf}}^{\Delta \cup\left\{\mathrm{g}_{n}\right\}} m\left[\operatorname{VAR}\left(\mathrm{g}_{n}\right) / x\right]$. Further, since $n+1 \geq \sharp\left(\Delta \cup\left\{\mathrm{g}_{n}\right\}\right)$, the definition of gives us that $m_{0} \in \Lambda^{\Delta \cup\left\{\mathrm{g}_{n}\right\}}, m_{0} \leftrightarrow m\left[\operatorname{VAR}\left(\mathrm{g}_{n}\right) / x\right]$, and $m_{0} \in \mathcal{N}_{\text {nf }}$, . But then clearly $\operatorname{LAM}\left(\mathrm{g}_{n}, m_{0}\right) \in \Lambda^{\Delta}, \operatorname{LAM}\left(\mathrm{g}_{n}, m_{0}\right) \in \mathcal{N}_{\mathrm{nf}}$, and

$$
\operatorname{LAM}\left(\mathrm{g}_{n}, m_{0}\right) \leftrightarrow \operatorname{LAM}\left(\mathrm{g}_{n}, m\left[\operatorname{VAR}\left(\mathrm{g}_{n}\right) / x\right]\right) \leftrightarrow \operatorname{LAM}(x, m),
$$

where the first conversion is by congruence wrt. LAM and the second is a valid $\alpha$ conversion, since $\mathrm{g}_{n} \notin \Delta$ ensures that $\mathrm{g}_{n} \notin F V(m) \backslash\{x\}$.

The constructions and results in the next section rely only on those properties of the wrappers from Definition 1 and the relation $\lesssim$ from Definition 3 that were established in Lemmas 3-5, not on the definitions themselves.

\subsection{Adequacy of the residualizing model}

To construct the central relation between terms and their residualizing denotations, we first state an abstract version of a result due to Pitts [Pit93]:

Theorem 1 (existence of invariant relations) Let $A$ be a cpo, and let $i: D \cong$ $(A+[D \rightarrow D])_{\perp}$ be a minimal invariant. Let $T$ be a set, and let predicates $P_{1} \subseteq A \times T$, $P_{2} \subseteq T$, and $P_{3} \subseteq T \times T \times T$ be given, such that $\left\{a \mid P_{1}(a, t)\right\}$ is inclusive for every $t \in T$. Then there exists a relation $\triangleleft \subseteq D \times T$, with $\{d \mid d \triangleleft t\}$ inclusive for every $t \in T$, such that, for all $d \in D$ and $t \in T$,

$$
d \triangleleft t \text { iff }\left(\begin{array}{l}
d=\perp_{D} \vee \\
\exists a . d=i^{-1}\left(\left\lfloor i n_{1}(a)\right\rfloor\right) \wedge P_{1}(a, t) \vee \\
\exists f . d=i^{-1}\left(\left\lfloor i n_{2}(f)\right\rfloor\right) \wedge P_{2}(t) \wedge \\
\forall d^{\prime} \in D ; t^{\prime}, t^{\prime \prime} \in T . P_{3}\left(t, t^{\prime}, t^{\prime \prime}\right) \wedge d^{\prime} \triangleleft t^{\prime} \Rightarrow f\left(d^{\prime}\right) \triangleleft t^{\prime \prime}
\end{array}\right) .
$$

Proof: See Appendix A

We can recover Pitts's original result as follows. Let $\Lambda_{\mathbb{Z}}$ be an extension of $\Lambda$ with PCF-style integer arithmetic, and let $\Downarrow$ be the usual big-step, call-by-name evaluation relation on $\Lambda_{\mathbb{Z}}^{\emptyset}$. We then take $A$ as $\mathbb{Z}, T$ as $\Lambda_{\mathbb{Z}}^{\emptyset}, P_{1}(n, m)$ as $m \Downarrow \underline{n}, P_{2}(m)$ as $\exists x, m_{0} . m \Downarrow \operatorname{LAM}\left(x, m_{0}\right)$, and $P_{3}\left(m, m^{\prime}, m^{\prime \prime}\right)$ as $\forall x, m_{0} \cdot m \Downarrow \operatorname{LAM}\left(x, m_{0}\right) \Rightarrow m^{\prime \prime}=$ $m_{0}\left[m^{\prime} / x\right]$. Note that, by determinacy of $\Downarrow$, the $x$ and $m_{0}$ are uniquely determined when they exist, so $P_{2}$ and $P_{3}$ would naturally be joined into a single condition.

The computational-adequacy proof for evaluation then shows, by a straightforward structural induction on $m$, that if $\rho: \mathrm{V} \rightarrow D$ and $\theta: \mathrm{V} \rightarrow \Lambda_{\mathbb{Z}}^{\emptyset}$ are such that $\forall x \in$ $F V(m) . \rho(x) \triangleleft \theta(x)$, then $\llbracket m \rrbracket \rho \triangleleft m[\theta]$. For the special case when $m$ is itself a closed term, we then immediately read off that if $\llbracket m \rrbracket \emptyset \neq \perp_{D}$ then $m$ must evaluate to a value. 
When generalizing to normalization, there are two complications. First, we consider symmetric equivalence, not directed evaluation, so the relation $d \triangleleft-$ must be closed under arbitrary $\beta$-conversions, not just head- $\beta$-expansions as before. Second, since we also normalize under lambdas, we must in general consider substitutions that replace variables with open terms. Accordingly, we replace the fixed set of closed terms, $\Lambda^{\emptyset}$, with a Kripke-style family of term sets, indexed by their allowed free variables, $\Lambda^{\Delta}$. Somewhat surprisingly, Pitts's result - although in the generalized formulation - accounts directly for these adaptations:

Lemma 6 There exists a relation $\lesssim$ such that for all $\Delta, d \in D_{\mathrm{r}}$ and $m \in \Lambda^{\Delta}$,

$$
d \lesssim^{\Delta} m \text { iff }\left(\begin{array}{c}
d=\perp_{D_{\mathrm{r}}} \vee \\
\exists l . d=\operatorname{tm}(l) \wedge l \lesssim \Delta \text { at } m \vee \\
\exists f . d=\operatorname{fun}(f) \wedge\left(\exists x \in \mathrm{V}, m_{0} \in \Lambda^{\Delta \cup\{x\}} \cdot \operatorname{LAM}\left(x, m_{0}\right) \leftrightarrow m\right) \\
\wedge \forall \Delta^{\prime} \supseteq \Delta, d^{\prime} \in D_{\mathrm{r}}, m^{\prime} \in \Lambda^{\Delta^{\prime}}, m_{1} \in \Lambda^{\Delta^{\prime}} . \\
m \leftrightarrow m_{1} \wedge d^{\prime} \lesssim^{\prime} m^{\prime} \Rightarrow f\left(d^{\prime}\right) \lesssim^{\Delta^{\prime}} \operatorname{APP}\left(m_{1}, m^{\prime}\right)
\end{array}\right) .
$$

Proof: By Theorem 1, taking $A=\widehat{\Lambda}$ and $T=\left\{(\Delta, m) \mid \Delta \subseteq_{\text {fin }} \mathrm{V} \wedge m \in \Lambda^{\Delta}\right\}$, with the predicates chosen as

$$
\begin{aligned}
P_{1}= & \left\{(l,(\Delta, m)) \mid l \lesssim_{\text {at }}^{\Delta}\right\} \\
P_{2}= & \left\{(\Delta, m) \mid \exists x \in \mathrm{V}, m_{0} \in \Lambda^{\Delta \cup\{x\}} \cdot \operatorname{LAM}\left(x, m_{0}\right) \leftrightarrow m\right\} \\
P_{3}= & \left\{\left((\Delta, m),\left(\Delta^{\prime}, m^{\prime}\right),\left(\Delta^{\prime \prime}, m^{\prime \prime}\right)\right) \mid\right. \\
& \left.\Delta \subseteq \Delta^{\prime}=\Delta^{\prime \prime} \wedge \exists m_{1} \in \Lambda^{\Delta^{\prime}} . m \leftrightarrow m_{1} \wedge m^{\prime \prime}=\operatorname{APP}\left(m_{1}, m^{\prime}\right)\right\}
\end{aligned}
$$

using the equivalence $[\forall x .(\exists y \cdot P(x, y)) \Rightarrow Q(x)] \Leftrightarrow[\forall x \cdot \forall y \cdot P(x, y) \Rightarrow Q(x)] . \quad P_{1}$ is inclusive in its first argument by Lemma 3. We write $d \lesssim^{\Delta} m$ instead of $d \triangleleft(\Delta, m)$.

Note how, in the function case, we require that $f$ and $m$ can also be meaningfully applied to arguments from later worlds $\Delta^{\prime}$, much like in a conventional, type-indexed Kripke logical relation [Mit96, p.590].

Lemma 7 The relation $\lesssim$ shares two key properties with :

a. If $d \lesssim^{\Delta} m$ and $\Delta \subseteq \Delta^{\prime}$, then also $d \lesssim^{\prime} m$.

b. If $d \lesssim^{\Delta} m$ and $m^{\prime} \in \Lambda^{\Delta}$ with $m \leftrightarrow m^{\prime}$, then also $d \lesssim^{\Delta} m^{\prime}$.

Proof: We proceed according to the cases for $d \lesssim^{\Delta} m$ in Lemma 6 :

Case $d=\perp_{D_{\mathrm{r}}}$ : Both parts are immediate.

Case $d=\operatorname{tm}(l)$ : Both parts follow directly from the corresponding parts of Lemma 4 , taking $s=$ at.

Case $d=$ fun $(f)$ : For (a), if $m_{0} \in \Lambda^{\Delta \cup\{x\}}$, then also $m_{0} \in \Lambda^{\Delta^{\prime} \cup\{x\}}$. Likewise, any $\Delta^{\prime \prime}$ with $\Delta^{\prime \prime} \supseteq \Delta^{\prime}$ in the universal quantification also satisfies $\Delta^{\prime \prime} \supseteq \Delta$.

For (b), any $m_{0}$ satisfying $\operatorname{LAM}\left(x, m_{0}\right) \leftrightarrow m$ also satisfies $\operatorname{LAM}\left(x, m_{0}\right) \leftrightarrow m^{\prime}$ by transitivity. Similarly, the terms $m_{1}$ satisfying $m \leftrightarrow m_{1}$ are the same as those that satisfy $m^{\prime} \leftrightarrow m_{1}$. 
The following two lemmas will combine to establish adequacy of our semantics:

Lemma 8 For all $l \in \widehat{\Lambda}, d \in D_{\mathrm{r}}$, and $m \in \Lambda^{\Delta}$,

a. If $l \lesssim \Delta$ at $m$, then $\uparrow l \lesssim^{\Delta} m$.

b. If $d \lesssim^{\Delta} m$, then $\downarrow d \lesssim_{\mathrm{nf}}^{\Delta} m$.

Proof: Part (a) follows immediately from Lemma $6(\Leftarrow)$ and the definition of $\uparrow$.

For part (b), recall that reification was conceptually defined in terms of the continuous function $\Phi:\left[D_{\mathrm{r}} \rightarrow \widehat{\Lambda}\right] \rightarrow\left[D_{\mathrm{r}} \rightarrow \widehat{\Lambda}\right]$,

$$
\Phi(\varphi)=\lambda d^{D_{\mathrm{r}}} \text {. case } d \text { of }\left\{\begin{aligned}
\operatorname{tm}(l) & \rightarrow l \\
\operatorname{fun}(f) & \rightarrow \widehat{\operatorname{LAM}}\left(\lambda x^{\mathrm{V}} \cdot \varphi(f(\uparrow(\widehat{\operatorname{VAR}}(x))))\right) \\
\perp_{D_{\mathrm{r}}} & \rightarrow \perp_{\widehat{\Lambda}}
\end{aligned}\right.
$$

with $\downarrow=\operatorname{fix}(\Phi)$. Consider therefore the predicate

$$
R=\left\{\varphi \in\left[D_{\mathrm{r}} \rightarrow \widehat{\Lambda}\right] \mid \forall d, \Delta, m \in \Lambda^{\Delta} . d \lesssim^{\Delta} m \Rightarrow \varphi(d) \lesssim \mathrm{nf}^{\Delta} m\right\} .
$$

It is straightforward to verify that $R$ is pointed and inclusive, using the corresponding properties of $\lesssim$ (Lemma 3 ). To show that $\operatorname{fix}(\Phi) \in R$ by fixed-point induction, it therefore suffices to show that for all $\varphi \in R, \Phi(\varphi) \in R$.

Accordingly, assume that $\varphi \in R$ and $d \lesssim^{\Delta} m$; we aim to prove that $\Phi(\varphi)(d) \lesssim_{\text {nf }}^{\Delta} m$. We divide the argument into cases over $d$ :

Case $d=\perp_{D_{\mathrm{r}}}$ : Then $\Phi(\varphi)(d)=\perp_{\widehat{\Lambda}}$, and thus $\perp_{\widehat{\Lambda}} \lesssim_{\text {nf }}^{\Delta} m$, by Lemma 3 .

Case $d=\operatorname{tm}(l)$ : Then $\Phi(\varphi)(d)=l$, and by Lemma $6(\Rightarrow)$ and Lemma $5(\mathrm{c}), l \lesssim_{\mathrm{nf}} m$.

Case $d=\operatorname{fun}(f)$ : Then $\Phi(\varphi)(d)=\widehat{\mathrm{LAM}}\left(\lambda x^{\mathrm{V}} \cdot \varphi(f(\uparrow(\widehat{\operatorname{VAR}}(x))))\right)$. Let $v \notin \Delta$ be arbitrary. By Lemma $5(\mathrm{a}), \widehat{\operatorname{VAR}}(v) \gtrsim_{\text {at }}^{\Delta \cup\{v\}} \operatorname{VAR}(v)$, and so by part (a) above,

$$
\uparrow(\widehat{\operatorname{VAR}}(v)) \lesssim^{\Delta \cup\{v\}} \operatorname{VAR}(v) .
$$

By assumption on $m$ and Lemma $6(\Rightarrow)$, there exist $x$ and $m_{0} \in \Lambda^{\Delta \cup\{x\}}$ such that $\operatorname{LAM}\left(x, m_{0}\right) \leftrightarrow m$.

Take $\Delta^{\prime}=\Delta \cup\{v\}, d^{\prime}=\uparrow(\widehat{\operatorname{VAR}}(v)), m^{\prime}=\operatorname{VAR}(v)$, and $m_{1}=\operatorname{LAM}\left(x, m_{0}\right)$. By assumption on $f$, we then get that

$$
f(\uparrow(\widehat{\operatorname{VAR}}(v))) \lesssim^{\Delta \cup\{v\}} \operatorname{APP}\left(\operatorname{LAM}\left(x, m_{0}\right), \operatorname{VAR}(v)\right) .
$$

Since $\operatorname{APP}\left(\operatorname{LAM}\left(x, m_{0}\right), \operatorname{VAR}(v)\right) \leftrightarrow m_{0}[\operatorname{VAR}(v) / x]$, and $\lesssim$ is closed under conversion (Lemma 7(b)), we also have

$$
f(\uparrow(\widehat{\operatorname{VAR}}(v))) \lesssim^{\Delta \cup\{v\}} m_{0}[\operatorname{VAR}(v) / x] .
$$

Hence, by assumption on $\varphi$,

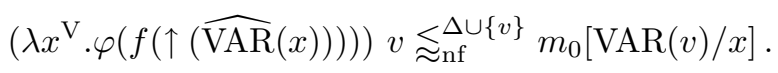


And thus, by Lemma 5(d),

$$
\widehat{\operatorname{LAM}}\left(\lambda x^{\mathrm{V}} \cdot \varphi(f(\uparrow(\widehat{\operatorname{VAR}}(x))))\right) \lesssim_{\mathrm{nf}}^{\Delta} \operatorname{LAM}\left(x, m_{0}\right) .
$$

Finally, since $\lesssim$ is closed under conversion (Lemma $4(\mathrm{~b})$ ), we get $\Phi(\varphi)(d) \lesssim_{\text {nf }}^{\Delta} m$, as required.

Lemma 9 Let $m \in \Lambda^{\Gamma}$, and for all $x \in \Gamma$, let $\theta(x) \in \Lambda^{\Delta}$ (in particular, $\Gamma \subseteq \operatorname{dom} \theta$ ). If $\forall x \in \Gamma$. $\rho(x) \lesssim^{\Delta} \theta(x)$ then $\llbracket m \rrbracket_{\mathrm{r}} \rho \lesssim^{\Delta} m[\theta]$.

Proof: By structural induction on $m$.

Case $m=\operatorname{VAR}(x)$ : This follows immediately from the assumption on $\rho$ and $\theta$, since $\llbracket \operatorname{VAR}(x) \rrbracket_{\mathrm{r}} \rho=\rho(x)$.

Case $m=\operatorname{LAM}\left(x, m_{0}\right)$ : Take $f=\lambda d \cdot \llbracket m_{0} \rrbracket_{\mathrm{r}} \rho[x \mapsto d]$. Then $i_{D}\left(\llbracket m \rrbracket_{\mathrm{r}} \rho\right)=\left\lfloor i n_{2}(f)\right\rfloor$, so to use Lemma $6(\Leftarrow)$, we must establish that $f$ and $m[\theta]$ satisfy the requirements for the third alternative. First, from the definition of substitution, we get that $\operatorname{LAM}\left(x, m_{0}\right)[\theta]=\operatorname{LAM}\left(x^{\prime}, m_{0}^{\prime}\right)$ for some $x^{\prime}$ and $m_{0}^{\prime}=m_{0}\left[\theta\left[x \mapsto \operatorname{VAR}\left(x^{\prime}\right)\right]\right]$. Clearly $m_{0}^{\prime} \in \Lambda^{\Delta \cup\left\{x^{\prime}\right\}}$, and $\operatorname{LAM}\left(x^{\prime}, m_{0}^{\prime}\right) \leftrightarrow m[\theta]$ by reflexivity of $\leftrightarrow$.

Second, let $\Delta^{\prime} \supseteq \Delta, d^{\prime}, m_{1} \in \Lambda^{\Delta^{\prime}}$ and $m^{\prime} \in \Lambda^{\Delta^{\prime}}$ be given, with $m[\theta] \leftrightarrow m_{1}$ and $d^{\prime} \lesssim^{\Delta^{\prime}} m^{\prime}$; we must show that $f\left(d^{\prime}\right) \lesssim^{\Delta^{\prime}} \operatorname{APP}\left(m_{1}, m^{\prime}\right)$. Take $\rho^{\prime}=\rho\left[x \mapsto d^{\prime}\right]$ and $\theta^{\prime} \stackrel{=}{=} \theta\left[x \mapsto m^{\prime}\right]$. Using the assumption on $d^{\prime}$ and $m^{\prime}$ for $x$, and monotonicity of $\lesssim$ (Lemma 7(a)) for the remaining variables in $\Gamma$, we get that for all $x^{\prime \prime} \in \Gamma \cup\{x\}$, $\rho^{\prime}\left(x^{\prime \prime}\right) \lesssim^{\Delta^{\prime}} \theta^{\prime}\left(x^{\prime \prime}\right)$. Hence, by IH on $m_{0}, f\left(d^{\prime}\right)=\llbracket m_{0} \rrbracket_{\mathrm{r}} \rho^{\prime} \lesssim_{\Delta^{\prime}} m_{0}\left[\theta^{\prime}\right]$. And finally, since

$$
\begin{aligned}
& m_{0}\left[\theta^{\prime}\right] \\
& \leftrightarrow \operatorname{APP}\left(\operatorname{LAM}\left(x, m_{0}\right), \operatorname{VAR}(x)\right)\left[\theta^{\prime}\right]=\operatorname{APP}\left(\operatorname{LAM}\left(x, m_{0}\right)\left[\theta^{\prime}\right], \operatorname{VAR}(x)\left[\theta^{\prime}\right]\right) \\
& \leftrightarrow \operatorname{APP}\left(\operatorname{LAM}\left(x, m_{0}\right)[\theta], m^{\prime}\right)=\operatorname{APP}\left(m[\theta], m^{\prime}\right) \\
& \leftrightarrow \operatorname{APP}\left(m_{1}, m^{\prime}\right),
\end{aligned}
$$

and $\lesssim$ is closed under conversion (Lemma $7(\mathrm{~b}))$, we get $f\left(d^{\prime}\right) \lesssim^{\Delta^{\prime}} \operatorname{APP}\left(m_{1}, m^{\prime}\right)$, as required.

Case $m=\operatorname{APP}\left(m_{1}, m_{2}\right)$ : Here, $\llbracket \operatorname{APP}\left(m_{1}, m_{2}\right) \rrbracket_{\mathrm{r}} \rho=\psi_{\mathrm{r}}\left(\llbracket m_{1} \rrbracket_{\mathrm{r}} \rho\right)\left(\llbracket m_{2} \rrbracket_{\mathrm{r}} \rho\right)$. We divide the argument into subcases over $\llbracket m_{1} \rrbracket_{\mathrm{r}} \rho$ :

Case $\llbracket m_{1} \rrbracket_{\mathrm{r}} \rho=\perp_{D_{\mathrm{r}}}$ : Then $\psi_{\mathrm{r}}\left(\llbracket m_{1} \rrbracket_{\mathrm{r}} \rho\right)\left(\llbracket m_{2} \rrbracket_{\mathrm{r}} \rho\right)=\perp \lesssim^{\Delta} \operatorname{APP}\left(m_{1}, m_{2}\right)[\theta]$.

Case $\llbracket m_{1} \rrbracket_{\mathrm{r}} \rho=\operatorname{tm}(l)$ : Then $\psi_{\mathrm{r}}\left(\llbracket m_{1} \rrbracket_{\mathrm{r}} \rho\right)\left(\llbracket m_{2} \rrbracket_{\mathrm{r}} \rho\right)=\uparrow\left(\widehat{\operatorname{APP}}\left(l, \downarrow\left(\llbracket m_{2} \rrbracket_{\mathrm{r}} \rho\right)\right)\right)$. By IH on $m_{1}$ and Lemma $6(\Rightarrow), l \lesssim \Delta$ at $m_{1}[\theta]$, and by IH on $m_{2}$ and Lemma $8(\mathrm{~b})$, $\downarrow\left(\llbracket m_{2} \rrbracket_{\mathrm{r}} \rho\right) \lesssim_{\text {nf }}^{\Delta} m_{2}[\theta]$. Hence by Lemma $5(\mathrm{~b})$,

$$
\widehat{\operatorname{APP}}\left(l, \downarrow\left(\llbracket m_{2} \rrbracket_{\mathrm{r}} \rho\right)\right) \lesssim_{\mathrm{at}}^{\Delta} \operatorname{APP}\left(m_{1}[\theta], m_{2}[\theta]\right)=\operatorname{APP}\left(m_{1}, m_{2}\right)[\theta]=m[\theta] .
$$

And thus, by Lemma $8(\mathrm{a}), \uparrow\left(\widehat{\operatorname{APP}}\left(l, \downarrow \llbracket m_{2} \rrbracket_{\mathrm{r}} \rho\right)\right) \lesssim^{\Delta} m[\theta]$.

Case $\llbracket m_{1} \rrbracket_{\mathrm{r}} \rho=$ fun $(f)$ : Then $\psi_{\mathrm{r}}\left(\llbracket m_{1} \rrbracket_{\mathrm{r}} \rho\right)\left(\llbracket m_{2} \rrbracket_{\mathrm{r}} \rho\right)=f\left(\llbracket m_{2} \rrbracket_{\mathrm{r}} \rho\right)$. By IH on $m_{1}$ and Lemma $6(\Rightarrow)$, we have, in particular, that if $d^{\prime} \lesssim^{\Delta} m^{\prime}$ then $f\left(d^{\prime}\right) \lesssim^{\Delta}$ $\operatorname{APP}\left(m_{1}[\theta], m^{\prime}\right)$. Take $d^{\prime}=\llbracket m_{2} \rrbracket_{\mathrm{r}} \rho$ and $m^{\prime}=m_{2}[\theta]$. Then, using IH on $m_{2}$, $f\left(\llbracket m_{2} \rrbracket_{\mathrm{r}} \rho\right) \lesssim^{\Delta} \operatorname{APP}\left(m_{1}[\theta], m_{2}[\theta]\right)=m[\theta]$. 


\subsection{Correctness of the normalization function}

For showing completeness of the normalizer, we first establish that, for a term already in normal form, reifying its residualizing denotation gives an always-defined term generator.

Definition 4 For any $l \in \widehat{\Lambda}$, we define the uniform definedness predicate $\operatorname{def}(l)$ by $\operatorname{def}(l) \Leftrightarrow \forall n \in \omega . l n \neq \perp$.

Lemma 10 The wrapper functions preserve definedness:

a. For all $v \in \mathrm{V}, \operatorname{def}(\widehat{\mathrm{VAR}}(v))$.

b. If for all $v \in \mathrm{V}, \operatorname{def}(f v)$, then $\operatorname{def}(\widehat{\mathrm{LAM}}(f))$.

c. If $\operatorname{def}\left(l_{1}\right)$ and $\operatorname{def}\left(l_{2}\right)$, then $\operatorname{def}\left(\widehat{\operatorname{APP}}\left(l_{1}, l_{2}\right)\right)$.

Proof: Straightforward verification in each case.

Lemma 11 Let $m \in \Lambda$ and $\rho \in\left[\mathrm{V} \rightarrow D_{\mathrm{r}}\right]$ be such that for all $x \in F V(m)$, there exists an $l$ with $\rho(x)=\uparrow l$ and $\operatorname{def}(l)$. Then,

a. If $m \in \mathcal{N}_{\text {at }}$, then $\exists l \in \widehat{\Lambda}$. $\llbracket m \rrbracket_{\mathrm{r}} \rho=\uparrow l \wedge \operatorname{def}(l)$.

b. If $m \in \mathcal{N}_{\mathrm{nf}}$, then $\operatorname{def}\left(\downarrow\left(\llbracket m \rrbracket_{\mathrm{r}} \rho\right)\right)$.

Proof: By simultaneous rule induction on $\vdash_{\mathrm{at}} \cdot$ and $\vdash_{\mathrm{nf}} \cdot$. The relevant cases are:

Case $\vdash_{\text {at }} \operatorname{VAR}(x)$ : Then $\llbracket m \rrbracket_{\mathrm{r}} \rho=\rho(x)$, and $x \in F V(m)$, so the result follows directly from the assumption on $\rho$.

Case $\vdash_{\text {at }} \operatorname{APP}\left(m_{1}, m_{2}\right)$ because $\vdash_{\text {at }} m_{1}$ and $\vdash_{\text {nf }} m_{2}$ : By $\operatorname{IH}($ a $)$ on the first premise, there exists an $l_{1}$ such that $\llbracket m_{1} \rrbracket_{\mathrm{r}} \rho=\uparrow l_{1}$ and $\operatorname{def}\left(l_{1}\right)$. Therefore, $\llbracket m \rrbracket_{\mathrm{r}} \rho=$ $\uparrow\left(\widehat{\operatorname{APP}}\left(l_{1}, \downarrow\left(\llbracket m_{2} \rrbracket_{\mathrm{r}} \rho\right)\right)\right)$. Take $l_{2}=\downarrow\left(\llbracket m_{2} \rrbracket_{\mathrm{r}} \rho\right)$ and $l=\widehat{\operatorname{APP}}\left(l_{1}, l_{2}\right)$. By $\operatorname{IH}(\mathrm{b})$ on the second premise, $\operatorname{def}\left(l_{2}\right)$, so by Lemma $10(\mathrm{c}), \operatorname{def}(l)$, as required.

Case $\vdash_{\text {nf }} m$ because $\vdash_{\text {at }} m$ : By $\operatorname{IH}\left(\right.$ a) on the premise, $\llbracket m \rrbracket_{\mathrm{r}} \rho=\uparrow l$, with $\operatorname{def}(l)$. But $\downarrow(\uparrow l)=l$, so also $\operatorname{def}\left(\downarrow \llbracket m \rrbracket_{\mathrm{r}} \rho\right)$.

Case $\vdash_{\text {nf }} \operatorname{LAM}\left(x, m_{0}\right)$ because $\vdash_{\text {nf }} m_{0}$ : Expanding the definition of $\downarrow$ for the functional case, we have to show that $\operatorname{def}\left(\widehat{\mathrm{LAM}}\left(\lambda x . \downarrow\left(\llbracket m_{0} \rrbracket_{\mathrm{r}} \rho[x \mapsto \uparrow(\widehat{\mathrm{VAR}}(x))]\right)\right)\right)$. By Lemma $10(\mathrm{~b})$, it suffices to show that, for every $v \in \mathrm{V}, \operatorname{def}\left(\downarrow\left(\llbracket m_{0} \rrbracket_{\mathrm{r}} \rho^{\prime}\right)\right)$, where $\rho^{\prime}=\rho[x \mapsto \uparrow(\widehat{\operatorname{VAR}}(v))]$. This follows from IH(b) on the premise, if for every $x^{\prime} \in F V\left(m_{0}\right)$, there exists an $l$, such that $\rho^{\prime}\left(x^{\prime}\right)=\uparrow l$ and $\operatorname{def}(l)$. But for $x^{\prime} \neq x$, we must have $x^{\prime} \in F V(m)$, so this follows from the assumption on $\rho$; and for $x^{\prime}=x$, it follows from Lemma 10(a).

Theorem 2 (semantic correctness) norm $_{\Delta}$ from Definition 2 is a normalization function on $\Lambda^{\Delta}$, i.e.,

a. (soundness) If $\operatorname{norm}_{\Delta}(m)=\left\lfloor m^{\prime}\right\rfloor$, then $m^{\prime} \in \Lambda^{\Delta}, m^{\prime} \leftrightarrow m$, and $m^{\prime} \in \mathcal{N}_{\mathrm{nf}}$. 
b. (identification) If $m \leftrightarrow m^{\prime}$, then $\operatorname{norm}_{\Delta}(m)=\operatorname{norm}_{\Delta}\left(m^{\prime}\right)$.

c. (completeness) If for some $m^{\prime} \in \mathcal{N}_{\mathrm{nf}}, m^{\prime} \leftrightarrow m$, then $\operatorname{norm}_{\Delta}(m) \neq \perp$.

Proof: (Soundness) Let $\rho_{0}=\lambda x^{\mathrm{V}} \cdot \uparrow(\widehat{\operatorname{VAR}}(x))$, and let $\theta_{0}$ be the substitution mapping every $x$ in $\Delta$ to $\operatorname{VAR}(x)$. By Lemma $5($ a), for every $x \in \Delta, \widehat{\operatorname{VAR}}(x) \lesssim \Delta$ $\operatorname{VAR}(x)=\theta_{0}(x)$, and hence by Lemma $8\left(\right.$ a),$\rho_{0}(x) \lesssim^{\Delta} \theta_{0}(x)$. By Lemma 9 , we then get that $\llbracket m \rrbracket_{\mathrm{r}} \rho_{0} \lesssim^{\Delta} m\left[\theta_{0}\right] \leftrightarrow m$, and thus, by Lemma $8(\mathrm{~b}), \downarrow\left(\llbracket m \rrbracket_{\mathrm{r}} \rho_{0}\right) \lesssim \Delta_{\text {nf }} m$. Assume now that $\operatorname{norm}_{\Delta}(m)=\downarrow\left(\llbracket m \rrbracket_{\mathrm{r}} \rho_{0}\right) \sharp \Delta=\left\lfloor m^{\prime}\right\rfloor$. Taking $n=\sharp \Delta$ in Definition 3, we can then immediately read off that $m^{\prime}$ has the required properties.

(Identification) This follows directly from model soundness (Lemma 2), since the residualizing model is indeed a model.

(Completeness) Using Lemma 10(a), we see that $\rho_{0}$ satisfies the condition on $\rho$ in Lemma 11. Hence, by part (b) of the latter lemma and Definition $4, \downarrow\left(\llbracket m^{\prime} \rrbracket_{\mathrm{r}} \rho_{0}\right) \sharp \Delta \neq$ $\perp$. And thus, again by model soundness, also $\operatorname{norm}_{\Delta}(m)=\downarrow\left(\llbracket m \rrbracket_{\mathrm{r}} \rho_{0}\right) \sharp \Delta \neq \perp$.

Note that the correctness theorem does not completely pin down the behavior of the normalizer: the soundness specification allows it to return any valid $\alpha$ variant of the normal form, such as normalizing the term $\operatorname{LAM}(\mathrm{x}, \operatorname{LAM}(\mathrm{y}, \operatorname{VAR}(\mathrm{y})))$ to $\operatorname{LAM}\left(\mathrm{g}_{0}, \operatorname{LAM}\left(\mathrm{g}_{0}, \operatorname{VAR}\left(\mathrm{g}_{0}\right)\right)\right)$. Conversely, completeness says only that if a term has a $\beta$-normal form, the normalizer will also find one, though not necessarily the same one.

It would also be possible to adopt a "tight" specification of normal forms, requiring them to also be $\alpha$-normal, such as the current de Bruijn-level naming. Then, a term can have at most one normal form, and the normalizer will in fact find exactly that one when it exists - which would allow us to combine soundness and completeness into a single statement.

\section{An implementation of the construction}

\subsection{Syntax and semantics of an ML-like call-by-value language}

As our implementation language, we take a small fragment of Standard ML [MTHM97]. We deliberately choose an eager language, whose finer control over lifting allows us to mirror all the semantic constructions almost exactly (i.e., up to isomorphism). Any necessary laziness can be easily added by explicit thunking. On the other hand, working in an inherently lazy language, such as Haskell, would make it harder to work with, e.g., the set of $\lambda$-terms as a datatype, without also including spurious infinite and partially-defined elements.

Syntax The fragment is parameterized by a sequence of recursive datatype declarations, each of the form

$$
\text { datatype } d t^{i}=I n_{1}^{i} \text { of } \tau_{11}^{i} * \cdots * \tau_{1 n_{1}}^{i}|\cdots| I n_{k}^{i} \text { of } \tau_{k 1}^{i} * \cdots * \tau_{k n_{k}}^{i} \text {, }
$$

where ML types $\tau$ are given by the grammar,

$$
\tau::=\text { unit } \mid \text { int } \mid \text { bool } \mid \text { string }\left|\tau_{1}->\tau_{2}\right| d t^{i} .
$$




$$
\begin{aligned}
& \overline{\Gamma, x: \tau \vdash x: \tau} \quad \overline{\Gamma \vdash \underline{n}: \text { int }} \quad \overline{\Gamma \vdash " v ": \text { string }} \overline{\Gamma \vdash(): \text { unit }} \\
& \frac{\Gamma \vdash e_{1}: \text { int } \quad \Gamma \vdash e_{2}: \text { int }}{\Gamma \vdash e_{1}+e_{2}: \text { int }} \quad \frac{\Gamma \vdash e_{1}: \tau \quad \Gamma \vdash e_{2}: \tau}{\Gamma \vdash e_{1}=e_{2}: \text { bool }}(\tau \text { ground }) \\
& \Gamma \vdash e \text { : int } \\
& \Gamma \vdash \text { "g"^Int.toString } e \text { : string } \\
& \frac{\Gamma \vdash e: \tau}{\Gamma \vdash \text { fn }()=>: \text { unit-> }} \quad \frac{\Gamma, x: \tau_{1} \vdash e: \tau_{2}}{\Gamma \vdash \mathrm{fn} x=>: \tau_{1}>\tau_{2}} \quad \frac{\Gamma \vdash e_{1}: \tau_{1}->\tau_{2} \quad \Gamma \vdash e_{2}: \tau_{1}}{\Gamma \vdash e_{1} e_{2}: \tau_{2}} \\
& \frac{\Gamma \vdash e_{1}: \tau_{j 1}^{i} \quad \cdots \quad \Gamma \vdash e_{n}: \tau_{j n_{j}}^{i}}{\Gamma \vdash I n_{j}^{i}\left(e_{1}, \ldots, e_{n_{j}}\right): d t^{i}} \\
& \Gamma \vdash e: d t^{i}
\end{aligned}
$$

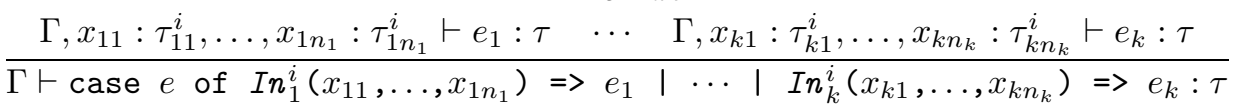

$$
\begin{aligned}
& \frac{\Gamma \vdash e_{1}: \text { bool } \quad \Gamma \vdash e_{2}: \tau \quad \Gamma \vdash e_{3}: \tau}{\Gamma \vdash \text { if } e_{1} \text { then } e_{2} \text { else } e_{3}: \tau} \\
& \frac{\Gamma, f: \tau_{1}->\tau_{2}, x: \tau_{1} \vdash e_{1}: \tau_{2} \quad \Gamma, f: \tau_{1}->\tau_{2} \vdash e_{2}: \tau}{\Gamma \vdash \text { let fun } f\left(x: \tau_{1}\right): \tau_{2}=e \text { in } e_{2} \text { end }: \tau}
\end{aligned}
$$

Figure 1: Typing rules for the ML fragment

The datatypes cannot be mutually recursive, but may be cumulative, i.e., later declarations may refer to earlier ones. We say that a type is ground if it does not contain - directly, or indirectly (through a datatype declaration) - any function spaces. For notational simplicity, we assume that the set of $\lambda$-term variable names, $\mathrm{V}$, is identified with the set of ML character strings.

The syntax of ML expressions is then

$$
\begin{aligned}
e::= & x|\underline{n}| " v "|()| e_{1}+e_{2}\left|e_{1}=e_{2}\right| " g " \wedge \text { Int.toString } e \mid \\
& \text { fn }()=>\mid \text { fn } x=>\left|e_{1} e_{2}\right| \operatorname{In}_{j}^{i}\left(e_{1}, \ldots, e_{n}\right) \mid \\
& \text { case } e \text { of } \operatorname{In}_{1}^{i}\left(x_{11}, \ldots, x_{1 n_{1}}\right)=>e_{1}|\ldots| \operatorname{In} n_{k}^{i}\left(x_{k 1}, \ldots, x_{k n_{k}}\right) \Rightarrow e_{k} \\
& \text { if } e_{1} \text { then } e_{2} \text { else } e_{3} \mid \text { let fun } f\left(x: \tau_{1}\right): \tau_{2}=e_{1} \text { in } e_{2} \text { end }
\end{aligned}
$$

where $x$ and $f$ range over ML variable names.

Typing We only consider well-typed ML expressions, as captured by the judgement $x_{1}: \tau_{1}, \ldots, x_{n}: \tau_{n} \vdash e: \tau$, asserting that $e$ is of type $\tau$, with free variables $x_{1}, \ldots, x_{n}$ of types $\tau_{1}, \ldots, \tau_{n}$. The typing rules are shown in Figure 1

Denotational semantics For the meaning of ML types, we take

$$
\begin{gathered}
\llbracket \text { unit } \rrbracket^{\mathrm{ml}}=\mathbf{1}=\{*\} \quad \llbracket \text { int } \rrbracket^{\mathrm{ml}}=\mathbb{Z} \quad \llbracket \text { bool } \rrbracket^{\mathrm{ml}}=\mathbb{B} \quad \llbracket \text { string } \rrbracket^{\mathrm{ml}}=\mathrm{V} \\
\llbracket \tau_{1}->\tau_{2} \rrbracket^{\mathrm{ml}}=\left[\llbracket \tau_{1} \rrbracket^{\mathrm{ml}} \rightarrow \llbracket \tau_{2} \rrbracket_{\perp}^{\mathrm{ml}}\right] \quad \llbracket d t^{i} \rrbracket^{\mathrm{ml}}=S^{i},
\end{gathered}
$$




$$
\begin{aligned}
& \llbracket x \rrbracket^{\mathrm{ml}} \xi=\lfloor\xi(x)\rfloor \quad \llbracket \underline{n} \rrbracket^{\mathrm{ml}} \xi=\lfloor n\rfloor \quad \llbracket " v^{\prime \prime} \rrbracket^{\mathrm{ml}} \xi=\lfloor v\rfloor \quad \llbracket() \rrbracket^{\mathrm{ml}} \xi=\lfloor *\rfloor \\
& \llbracket e_{1}+e_{2} \rrbracket^{\mathrm{ml}} \xi=\llbracket e_{1} \rrbracket^{\mathrm{ml}} \xi \star \lambda n_{1}^{\mathbb{Z}} \cdot \llbracket e_{2} \rrbracket^{\mathrm{ml}} \xi \star \lambda n_{2}^{\mathbb{Z}} \cdot\left\lfloor n_{1}+n_{2}\right\rfloor \\
& \llbracket e_{1}=e_{2} \rrbracket^{\mathrm{ml}} \xi=\llbracket e_{1} \rrbracket^{\mathrm{ml}} \xi \star \lambda a_{1}^{\llbracket \tau \rrbracket^{\mathrm{ml}}} \cdot \llbracket e_{2} \rrbracket^{\mathrm{ml}} \xi \star \lambda a_{2}^{\llbracket \tau \rrbracket^{\mathrm{ml}}} \cdot\left\lfloor a_{1}=a_{2}\right\rfloor \\
& \llbracket " \mathrm{~g} \text { "^Int.toString } e \rrbracket^{\mathrm{ml}} \xi=\llbracket e \rrbracket^{\mathrm{ml}} \xi \star \lambda n^{\mathbb{Z}} \text {. }\lfloor\mathrm{g} \underline{n}\rfloor \\
& \llbracket \text { fn }()=>e \rrbracket^{\mathrm{ml}} \xi=\left\lfloor\lambda u^{1} \cdot \llbracket e \rrbracket^{\mathrm{ml}} \xi\right\rfloor \quad \llbracket \mathrm{fn} x=>e \rrbracket^{\mathrm{ml}} \xi=\left\lfloor\lambda a^{\llbracket \tau_{1} \rrbracket^{\mathrm{ml}}} \cdot \llbracket e \rrbracket^{\mathrm{ml}} \xi[x \mapsto a]\right\rfloor
\end{aligned}
$$

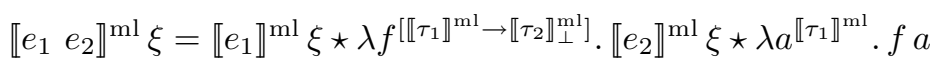

$$
\begin{aligned}
& \llbracket \operatorname{In}_{j}^{i}\left(e_{1}, \ldots, e_{n}\right) \rrbracket^{\mathrm{ml}} \xi= \\
& \llbracket e_{1} \rrbracket^{\mathrm{ml}} \xi \star \lambda a_{1}^{\llbracket \tau_{j 1}^{i} \rrbracket^{\mathrm{ml}}} \cdot \cdots \cdot \llbracket e_{n} \rrbracket^{\mathrm{ml}} \xi \star \lambda a_{n}^{\llbracket \tau_{j n}^{i} \rrbracket^{\mathrm{ml}}} \cdot\left\lfloor\iota_{I n_{j}^{i}}\left(a_{1}, \ldots, a_{n}\right)\right\rfloor \\
& \text { 『case } e \text { of } \operatorname{In} n_{1}^{i}\left(x_{11}, \ldots, x_{1 n_{1}}\right) \Rightarrow e_{1}|\ldots| I n_{k}^{i}\left(x_{k 1}, \ldots, x_{k n_{k}}\right) \Rightarrow e_{k} \rrbracket^{\mathrm{ml}} \xi= \\
& \llbracket e \rrbracket^{\mathrm{ml}} \xi \star \lambda s^{S^{i}} \text {. case } s \text { of }\left\{\begin{array}{c}
{ }^{{ } I n_{1}^{i}}\left(a_{1}, \ldots, a_{n_{1}}\right) \rightarrow \llbracket e_{1} \rrbracket^{\mathrm{ml}} \xi\left[x_{11} \mapsto a_{1}, \ldots, x_{1 n_{1}} \mapsto a_{n_{1}}\right] \\
\vdots \\
{ }{ }_{I n_{k}^{i}}\left(a_{1}, \ldots, a_{n_{k}}\right) \rightarrow \llbracket e_{k} \rrbracket^{\mathrm{ml}} \xi\left[x_{k 1} \mapsto a_{1}, \ldots, x_{k n_{k}} \mapsto a_{n_{k}}\right]
\end{array}\right. \\
& \text { 【if } e_{1} \text { then } e_{2} \text { else } e_{3} \rrbracket^{\mathrm{ml}} \xi=\llbracket e_{1} \rrbracket^{\mathrm{ml}} \xi \star \lambda b^{\mathbb{B}} \text {. case } b \text { of }\left\{\begin{array}{l}
\mathrm{t} \rightarrow \llbracket e_{2} \rrbracket^{\mathrm{ml}} \xi \\
\mathrm{ff} \rightarrow \llbracket e_{3} \rrbracket^{\mathrm{ml}} \xi
\end{array}\right. \\
& \text { 【let fun } f\left(x: \tau_{1}\right): \tau_{2}=e_{1} \text { in } e_{2} \text { end } \rrbracket^{\mathrm{ml}} \xi= \\
& \llbracket e_{2} \rrbracket^{\mathrm{ml}} \xi\left[f \mapsto \operatorname{fix}(\underbrace{\lambda \theta^{\left.\llbracket \llbracket \tau_{1} \rrbracket^{\mathrm{ml}} \rightarrow \llbracket \tau_{2} \rrbracket_{\perp}^{\mathrm{ml}}\right]} \cdot \lambda a^{\llbracket \tau_{1} \rrbracket^{\mathrm{ml}}} \cdot \llbracket e_{1} \rrbracket^{\mathrm{ml}} \xi[f \mapsto \theta, x \mapsto a]}_{\Theta_{f}})\right]
\end{aligned}
$$

Figure 2: Denotational semantics of the ML fragment.

where, for each $d t^{i}$,

$$
i_{d t^{i}}: S^{i} \cong\left(\llbracket \tau_{11}^{i} \rrbracket^{\mathrm{ml}} \times \cdots \times \llbracket \tau_{1 n_{1}}^{i} \rrbracket^{\mathrm{ml}}\right)+\cdots+\left(\llbracket \tau_{k 1}^{i} \rrbracket^{\mathrm{ml}} \times \cdots \times \llbracket \tau_{k n_{k}}^{i} \rrbracket^{\mathrm{ml}}\right)
$$

is a minimal-invariant solution to the evident predomain equation. We write

$$
\iota_{I n_{j}^{i}}\left(a_{1}, \ldots, a_{n}\right)=i_{d t^{i}}^{-1}\left(i n_{j}\left(a_{1}, \ldots, a_{n}\right)\right)
$$

for the constructor functions. Any element of $S^{i}$ can thus be uniquely written as the image of a constructor function.

When all the $\tau_{.}^{i}$ (and hence also $d t^{i}$ ) are ground, the least solution $S^{i}$ will again be a set (discrete cpo), and could be constructed using standard set-theoretic methods. In the general case, one must use, e.g., the domain-theoretic inverse-limit construction, straightforwardly adapted to predomains.

The meaning of ML expressions is defined by induction on the typing derivation; for conciseness we write only the expressions themselves. The semantics is structured such that if $\Gamma \vdash e: \tau$ and for all $\left(x_{i}: \tau_{i}\right) \in \Gamma, \xi\left(x_{i}\right) \in \llbracket \tau_{i} \rrbracket^{\mathrm{ml}}$, then $\llbracket e \rrbracket^{\mathrm{ml}} \xi \in \llbracket \tau \rrbracket_{\perp}^{\mathrm{ml}}$. The full semantics is shown in Figure 2

For notational convenience in the following, we will assume that all function names 


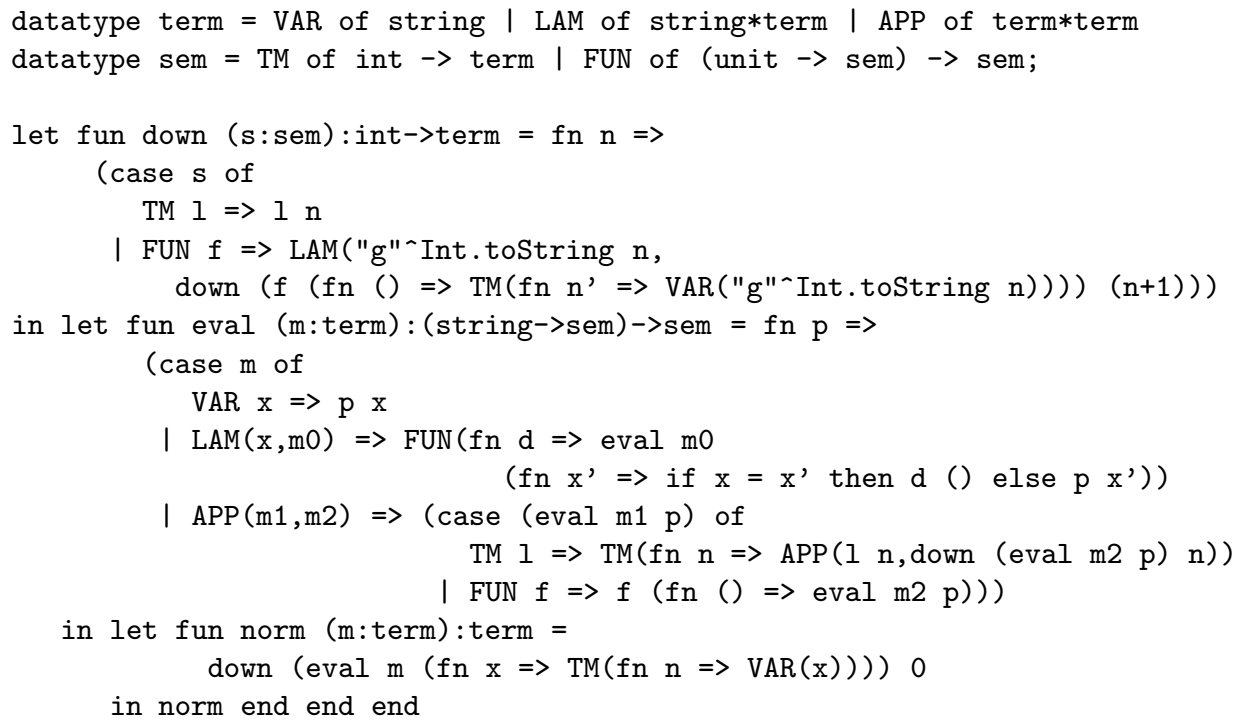

Figure 3: The normalization algorithm, NORM.

$f$ in a program are distinct. For a fixed program, we can then unambiguously use $\theta_{f}=\operatorname{fix}\left(\Theta_{f}\right)$ to refer to the denotation of $f$ in the let fun-construct.

Evaluation semantics We say that a complete program is a closed expression of type $\tau_{1} \rightarrow \tau_{2} \rightarrow>->\tau_{n} \rightarrow>\tau_{0}(n \geq 0)$, where each $\tau_{i}$ is a ground type. For such types, let $C_{\tau}=\llbracket \tau \rrbracket^{\mathrm{ml}}$ denote the set of values underlying $\tau$, e.g., $C_{\text {int }}=\mathbb{Z}$. A complete program $e: \tau_{1} \rightarrow \tau_{2} \rightarrow \cdots \rightarrow \tau_{n} \rightarrow \tau_{0}$ then determines a computable partial function $e \bullet(\cdot): C_{\tau_{1}} \times \cdots \times C_{\tau_{n}} \rightarrow C_{\tau_{0}}$, given, e.g., by

$$
e \bullet\left(c_{1}, \ldots, c_{n}\right)=c_{0} \text { iff }\left(e \underline{c_{1}} \cdots \underline{c_{n}}\right) \Downarrow^{\mathrm{ml}} \underline{c_{0}},
$$

where $\Downarrow^{\mathrm{ml}}$ is the usual big-step operational semantics of expressions, and $\underline{c}$ denotes the syntactic representation of the value $c$.

Theorem 3 (computational adequacy of denotational semantics) For a complete $M L$ program $e, e \bullet\left(c_{1}, \ldots, c_{n}\right)=c_{0}$ iff $\llbracket e \rrbracket^{\mathrm{ml} \emptyset} \emptyset \lambda f_{1} . f_{1} c_{1} \star \cdots \star \lambda f_{n} . f_{n} c_{n}=\left\lfloor c_{0}\right\rfloor$.

Proof: Modulo trivial syntactic differences, an equivalent formulation of the semantics in terms of strict functions between pointed cpos, rather than general ones between cpos, and the obvious generalization to multiple (non-mutually recursive) datatypes, this is shown in, e.g., [Pit96, Section 5]. The primary difficulty is, of course, the definition of the logical relation at types $d t^{i}$, which is again achieved by exploiting the minimal-invariant properties of the $\left(S^{i}, i_{d} d t^{i}\right)$.

\subsection{The normalization algorithm}

The concrete representation of the normalization algorithm, with many of the auxiliary definitions inlined, is shown in Figure 3. We have taken $d t^{1}=$ term with three 
constructors $I n_{1}^{1}=$ VAR, $I n_{2}^{1}=\mathrm{LAM}$, and $I n_{3}^{1}=$ APP. Note that term is a ground type. To keep the notation concise, we assume that the chosen predomain-equation solution coincides exactly with our representation of terms, i.e., $\llbracket$ term $\rrbracket^{\mathrm{ml}}=\Lambda, \iota_{\text {LAM }}(v, m)=$ $\operatorname{LAM}(v, m)$, etc. Similarly, we have instantiated $d t^{2}$ as the type sem, with constructors TM and FUN. We write $S=\llbracket \mathrm{sem} \rrbracket^{\mathrm{ml}}$, but here we do not a priori require that $S_{\perp}=D_{\mathrm{r}}$.

Since ML is a call-by-value language, we must simulate the implicit call-by-name nature of the residualizing semantics using thunking. We have defined sem so that $\llbracket \mathrm{sem} \rrbracket_{\perp}^{\mathrm{ml}} \cong D_{\mathrm{r}}$; then semantic functions with codomain $D_{\mathrm{r}}$ can be represented directly as ML functions into sem, while functions with domain $D_{\mathrm{r}}$ are represented as ML functions with source type unit $\rightarrow$ sem. As an optimization, however, the strict function $\downarrow: D_{\mathrm{r}} \rightarrow \widehat{\Lambda}$ is represented as simply an ML function on sem.

It is easy to check that the top-level expression, NORM : term $\rightarrow$ term, is a welltyped complete program in our sense.

Examples The following examples illustrate how the program behaves. Let $S A \equiv$ $\operatorname{LAM}(\mathrm{x}, \operatorname{APP}(\operatorname{VAR}(\mathrm{x}), \operatorname{VAR}(\mathrm{x})))$ and Omega $\equiv \operatorname{APP}(S A, S A)$.
a. $N O R M \bullet(O m e g a)$ diverges.
b. $N O R M \bullet(\operatorname{APP}(\operatorname{LAM}(\mathrm{x}, \operatorname{LAM}(\mathrm{x}, \operatorname{VAR}(\mathrm{x})))$, Omega $))=\operatorname{LAM}\left(\mathrm{g}_{0}, \operatorname{VAR}\left(\mathrm{g}_{0}\right)\right)$.
c. $N O R M \bullet\left(\operatorname{LAM}\left(\mathrm{y}, \operatorname{LAM}\left(\mathrm{g}_{4}, \operatorname{VAR}(\mathrm{z})\right)\right)\right)=\operatorname{LAM}\left(\mathrm{g}_{0}, \operatorname{LAM}\left(\mathrm{g}_{1}, \operatorname{VAR}(\mathrm{z})\right)\right)$.

Let us now formally relate the abstract and concrete constructions. To obtain a perfect correspondence between semantic term generators and their implementation, we choose $\mathrm{N}=\mathbb{Z}$, with $\operatorname{gen}(n)=\mathrm{g} \underline{n}=\mathrm{g}_{n}$ when $n \geq 0$, e.g., gen(13)= g13; then $\llbracket$ int $\rightarrow$ term $\rrbracket^{\mathrm{ml}}=\widehat{\Lambda}$. Recall that we had $D_{\mathrm{r}} \cong\left(\widehat{\Lambda}+\left[D_{\mathrm{r}} \rightarrow D_{\mathrm{r}}\right]\right)_{\perp}$, while $\llbracket \mathrm{sem} \rrbracket^{\mathrm{ml}}=$ $S \cong \widehat{\Lambda}+\left[\left[\mathbf{1} \rightarrow S_{\perp}\right] \rightarrow S_{\perp}\right]$. We can then show:

Lemma 12 There exists an isomorphism $i_{D S}: D_{\mathrm{r}} \cong S_{\perp}$, satisfying:
a. For all $l \in \widehat{\Lambda}, i_{D S}(\operatorname{tm}(l))=\left\lfloor\iota_{\mathrm{TM}}(l)\right\rfloor$.
b. For all $f \in\left[D_{\mathrm{r}} \rightarrow D_{\mathrm{r}}\right], i_{D S}(\operatorname{fun}(f))=\left\lfloor\iota_{\mathrm{FUN}}\left(\lambda t^{\left[\mathbf{1} \rightarrow S_{\perp}\right]} \cdot i_{D S}\left(f\left(i_{D S}^{-1}(t *)\right)\right)\right)\right\rfloor$.
c. $i_{D S}\left(\perp_{D_{\mathrm{r}}}\right)=\perp_{S_{\perp}}$.

Proof: See Appendix B.1.

We can also state three lemmas, relating the central domain-theoretic functions to the denotations of their syntactic counterparts:

Lemma 13 For all $d \in D_{\mathrm{r}}$ and $n \in \mathbb{Z}, \downarrow d n=i_{D S}(d) \star \lambda s^{S} . \theta_{\text {down }} s \star \lambda l^{\widehat{\Lambda}}$. $l n$.

Proof: By fixed-point induction on $\Phi \times \Theta_{\text {down }}$ (where $\Phi$ is as in the proof of Lemma 8), using the predicate $R \subseteq\left[D_{\mathrm{r}} \rightarrow \widehat{\Lambda}\right] \times\left[S \rightarrow \widehat{\Lambda}_{\perp}\right]$ defined by

$$
R=\left\{(\varphi, \theta) \mid \forall d \in D_{\mathrm{r}}, n \in \mathbb{Z} . \varphi d n=i_{D S}(d) \star \lambda s^{S} . \theta s \star \lambda l^{\widehat{\Lambda}} . l n\right\} .
$$

We aim to establish that $\left(\operatorname{fix}(\Phi), \operatorname{fix}\left(\Theta_{\text {down }}\right)\right) \in R$. It is straightforward to verify that $R$ is pointed and inclusive. Assume that $(\varphi, \theta) \in R$; we then must show that $\left(\Phi(\varphi), \Theta_{\text {down }}(\theta)\right) \in R$. Accordingly, let arbitrary $d$ and $n$ be given, and consider $d$ : 
Case $d=\perp_{D_{\mathrm{r}}}$ : By Lemma 12(c), $i_{D S}(d)=\perp_{S_{\perp}}$, and so

$$
\begin{aligned}
& i_{D S}(d) \star \lambda s^{S} . \Theta_{\text {down }}(\theta) s \star \lambda l^{\widehat{\Lambda}} . l n \\
& =\perp_{\Lambda_{\perp}} .
\end{aligned}
$$

Similarly, $\Phi(\varphi) d n=\perp_{\widehat{\Lambda}} n=\perp_{\Lambda_{\perp}}$.

Case $d=\operatorname{tm}(l)$ : Let $\xi=\emptyset\left[\right.$ down $\left.\mapsto \theta, \mathrm{s} \mapsto \iota_{\mathrm{TM}}(l)\right]$; we calculate:

$$
\begin{aligned}
& i_{D S}(d) \star \lambda s^{S} . \Theta_{\text {down }}(\theta) s \star \lambda l^{\widehat{\Lambda}} . l n \\
& =i_{D S}(\operatorname{tm}(l)) \star \lambda s^{S} . \Theta_{\text {down }}(\theta) s \star \lambda l^{\widehat{\Lambda}} . l n \\
& =\left\lfloor\iota_{\mathrm{TM}}(l)\right\rfloor \star \lambda s^{S} . \Theta_{\text {down }}(\theta) s \star \lambda l^{\widehat{\Lambda}} . l n \\
& =\Theta_{\text {down }}(\theta)\left(\iota_{\mathrm{TM}}(l)\right) \star \lambda l^{\widehat{\Lambda}} . l n \\
& =\llbracket \text { fn } \mathrm{n} \Rightarrow(\text { case } \mathrm{s} \text { of } \mathrm{TM} l \Rightarrow l \mathrm{n} \mid \ldots) \rrbracket^{\mathrm{ml}} \xi \star \lambda l^{\widehat{\Lambda}} . l n \\
& =\llbracket 1 \mathrm{n} \rrbracket^{\mathrm{ml}} \xi[\mathrm{n} \mapsto n, 1 \mapsto l] \\
& =l n
\end{aligned}
$$

Similarly, $\Phi(\varphi) d n=\Phi(\varphi)(\operatorname{tm}(l)) n=l n$.

Case $d=$ fun $(f)$ : Let $\xi=\emptyset\left[\right.$ down $\left.\mapsto \theta, \mathbf{s} \mapsto \iota_{\mathrm{FuN}}\left(\lambda t . i_{D S}\left(f\left(i_{D S}^{-1}(t *)\right)\right)\right)\right]$ and let $\xi^{\prime}=$ $\xi\left[\mathrm{n} \mapsto n, \mathrm{f} \mapsto\left(\lambda t . i_{D S}\left(f\left(i_{D S}^{-1}(t *)\right)\right)\right)\right] ;$ again,

$$
\begin{aligned}
& i_{D S}(d) \star \lambda s^{S} . \Theta_{\text {down }}(\theta) s \star \lambda l^{\widehat{\Lambda}} . l n \\
& =i_{D S}(\text { fun }(f)) \star \lambda s^{S} . \Theta_{\text {down }}(\theta) s \star \lambda l^{\widehat{\Lambda}} . l n \\
& =\left\lfloor\iota_{\mathrm{FUN}}\left(\lambda t . i_{D S}\left(f\left(i_{D S}^{-1}(t *)\right)\right)\right)\right\rfloor \star \lambda s^{S} . \Theta_{\text {down }}(\theta) s \star \lambda l^{\widehat{\Lambda}} . l n \quad \text { (by Lemma 12(b)) } \\
& =\llbracket \text { fn } \mathrm{n} \Rightarrow(\text { case } \mathrm{s} \text { of } \ldots \mid \text { FUN } \mathrm{f} \Rightarrow \operatorname{LAM} \ldots) \rrbracket^{\mathrm{ml}} \xi \star \lambda l^{\widehat{\Lambda}} . l n \\
& =\llbracket \operatorname{LAM}\left(" g \text { " Int.toString }(\mathrm{n}) \text {, down }(\mathrm{f}(\mathrm{fn}()=>\ldots)(\mathrm{n}+1)) \rrbracket^{\mathrm{ml}} \xi^{\prime}\right. \\
& =\llbracket \text { down }(f(\text { fn }() \Rightarrow \ldots))(\mathrm{n}+1) \rrbracket^{\mathrm{ml}} \xi^{\prime} \star \lambda m^{\Lambda} .\left\lfloor\operatorname{LAM}\left(\mathrm{g}_{n}, m\right)\right\rfloor \\
& =\underbrace{\llbracket f(\mathrm{fn}()=>\ldots) \rrbracket^{\mathrm{ml}} \xi^{\prime}}_{s^{\prime}} \star \lambda s^{S} \cdot \theta s \star \lambda l^{\widehat{\Lambda}} \cdot l(n+1) \star \lambda m^{\Lambda} \cdot\left\lfloor\operatorname{LAM}\left(\mathrm{g}_{n}, m\right)\right\rfloor
\end{aligned}
$$

Now,

$$
\begin{aligned}
& s^{\prime} \\
& =\llbracket \mathrm{f}\left(\mathrm{fn}() \Rightarrow \operatorname{TM}\left(\mathrm{fn} \mathrm{n}^{\prime} \Rightarrow \operatorname{VAR}(" \mathrm{~g} " \wedge \operatorname{Int} \cdot \operatorname{toString}(\mathrm{n}))\right)\right) \rrbracket^{\mathrm{ml}} \xi^{\prime} \\
& =\left\lfloor\left(\lambda t . i_{D S}\left(f\left(i_{D S}^{-1}(t *)\right)\right)\right)\right\rfloor \star \lambda g \cdot\left\lfloor\lambda u \cdot \llbracket \mathrm{TM}(\mathrm{fn} \ldots .) \rrbracket^{\mathrm{ml}} \xi^{\prime}\right\rfloor \star \lambda a . g a \\
& =i_{D S}\left(f\left(i_{D S}^{-1}\left(\left\lfloor\iota_{\mathrm{TM}}\left(\lambda n^{\mathbb{Z}} \cdot\left\lfloor\operatorname{VAR}\left(\mathrm{g}_{n}\right)\right\rfloor\right)\right\rfloor\right)\right)\right) \\
& =i_{D S}\left(f\left(\operatorname{tm}\left(\lambda n^{\prime \mathbb{Z}} \cdot\left\lfloor\operatorname{VAR}\left(\mathrm{g}_{n}\right)\right\rfloor\right)\right)\right) \\
& =i_{D S}\left(f\left(\uparrow\left(\widehat{\operatorname{VAR}}\left(\mathrm{g}_{n}\right)\right)\right)\right)
\end{aligned}
$$

Now, the IH that $(\varphi, \theta) \in R$ says that $\forall d^{\prime}, n^{\prime} . \varphi d^{\prime} n^{\prime}=i_{D S}\left(d^{\prime}\right) \star \lambda s^{S} . \theta s \star \lambda l^{\widehat{\Lambda}} . l n^{\prime}$. Taking $d^{\prime}=f\left(\uparrow\left(\widehat{\operatorname{VAR}}\left(\mathrm{g}_{n}\right)\right)\right)$ and $n^{\prime}=n+1$, we continue the original calculation:

$$
\begin{aligned}
& \llbracket \mathrm{f} \quad(\mathrm{fn}()=>\ldots) \rrbracket^{\mathrm{ml}} \xi^{\prime} \star \lambda s^{S} \cdot \theta s \star \lambda l^{\widehat{\Lambda}} \cdot l(n+1) \star \lambda m^{\Lambda} \cdot\left\lfloor\operatorname{LAM}\left(\mathrm{g}_{n}, m\right)\right\rfloor \\
& =i_{D S}\left(f\left(\uparrow\left(\widehat{\operatorname{VAR}}\left(\mathrm{g}_{n}\right)\right)\right)\right) \star \lambda s^{S} \cdot \theta s \star \lambda l^{\widehat{\Lambda}} \cdot l(n+1) \star \lambda m^{\Lambda} \cdot\left\lfloor\operatorname{LAM}\left(\mathrm{g}_{n}, m\right)\right\rfloor \\
& =\varphi\left(f\left(\uparrow\left(\widehat{\operatorname{VAR}}\left(\mathrm{g}_{n}\right)\right)\right)\right)(n+1) \star \lambda m^{\Lambda} \cdot\left\lfloor\operatorname{LAM}\left(\mathrm{g}_{n}, m\right)\right\rfloor
\end{aligned}
$$

Similarly, 


$$
\begin{aligned}
& \Phi(\varphi) d n \\
& =\Phi(\varphi)(\text { fun }(f)) n \\
& =\widehat{\mathrm{LAM}}\left(\lambda x^{\mathrm{V}} \cdot \varphi(f(\uparrow(\widehat{\mathrm{VAR}}(x))))\right) n \\
& \left.=\varphi\left(f\left(\uparrow\left(\widehat{\operatorname{VAR}}\left(\mathrm{g}_{n}\right)\right)\right)\right)(n+1) \star \lambda m^{\Lambda} \cdot\left\lfloor\operatorname{LAM}\left(\mathrm{g}_{n}, m\right)\right\rfloor \quad \text { (by Def. of } \widehat{\mathrm{LAM}}\right)
\end{aligned}
$$

Lemma 14 For all $m \in \Lambda, \rho \in\left[\mathrm{V} \rightarrow D_{\mathrm{r}}\right]$, and $\varrho \in\left[\mathrm{V} \rightarrow S_{\perp}\right]$, satisfying that $\forall x \in$ $F V(m) \cdot i_{D S}(\rho(x))=\varrho(x), i_{D S}\left(\llbracket m \rrbracket_{\mathrm{r}} \rho\right)=\theta_{\text {eval }} m \star \lambda g . g \varrho$.

Proof: By structural induction on $m$. Let $m, \rho$ and $\varrho$ be given such that $\forall x \in$ $F V(m) \cdot i_{D S}(\rho(x))=\varrho(x)$. Let $\xi=\emptyset\left[\right.$ down $\left.\mapsto \theta_{\text {down }}\right]$. By the fixed-point equation, since $\theta_{\text {eval }}=\operatorname{fix}\left(\Theta_{\text {eval }}\right)$

$\theta_{\text {eval }} m \star \lambda g . g \varrho$

$=\Theta_{\text {eval }}\left(\theta_{\text {eval }}\right) m \star \lambda g . g \varrho$

$=\llbracket \mathrm{fn} \mathrm{p} \Rightarrow($ case $\mathrm{m}$ of $\ldots) \rrbracket^{\mathrm{ml}} \xi\left[\right.$ eval $\left.\mapsto \theta_{\text {eval }}, \mathrm{m} \mapsto m\right] \star \lambda g . g \varrho$

$=\llbracket$ case $\mathrm{m}$ of $\ldots \rrbracket^{\mathrm{ml}} \xi\left[\right.$ eval $\left.\mapsto \theta_{\text {eval }}, \mathrm{m} \mapsto m, \mathrm{p} \mapsto \varrho\right]$

Let $\xi^{\prime}=\xi\left[\right.$ eval $\left.\mapsto \theta_{\text {eval }}, \mathrm{m} \mapsto m, \mathrm{p} \mapsto \varrho\right]$. Consider $m$ :

Case $m=\operatorname{VAR}(x)$ : Then,

$$
\begin{aligned}
& \theta_{\text {eval }} m \star \lambda g . g \varrho \\
& =\llbracket \text { case } \mathrm{m} \text { of } \operatorname{VAR} \mathrm{x} \Rightarrow \mathrm{p} x \quad \mid \ldots \rrbracket^{\mathrm{ml}} \xi^{\prime} \\
& =\llbracket \mathrm{p} \quad \mathrm{x} \rrbracket^{\mathrm{ml}} \xi^{\prime}[\mathrm{x} \mapsto x] \\
& =\varrho(x)
\end{aligned}
$$

Since clearly $x \in F V(m)$, we have $i_{D S}(\rho(x))=\varrho(x)$ by assumption on $\rho$ and $\varrho$. Thus similarly,

$$
\begin{aligned}
& i_{D S}\left(\llbracket m \rrbracket_{\mathrm{r}} \rho\right) \\
& =i_{D S}\left(\llbracket \operatorname{VAR}(x) \rrbracket_{\mathrm{r}} \rho\right) \\
& =i_{D S}(\rho(x)) \\
& =\varrho(x)
\end{aligned}
$$

Case $m=\operatorname{LAM}\left(x, m_{0}\right)$ : Let $\xi^{\prime \prime}=\xi^{\prime}\left[\mathrm{x} \mapsto x, \mathrm{~m} 0 \mapsto m_{0}\right]$. Then,

$$
\begin{aligned}
& \theta_{\text {eval }} m \star \lambda g . g \varrho \\
& =\llbracket \text { case } \mathrm{m} \text { of } \ldots|\operatorname{LAM}(\mathrm{x}, \mathrm{m0}) \Rightarrow \operatorname{FUN}(\ldots)| \ldots \rrbracket^{\mathrm{ml}} \xi^{\prime} \\
& =\llbracket \text { FUN }(\text { fn } \mathrm{d} \Rightarrow \text { eval } \mathrm{mo}(\ldots)) \rrbracket^{\mathrm{ml}} \xi^{\prime \prime} \\
& \left.=\left\lfloor\iota_{\mathrm{FUN}}\left(\lambda t^{1 \rightarrow S_{\perp}} \cdot \llbracket \text { eval m0 (fn } \mathrm{x}^{\prime} \Rightarrow \text { if } \ldots\right) \rrbracket^{\mathrm{ml}} \xi^{\prime \prime}[\mathrm{d} \mapsto t]\right)\right\rfloor \\
& =\left\lfloor\iota_{\mathrm{FUN}}(\underbrace{\lambda t . \theta_{\text {eval }} m_{0} \star \lambda g \cdot g\left(\lambda x^{\prime \mathrm{V}} \cdot \llbracket \text { if } \ldots \rrbracket^{\mathrm{ml}} \xi^{\prime \prime}\left[\mathrm{d} \mapsto t, \mathrm{x}{ }^{\prime} \mapsto x^{\prime}\right]\right)}_{f})\right\rfloor
\end{aligned}
$$

Similarly,

$$
\begin{aligned}
& i_{D S}\left(\llbracket m \rrbracket_{\mathrm{r}} \rho\right) \\
& =i_{D S}\left(\llbracket \operatorname{LAM}\left(x, m_{0}\right) \rrbracket_{\mathrm{r}} \rho\right) \\
& =i_{D S}\left(\phi_{\mathrm{r}}\left(\lambda d^{D_{\mathrm{r}}} \cdot \llbracket m_{0} \rrbracket_{\mathrm{r}} \rho[x \mapsto d]\right)\right) \\
& =i_{D S}\left(\operatorname{fun}\left(\lambda d^{D_{\mathrm{r}}} \cdot \llbracket m_{0} \rrbracket_{\mathrm{r}} \rho[x \mapsto d]\right)\right) \\
& =\left\lfloor\iota_{\mathrm{FUN}}\left(\lambda t \cdot i_{D S}\left(\left(\lambda d^{D_{\mathrm{r}}} \cdot \llbracket m_{0} \rrbracket_{\mathrm{r}} \rho[x \mapsto d]\right)\left(i_{D S}^{-1}(t *)\right)\right)\right)\right\rfloor \\
& =\left\lfloor\iota_{\mathrm{FUN}}(\underbrace{\lambda t \cdot i_{D S}\left(\llbracket m_{0} \rrbracket_{\mathrm{r}} \rho\left[x \mapsto i_{D S}^{-1}(t *)\right]\right)}_{f^{\prime}})\right\rfloor
\end{aligned}
$$


It remains now to prove that $f=f^{\prime}$. Let any $t^{\prime}: \mathbf{1} \rightarrow S_{\perp}$ be given.

Let $\rho_{0}=\rho\left[x \mapsto i_{D S}^{-1}\left(t^{\prime} *\right)\right]$ and $\varrho_{0}=\left(\lambda x^{\prime \mathrm{V}}\right.$. if $\left.\ldots \rrbracket^{\mathrm{ml}} \xi^{\prime \prime}\left[\mathrm{d} \mapsto t^{\prime}, \mathrm{x}^{\prime} \mapsto x^{\prime}\right]\right)$. First we verify that $\rho_{0}$ and $\varrho_{0}$ satisfy the requirements of the IH for $m_{0}$, namely that for all $x^{\prime} \in F V\left(m_{0}\right) \subseteq\{x\} \cup F V(m), i_{D S}\left(\rho_{0}\left(x^{\prime}\right)\right)=\varrho_{0}\left(x^{\prime}\right)$. This is straightforward; first for $x^{\prime}=x$ :

$$
\begin{aligned}
& \varrho_{0}(x) \\
& =\llbracket \text { if } \mathrm{x}=\mathrm{x}, \text { then } \mathrm{d}() \text { else } \mathrm{p} \mathrm{x}^{\prime} \rrbracket^{\mathrm{ml}} \xi^{\prime \prime}\left[\mathrm{d} \mapsto t^{\prime}, \mathrm{x}^{\prime} \mapsto x\right] \\
& =t^{\prime} * \\
& =i_{D S}\left(i_{D S}^{-1}\left(t^{\prime} *\right)\right) \\
& =i_{D S}\left(\rho_{0}(x)\right)
\end{aligned}
$$

Then for any $x^{\prime \prime} \in F V\left(m_{0}\right) \backslash\{x\}$ :

$$
\begin{array}{ll}
\varrho_{0}\left(x^{\prime \prime}\right) & \\
=\llbracket \text { if } \mathrm{x}=\mathrm{x}, \text { then } \mathrm{d}\left(\text { ) else } \mathrm{p} \mathrm{x}^{\prime} \rrbracket^{\mathrm{ml}} \xi^{\prime \prime}\left[\mathrm{d} \mapsto t^{\prime}, \mathrm{x}^{\prime} \mapsto x^{\prime \prime}\right]\right. \\
=\varrho\left(x^{\prime \prime}\right) & \quad \text { (by assumption on } \rho \text { and } \varrho \text { ) } \\
=i_{D S}\left(\rho\left(x^{\prime \prime}\right)\right) & \\
=i_{D S}\left(\rho_{0}\left(x^{\prime \prime}\right)\right) &
\end{array}
$$

Hence,

$$
\begin{aligned}
& f t^{\prime} \\
& =\theta_{\text {eval }} m_{0} \star \lambda g \cdot g \varrho_{0} \\
& =i_{D S}\left(\llbracket m_{0} \rrbracket_{\mathrm{r}} \rho_{0}\right) \\
& =f^{\prime} t^{\prime}
\end{aligned}
$$

Since $t^{\prime}$ was arbitrary, $f=f^{\prime}$.

Case $m=\operatorname{APP}\left(m_{1}, m_{2}\right)$ : Let $\xi^{\prime \prime}=\xi^{\prime}\left[\mathrm{m} 1 \mapsto m_{1}, \mathrm{~m} 2 \mapsto m_{2}\right]$. Then,

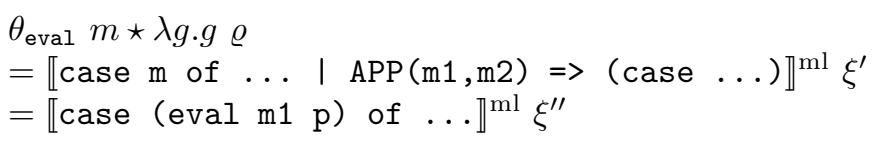

Now,

$$
\begin{aligned}
& \text { đeval m1 p } \rrbracket^{\mathrm{ml}} \xi^{\prime \prime} \\
& =\theta_{\text {eval }} m_{1} \star \lambda g \cdot g \varrho \\
& =i_{D S}\left(\llbracket m_{1} \rrbracket_{\mathrm{r}} \rho\right)
\end{aligned}
$$

Consider $\llbracket m_{1} \rrbracket_{\mathrm{r}} \rho$ :

Case $\llbracket m_{1} \rrbracket_{\mathrm{r}} \rho=\perp_{D_{\mathrm{r}}}$ : Then by Lemma $12(\mathrm{c})$ also $i_{D S}\left(\llbracket m_{1} \rrbracket_{\mathrm{r}} \rho\right)=\perp$, and so $\theta_{\text {eval }} m \star$ $\lambda g . g \varrho=\perp_{S_{\perp}}$.

Similarly,

$$
\begin{aligned}
& i_{D S}\left(\llbracket m \rrbracket_{\mathrm{r}} \rho\right) \\
& =i_{D S}\left(\llbracket \operatorname{APP}\left(m_{1}, m_{2}\right) \rrbracket_{\mathrm{r}} \rho\right) \\
& =i_{D S}\left(\psi_{\mathrm{r}}\left(\perp_{D_{\mathrm{r}}}\right)\left(\llbracket m_{2} \rrbracket_{\mathrm{r}} \rho\right)\right) \\
& =i_{D S}\left(\perp_{\left[D_{\mathrm{r}} \rightarrow D_{\mathrm{r}}\right]}\left(\llbracket m_{2} \rrbracket_{\mathrm{r}} \rho\right)\right) \\
& =i_{D S}\left(\perp_{D_{\mathrm{r}}}\right) \\
& =\perp_{\perp}
\end{aligned}
$$

Case $\llbracket m_{1} \rrbracket_{\mathrm{r}} \rho=\operatorname{tm}\left(l_{1}\right):$ Then, 


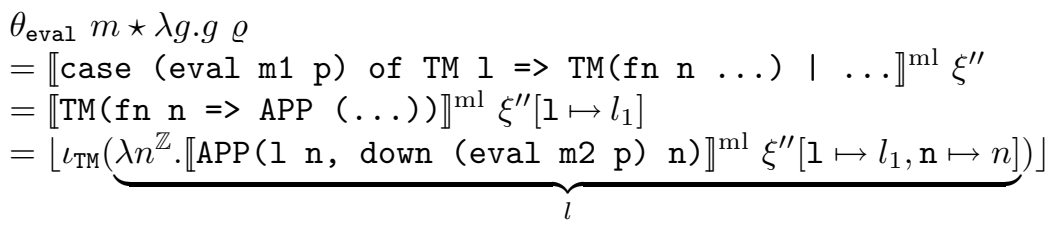

Similarly,

$$
\begin{aligned}
& i_{D S}\left(\llbracket m \rrbracket_{\mathrm{r}} \rho\right) \\
& =i_{D S}\left(\llbracket \operatorname{APP}\left(m_{1}, m_{2}\right) \rrbracket_{\mathrm{r}} \rho\right) \\
& =i_{D S}\left(\psi_{\mathrm{r}}\left(\operatorname{tm}\left(l_{1}\right)\right)\left(\llbracket m_{2} \rrbracket_{\mathrm{r}} \rho\right)\right) \\
& =i_{D S}\left(\uparrow \widehat{\operatorname{APP}}\left(l_{1}, \downarrow\left(\llbracket m_{2} \rrbracket_{\mathrm{r}} \rho\right)\right)\right) \\
& =i_{D S}\left(\operatorname{tm}\left(\widehat{\operatorname{APP}}\left(l_{1}, \downarrow\left(\llbracket m_{2} \rrbracket_{\mathrm{r}} \rho\right)\right)\right)\right) \\
& =\left\lfloor\iota_{\mathrm{TM}}\left(\widehat{\operatorname{APP}}\left(l_{1}, \downarrow\left(\llbracket m_{2} \rrbracket_{\mathrm{r}} \rho\right)\right)\right)\right\rfloor \\
& \left.=\left\lfloor\iota_{\mathrm{TM}}(\underbrace{\lambda n^{\mathbb{Z}} \cdot l_{1} n \star \lambda m_{1}^{\prime} \cdot \downarrow\left(\llbracket m_{2} \rrbracket_{\mathrm{r}} \rho\right) n \star \lambda m_{2}^{\prime} \cdot\left\lfloor\operatorname{APP}\left(m_{1}^{\prime}, m_{2}^{\prime}\right)\right\rfloor}_{l^{\prime}})\right\rfloor \quad \text { (by Def. Lemma } \uparrow\right)
\end{aligned}
$$

Again, it remains to show that $l=l^{\prime}$. Let any $n \in \mathbb{Z}$ be given.

Let $\xi^{\prime \prime \prime}=\xi^{\prime \prime}\left[1 \mapsto l_{1}, \mathrm{n} \mapsto n\right]$; we calculate:

$$
\begin{aligned}
& l n \\
& =\llbracket \operatorname{APP}\left(1 \mathrm{n}, \text { down }(\text { eval m2 p) } \mathrm{n}) \rrbracket^{\mathrm{ml}} \xi^{\prime \prime \prime}\right. \\
& =l_{1} n \star \lambda m_{1}^{\prime} \cdot \llbracket \text { down }\left(\text { eval m2 p) } \rrbracket^{\mathrm{ml}} \xi^{\prime \prime \prime} \star \lambda l_{2} \cdot l_{2} n \star \lambda m_{2}^{\prime} \cdot\left\lfloor\operatorname{APP}\left(m_{1}^{\prime}, m_{2}^{\prime}\right)\right\rfloor\right. \\
& =l_{1} n \star \lambda m_{1}^{\prime} \cdot \theta_{\text {eval }} m_{2} \star \lambda g \cdot g \varrho \star \lambda s \cdot \theta_{\text {down }} s \star \lambda l_{2} \cdot l_{2} n \star \lambda m_{2}^{\prime} \cdot\left\lfloor\operatorname{APP}\left(m_{1}^{\prime}, m_{2}^{\prime}\right)\right\rfloor \\
& =l_{1} n \star \lambda m_{1}^{\prime} \cdot i_{D S}\left(\llbracket m_{2} \rrbracket_{\mathrm{r}} \rho\right) \star \lambda s \cdot \theta_{\text {down }} s \star \lambda l_{2} \cdot l_{2} n \star \lambda m_{2}^{\prime} \cdot\left\lfloor\operatorname{APP}\left(m_{1}^{\prime}, m_{2}^{\prime}\right)\right\rfloor \\
& =l_{1} n \star \lambda m_{1}^{\prime} \cdot \downarrow\left(\llbracket m_{2} \rrbracket_{\mathrm{r}} \rho\right) n \star \lambda m_{2}^{\prime} \cdot\left\lfloor\operatorname{APP}\left(m_{1}^{\prime}, m_{2}^{\prime}\right)\right\rfloor \quad\left(\text { by IH on } m_{2}\right) \\
& =l^{\prime} n
\end{aligned}
$$

Since $n$ was arbitrary, $l=l^{\prime}$.

Case $\llbracket m_{1} \rrbracket_{\mathrm{r}} \rho=$ fun $(f)$ : Then by Lemma $12(\mathrm{~b})$, we have $i_{D S}\left(\llbracket m_{1} \rrbracket_{\mathrm{r}} \rho\right)=$ $\left\lfloor\iota_{\mathrm{FUN}}\left(\lambda t^{\mathbf{1 \rightarrow} S_{\perp}} . i_{D S}\left(f\left(i_{D S}^{-1}(t *)\right)\right)\right)\right\rfloor$. Thus,

$$
\begin{aligned}
& \theta_{\text {eval }} m \star \lambda g . g \varrho \\
& =\llbracket \text { case }(\text { eval } \mathrm{m} 1 \mathrm{p}) \text { of } \ldots \mid \text { | FUN } \mathrm{f}=\mathrm{f}(\mathrm{fn} \ldots) \rrbracket^{\mathrm{ml}} \xi^{\prime \prime}
\end{aligned}
$$$$
\left.=\llbracket \mathrm{f}(\mathrm{fn}() \Rightarrow \text { eval } \mathrm{m} 2 \mathrm{p}) \rrbracket^{\mathrm{ml}} \xi^{\prime \prime}\left[\mathrm{f} \mapsto\left(\lambda t \cdot i_{D S}\left(f\left(i_{D S}^{-1}(t *)\right)\right)\right)\right]\right)
$$$$
=\left(\lambda t . i_{D S}\left(f\left(i_{D S}^{-1}(t *)\right)\right)\right)\left(\lambda u . \theta_{\text {eval }} m_{1} \star \lambda g . g \varrho\right)
$$$$
=\left(\lambda t . i_{D S}\left(f\left(i_{D S}^{-1}(t *)\right)\right)\right)\left(\lambda u . i_{D S}\left(\llbracket m_{2} \rrbracket_{\mathrm{r}} \rho\right)\right)
$$$$
=i_{D S}\left(f\left(\llbracket m_{2} \rrbracket_{\mathrm{r}} \rho\right)\right)
$$

(By IH on $m_{2}$ )

Similarly,

$$
\begin{aligned}
& i_{D S}\left(\llbracket m \rrbracket_{\mathrm{r}} \rho\right) \\
& =i_{D S}\left(\llbracket \operatorname{APP}\left(m_{1}, m_{2}\right) \rrbracket_{\mathrm{r}} \rho\right) \\
& =i_{D S}\left(\psi_{\mathrm{r}}(\text { fun }(f))\left(\llbracket m_{2} \rrbracket_{\mathrm{r}} \rho\right)\right) \\
& =i_{D S}\left(f\left(\llbracket m_{2} \rrbracket_{\mathrm{r}} \rho\right)\right)
\end{aligned}
$$

Lemma 15 For all $m \in \Lambda$, $\operatorname{norm}(m)=\theta_{\text {norm }} m$.

Proof: $\quad$ Let $m$ be given, and let $\xi=\emptyset\left[\right.$ down $\mapsto \theta_{\text {down }}$, eval $\mapsto \theta_{\text {eval }}$, norm $\mapsto \theta_{\text {norm }}, \mathrm{m} \mapsto$ $m]$. Let further $\lfloor\varrho\rfloor=\llbracket$ fn $\mathrm{x}=>\operatorname{TM}(\mathrm{fn} \mathrm{n}=>\operatorname{VAR}(\mathrm{x})) \rrbracket^{\mathrm{ml}} \xi$ and $\rho=\left(\lambda x^{\mathrm{V}} \cdot \uparrow(\widehat{\operatorname{VAR}}(x))\right)$. 
We first verify that $\varrho$ and $\rho$ satisfy the requirements of Lemma 14 , namely that for all $x^{\prime} \in \mathrm{V} \supset F V(m)$,

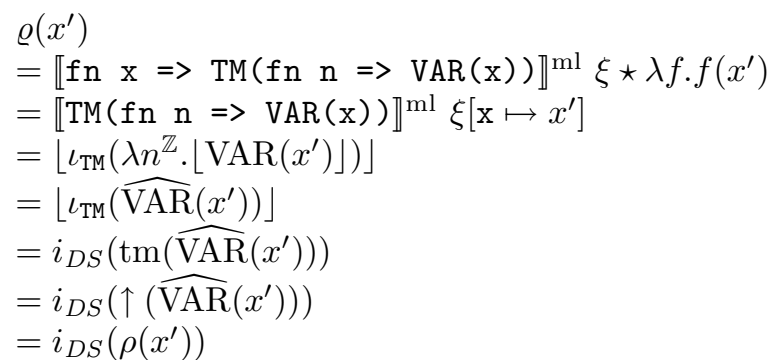

Hence, by a single unrolling of the fixed-point equation $\theta_{\text {norm }}=\Theta_{\text {norm }}\left(\theta_{\text {norm }}\right)$,

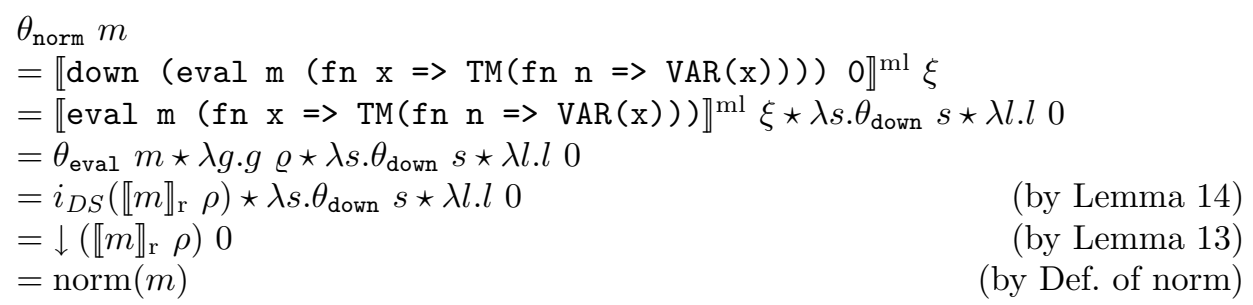

Theorem 4 (implementation correctness) The program NORM satisfies that for all $m, m^{\prime} \in \Lambda, N O R M \bullet(m)=m^{\prime} \Leftrightarrow \operatorname{norm}(m)=\left\lfloor m^{\prime}\right\rfloor$.

Proof: A direct consequence of Lemma 15 and Theorem 3, since $\llbracket N O R M \rrbracket^{\mathrm{ml}} \emptyset=$ $\left\lfloor\theta_{\text {norm }}\right\rfloor$.

Together with semantic correctness (Theorem 2) and Definition 2 of norm, this tells us that NORM correctly computes the normal form of all $\lambda$-terms without free occurrences of $\mathrm{g} \underline{i}$-variables (including, in particular, all closed terms).

\section{A generalization to Böhm trees}

Recall that the correctness of our normalization algorithm was expressed in terms of simple conditionals. Soundness was, essentially, "if the algorithm returns a result, that result is correct"; and completeness, "if a correct result exists, the algorithm will find one". We now set out to extend these statements to a more general notion of normal forms, effectively replacing "if" with "to the extent that".

\subsection{From $\lambda$-terms to $\lambda$-trees}

We adopt a new view of normalization results, generalizing the flat domain $\Lambda_{\perp}$ of lifted $\lambda$-terms to a more elaborate domain of lazy $\lambda$-trees, which will allow us to talk formally about partial and infinite terms. The intended reading of a $\lambda$-tree result is that the finitely reachable, defined parts of the tree represent committed output from the normalizer. 
Syntax Let $\underline{\Lambda}$ be the cpo of $\lambda$-trees $M$, defined as a minimal-invariant solution to the recursive domain equation

$$
i_{\underline{\Lambda}}: \underline{\Lambda} \stackrel{\cong}{\Rightarrow}(\mathrm{V}+\mathrm{V} \times \underline{\Lambda}+\underline{\Lambda} \times \underline{\Lambda})_{\perp},
$$

with the constructors for $\underline{\Lambda}$-elements given by:

$$
\begin{aligned}
\square & =i_{\underline{\Lambda}}^{-1}(\perp) & \underline{\operatorname{LAM}}\left(x, M_{0}\right) & =i_{\underline{\Lambda}}^{-1}\left(\left\lfloor i n_{2}\left(x, M_{0}\right)\right\rfloor\right) \\
\underline{\operatorname{VAR}}(x) & =i_{\underline{\Lambda}}^{-1}\left(\left\lfloor i n_{1}(x)\right\rfloor\right) & \underline{\operatorname{APP}}\left(M_{1}, M_{2}\right) & =i_{\underline{\Lambda}}^{-1}\left(\left\lfloor i n_{3}\left(M_{1}, M_{2}\right)\right\rfloor\right)
\end{aligned}
$$

Again, any element of $\underline{\Lambda}$ can be uniquely written as one of these four forms.

We also have a natural interpretation of the domain-theoretic ordering on $\underline{\Lambda}$ : $M \sqsubseteq$ $M^{\prime}$ precisely when $M^{\prime}$ can be obtained by replacing some occurrences of $\square$ in $M$ with suitable subtrees. Note that, since $\underline{\Lambda}$ is a cpo, it necessarily also contains infinite trees, such as $\bigsqcup_{n \in \omega} \underline{\operatorname{LAM}}\left(\mathrm{x}_{1}, \cdots \underline{\operatorname{LAM}}\left(\mathrm{x}_{n}, \square\right)\right)=\underline{\operatorname{LAM}}\left(\mathrm{x}_{1}, \underline{\operatorname{LAM}}\left(\mathrm{x}_{2}, \cdots\right)\right)$.

We define the cut function $|\cdot| \cdot: \underline{\Lambda} \times \omega \rightarrow \underline{\Lambda}$ by induction on $k$ :

$$
\begin{aligned}
& |M|^{0}=\square \quad|\square|^{k+1}=\square \quad|\underline{\operatorname{VAR}}(x)|^{k+1}=\underline{\operatorname{VAR}}(x) \\
& \left|\underline{\operatorname{LAM}}\left(x, M_{0}\right)\right|^{k+1}=\underline{\operatorname{LAM}}\left(x,\left|M_{0}\right|^{k}\right) \quad\left|\underline{\operatorname{APP}}\left(M_{1}, M_{2}\right)\right|^{k+1}=\underline{\operatorname{APP}}\left(\left|M_{1}\right|^{k},\left|M_{2}\right|^{k}\right)
\end{aligned}
$$

That is, $|M|^{k}$ replaces all parts of the tree $M$ above height $k$ with $\square$. As we would expect, every tree is the limit of its finite cuts:

Lemma 16 For any $M \in \underline{\Lambda}, M=\bigsqcup_{k \in \omega}|M|^{k}$.

Proof: The function $\delta:[\underline{\Lambda} \rightarrow \underline{\Lambda}] \rightarrow[\underline{\Lambda} \rightarrow \underline{\Lambda}]$ associated to the domain equation is given by

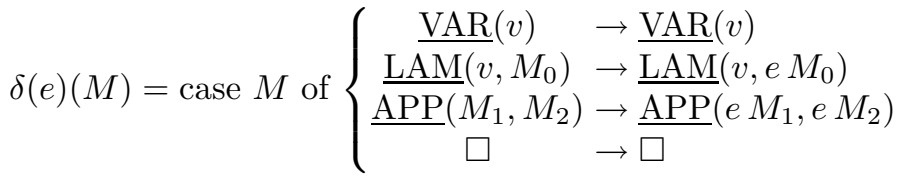

It is easy to check, by induction on $k$, that $|M|^{k}=\delta^{k}\left(\perp_{[\underline{\Lambda} \rightarrow \underline{\Lambda}]}\right)(M)$. Hence, by the minimal-invariant property, $\bigsqcup_{k \in \omega}|M|^{k}=\bigsqcup_{k \in \omega} \delta^{k}(\perp) M=\left(\bigsqcup_{k \in \omega} \delta^{k}(\perp)\right) M=$ $\operatorname{fix}(\delta)(M)=i d_{\underline{\Lambda}} M=M$.

A tree is called finite if it is equal to one of its cuts, and total if it contains no $\square$. Thus, finite, total $\lambda$-trees are in one-to-one correspondence with ordinary $\lambda$-terms, as previously defined. We also have a natural inclusion of ordinary $\lambda$-terms into $\lambda$-trees, $\langle\cdot\rangle: \Lambda \rightarrow \underline{\Lambda}$, defined inductively in the obvious way.

Compatibility We can extend any predicate on $\lambda$-terms, $P \subseteq \Lambda$, to a corresponding predicate on $\lambda$-trees, $P^{\dagger} \subseteq \underline{\Lambda}$, by

$$
P^{\dagger}=\left\{\left.M \in \underline{\Lambda}|\forall k \in \omega . \exists m \in P .| M\right|^{k} \sqsubseteq\langle m\rangle\right\} .
$$

That is, $M \in P^{\dagger}$ if every finite cut of $M$ can be increased to a total tree, satisfying the original predicate. When a tree already represents a proper term, the extension has no effect: $\langle m\rangle \in P^{\dagger}$ iff $m \in P$. We also note that $P^{\dagger}$ is downward closed: if $M \in P^{\dagger}$ and $M^{\prime} \sqsubseteq M$, then also $M^{\prime} \in P^{\dagger}$; and that extension is monotone: if $P \subseteq P^{\prime}$, then $P^{\dagger} \subseteq P^{\prime \dagger}$. 
Definition 5 For $M \in \underline{\Lambda}$ and $m \in \Lambda^{\Delta}$, we define the compatibility relations $\leftrightarrow^{\dagger}$ and $\leftrightarrow{ }_{\Delta}^{\dagger} b y$

$$
\begin{aligned}
M \leftrightarrow^{\dagger} m & \Leftrightarrow M \in\left\{m^{\prime} \in \Lambda \mid m^{\prime} \leftrightarrow m\right\}^{\dagger} \\
M \leftrightarrow_{\Delta}^{\dagger} m & \Leftrightarrow M \in\left\{m^{\prime} \in \Lambda^{\Delta} \mid m^{\prime} \leftrightarrow m\right\}^{\dagger}
\end{aligned}
$$

Note that, like convertibility, compatibility is defined with respect to concrete terms, not $\alpha$-equivalence classes. Thus,

$$
\underline{\operatorname{LAM}}\left(\mathrm{g}_{0}, \underline{\operatorname{LAM}}\left(\mathrm{g}_{0}, \square\right)\right) \leftrightarrow^{\dagger} \operatorname{LAM}(\mathrm{x}, \operatorname{LAM}(\mathrm{y}, \operatorname{VAR}(\mathrm{y})))
$$

(because the $\square$ can still be increased to $\underline{\operatorname{VAR}}\left(\mathrm{g}_{0}\right)$, making the two sides convertible), but we do not have

$$
\underline{\operatorname{LAM}}\left(\mathrm{g}_{0}, \underline{\operatorname{LAM}}\left(\mathrm{g}_{0}, \square\right)\right) \leftrightarrow^{\dagger} \operatorname{LAM}(\mathrm{x}, \operatorname{LAM}(\mathrm{y}, \operatorname{VAR}(\mathrm{x}))) .
$$

Böhm trees We can view Böhm trees [Bar84, Chapter 10] as a particular kind of $\lambda$-trees. Informally, a Böhm tree is either $\square$, or a generalized head normal form,

$$
\underline{\operatorname{LAM}}\left(x_{1}, \cdots \underline{\operatorname{LAM}}\left(x_{n}, \underline{\operatorname{APP}}\left(\underline{\operatorname{APP}}\left(\underline{\operatorname{VAR}}(x), M_{1}\right), \cdots M_{m}\right)\right)\right),
$$

where $n, m \geq 0$, and each $M_{i}$ is itself a Böhm tree. However, we need to make precise how this evidently circular definition is to be interpreted.

Formally, we define Böhm trees in terms of the following rules:

$$
\begin{aligned}
& \overline{\vdash_{\text {bt }} \square}(\mathrm{BT}-\square) \quad \frac{\vdash_{\text {at }} M}{\vdash_{\text {nf }} M} \text { (NF-AT) } \quad \frac{}{\vdash_{\text {at }} \underline{\operatorname{VAR}(x)}} \text { (AT-VAR) } \\
& \frac{\vdash_{\mathrm{nf}} M}{\vdash_{\mathrm{bt}} M} \text { (BT-NF) } \frac{\vdash_{\mathrm{nf}} M_{0}}{\vdash_{\mathrm{nf}} \underline{\operatorname{LAM}}\left(x, M_{0}\right)} \text { (NF-LAM) } \frac{\vdash_{\mathrm{at}} M_{1} \quad \vdash_{\mathrm{bt}} M_{2}}{\vdash_{\mathrm{at}} \underline{\operatorname{APP}\left(M_{1}, M_{2}\right)}} \text { (AT-APP) }
\end{aligned}
$$

These rules determine an operator $F$ on subsets of $B=\{\mathrm{bt}$, nf, at $\} \times \underline{\Lambda}$, where $F(X)$ is the set of conclusions occurring in rule instances with premises from $X$ :

$$
F(X)=\{(\mathrm{bt}, \square)\} \cup \cdots \cup\left\{\left(\mathrm{at}, \underline{\operatorname{APP}}\left(M_{1}, M_{2}\right)\right) \mid\left(\text { at }, M_{1}\right) \in X \wedge\left(\mathrm{bt}, M_{2}\right) \in X\right\} .
$$

$F$ is clearly monotone, i.e., $X \subseteq X^{\prime} \Rightarrow F(X) \subseteq F\left(X^{\prime}\right)$. We say that a set $X \subseteq B$ is $F$-closed if $F(X) \subseteq X$, i.e., if everything derivable by the rule instances with premises from $X$, is already in $X$.

When $X$ is inclusive, so is $F(X)$, because inclusiveness is preserved by finite unions, and the constructor functions (as well as pairing with constants) are order-monics, i.e., also reflect $\sqsubseteq$, so their direct images preserve inclusiveness.

Since both subsets of $B$ and inclusive subsets of $B$ are closed under arbitrary intersections, they each form complete lattices. Thus, by the Knaster-Tarski fixedpoint theorem, we get the least $F$-closed set $\mathcal{B}^{\text {fin }} \subseteq B$ by taking the intersection over all $F$-closed subsets of $B$, and the least $F$-closed inclusive set $\mathcal{B}^{\text {inf }} \subseteq B$ as the intersection of all $F$-closed inclusive subsets of $B$.

The associated rule induction principles are: if a predicate $P$ on $B$ is $F$-closed, then $\mathcal{B}^{\text {fin }} \subseteq P$ (since $\mathcal{B}^{\text {fin }}$ was the least $F$-closed set). Analogously, if $P$ is both $F$ closed and inclusive, then $\mathcal{B}^{\inf } \subseteq P$. As special cases we get inversion principles: 
$\mathcal{B}^{\text {fin }}=F\left(\mathcal{B}^{\text {fin }}\right)$ and $\mathcal{B}^{\text {inf }}=F\left(\mathcal{B}^{\text {inf }}\right)$, i.e., every element of either set can be written as the conclusion of a rule with premises in the corresponding set. Naturally, $\mathcal{B}^{\text {fin }} \subseteq \mathcal{B}^{\text {inf }}$, because $\mathcal{B}^{\text {fin }}$ is the least of all fixed points of $F$.

We write $\mathcal{B}_{s}^{\text {fin }}$ for $\left\{M \mid(s, M) \in \mathcal{B}^{\text {fin }}\right\}$, and analogously for $\mathcal{B}_{s}^{\text {inf }}$. The set of Böhm trees is then defined to be $\mathcal{B}_{\mathrm{bt}}^{\text {inf }}$; the finite Böhm trees are $\mathcal{B}_{\mathrm{bt}}^{\text {fin }}$.

(Note, incidentally, that Böhm trees are not simply the uniform extension of finite normal forms to $\lambda$-trees, $\mathcal{N}_{\text {nf }}^{\dagger}$. The latter (which could be called infinitary normal forms) are merely the $\lambda$-trees that do not contain any evident $\beta$-redexes. We thus have $\mathcal{B}_{\mathrm{bt}}^{\inf } \subseteq \mathcal{N}_{\mathrm{nf}}^{\dagger}$, but the opposite inclusion does not hold: infinitary normal forms include non-Böhm trees, such as $\underline{\operatorname{LAM}}(x, \square)$ or $\underline{\operatorname{APP}}(\underline{\operatorname{APP}}(\cdots, \mathrm{x}), \mathrm{x})$.

Nor should $\mathcal{B}_{\mathrm{bt}}^{\text {inf }}$ be confused with the set of trees determined by a coinductive reading of the above rules, i.e., the greatest fixed point of $F$. That set still contains, e.g., the tree $\underline{\operatorname{LAM}}\left(\mathrm{x}_{0}, \underline{\operatorname{LAM}}\left(\mathrm{x}_{1}, \cdots\right)\right)$. Even though we allow Böhm trees to be infinite, each run of curried abstractions or applications must be finite, like in the inductive reading of the rules.)

Again, we expect that a reduction-free Böhm normalizer will output Böhm trees that are compatible with the input term (soundness); that convertible inputs are mapped to the same Böhm tree (identification); and that the output tree is as large as possible (completeness).

\subsection{A semantic Böhm-tree construction}

The modularity of output-term generation, originally motivated by flexible generation of fresh names, also allows us to "locally" re-target the existing normalization construction to Böhm trees.

Output-tree generation For any $f: A \rightarrow B$, where $A$ and $B$ are pointed cpos, we define its smashed strict extension, $\bullet \circledast f: A \rightarrow B$, by $\perp_{A} \circledast f=\perp_{B}$, and $a \circledast f=f(a)$ otherwise.

We first define tree-based analogs of the wrapper functions from Section 2.2, again using de Bruijn-level naming:

Definition 6 (cf. Definition 1) Let $\widehat{\Lambda}=[\mathrm{N} \rightarrow \underline{\Lambda}]$, and define

$$
\begin{aligned}
\widehat{\operatorname{VAR}}(v) & =\lambda n^{\mathrm{N}} \cdot \underline{\operatorname{VAR}}(v) \\
\widehat{\operatorname{LAM}}(f) & =\lambda n^{\mathrm{N}} \cdot f(\operatorname{gen}(n))(n+1) \circledast \lambda M_{0}^{\underline{\Lambda}} \cdot \underline{\operatorname{LAM}}\left(\operatorname{gen}(n), M_{0}\right) \\
\widehat{\operatorname{APP}}\left(l_{1}, l_{2}\right) & =\lambda n^{\mathrm{N}} \cdot l_{1} n \circledast \lambda M_{1}^{\Lambda} \cdot \underline{\operatorname{APP}}\left(M_{1}, l_{2} n\right)
\end{aligned}
$$

Note that $\widehat{\mathrm{APP}}$ is strict in its first argument only. Making it strict in both, would revert the normalizer to always produce either $\square$ or a finite, total $\lambda$-tree as the result, just like the original version. Strictness in the first argument does not actually matter, since the function will never be applied to a $\square$-representative; however, from an operational viewpoint, it is convenient to know that it is safe to evaluate the argument eagerly. Making $\widehat{\mathrm{LAM}}$ non-strict would not affect correctness with respect to compatibility, but the output would no longer necessarily be a Böhm tree. 
The residualizing model The construction of the residualizing model from Section 2.3 can be reused verbatim, since it only relies on $\widehat{\Lambda}$ being a pointed cpo with continuous wrapper functions. Only the codomain of the putative Böhm normalizer changes:

Definition 7 (cf. Definition 2) For any $\Delta$, we define the function bt $\mathrm{bt}_{\Delta} \Lambda^{\Delta} \rightarrow \underline{\Lambda}$ by

$$
\mathrm{bt}_{\Delta}(m)=\downarrow\left(\llbracket m \rrbracket_{\mathrm{r}}\left(\lambda x^{\mathrm{V}} \cdot \uparrow(\widehat{\operatorname{VAR}}(x))\right)\right) \sharp \Delta .
$$

Again, we write just bt : $\Lambda \rightarrow \underline{\Lambda}$ for the variant where $\sharp \Delta$ is replaced with 0 , noting that it agrees with $\mathrm{bt}_{\Delta}$ whenever $\Delta \cap \mathrm{G}=\emptyset$.

\subsection{Correctness of the construction}

The proof proceeds very much like in the original, finitary case.

\section{Basic results about compatibility}

Lemma $17 P^{\dagger}$ is inclusive for any $P$.

Proof: We need to show that $\forall k \in \omega . \exists m \in P .|M|^{k} \sqsubseteq\langle m\rangle$ is an inclusive predicate in $M$. By closure under intersections, it is enough to consider a fixed $k$. But, for any chain $\left(M_{i}\right)_{i \in \omega}$, there must be an $i_{0}$ such that $\forall i \geq i_{0} \cdot\left|M_{i}\right|^{k}=\left|M_{i_{0}}\right|^{k}$. Hence, if $M_{i_{0}} \in P^{\dagger}$, the $m \in P$ such that $\left|M_{i_{0}}\right|^{k} \sqsubseteq\langle m\rangle$ will also work for all subsequent $i$, and thus also for $\bigsqcup_{i \in \omega} M_{i}$.

Lemma 18 The constructor functions preserve compatibility:

a. For all $m$ and $\Delta, \square \leftrightarrow{ }_{\Delta}^{\dagger} m$.

b. If $v \in \Delta$, then $\underline{\operatorname{VAR}}(v) \leftrightarrow_{\Delta}^{\dagger} \operatorname{VAR}(v)$.

c. If $M \leftrightarrow_{\Delta \cup\{v\}}^{\dagger} m$, then $\underline{\operatorname{LAM}}(v, M) \leftrightarrow_{\Delta}^{\dagger} \operatorname{LAM}(v, m)$.

d. If $M_{1} \leftrightarrow{ }_{\Delta}^{\dagger} m_{1}$ and $M_{2} \leftrightarrow_{\Delta}^{\dagger} m_{2}$, then $\underline{\operatorname{APP}}\left(M_{1}, M_{2}\right) \leftrightarrow_{\Delta}^{\dagger} \operatorname{APP}\left(m_{1}, m_{2}\right)$.

Proof: Straightforward. Part (a) uses that $\square$ is the least element in $\underline{\Lambda}$ and that $\leftrightarrow$ is reflexive; part (b) uses that both $\sqsubseteq$ and $\leftrightarrow$ are reflexive; parts (c) and (d) use that $\underline{\operatorname{LAM}}(v, \cdot)$ and $\underline{\operatorname{APP}}(\cdot, \cdot)$ are monotonic and that $\leftrightarrow$ is a congruence wrt. LAM and $\mathrm{APP}$, respectively.

\section{Correctness of the wrappers}

Definition 8 (cf. Definition 3) For $l \in \widehat{\Lambda}, m \in \Lambda^{\Delta}$, and $s \in\{$ nf, at $\}$, we define the representation relation $\lesssim b y$

$$
l \lesssim_{s}^{\Delta} m \text { iff } \forall n \geq \sharp \Delta . l n \leftrightarrow{ }_{\Delta}^{\dagger} m \wedge\left(l n=\square \vee \ln \in \mathcal{B}_{s}^{\text {inf }}\right) .
$$

The correctness of the wrappers will need to be established with respect to the new definition of $\lesssim$. The original "interface" lemmas of $\lesssim(L e m m a s ~ 3,4$, and 5 ) can actually be restated verbatim - this considerably simplifies establishing soundness. Of course, the underlying meanings, and hence the proofs, of the Lemmas do change, according to the new definitions for the Böhm-tree construction. 
Lemma 19 (cf. Lemma 3) For fixed $\Delta$, $s$, and $m$, the predicate $P=\left\{l \mid l \lesssim_{s}^{\Delta} m\right\}$ is pointed and inclusive.

Proof: Pointedness is immediate. Inclusiveness also follows directly, since the relation is defined in terms of universal quantification, conjunction, finite disjunction, and inverse image by the (continuous) application function from the inclusive predicates $\leftrightarrow_{\Delta}^{\dagger}$ (by Definition 5 and Lemma 17 ) and $\mathcal{B}_{s}^{\text {inf }}$ (by construction).

Lemma 20 (cf. Lemma 4) The representation relation is closed under weakening and conversion:

a. If $l \lesssim_{s}^{\Delta} m$ and $\Delta \subseteq \Delta^{\prime}$, then also $l \lesssim_{\Delta^{\prime}}^{\prime} m$.

b. If $l \lesssim_{s}^{\Delta} m$ and $m^{\prime} \in \Lambda^{\Delta}$ with $m \leftrightarrow m^{\prime}$, then also $l \lesssim_{s}^{\Delta} m^{\prime}$.

Proof: The proof is analogous to that of Lemma 4, with part (a) now exploiting monotonicity of predicate extension: when $n \geq \sharp \Delta^{\prime} \geq \sharp \Delta, l n \in\left\{m^{\prime} \in \Lambda^{\Delta} \mid m^{\prime} \leftrightarrow\right.$ $m\}^{\dagger} \subseteq\left\{m^{\prime} \in \Lambda^{\Delta^{\prime}} \mid m^{\prime} \leftrightarrow m\right\}^{\dagger}$. Part (b) just uses transitivity of $\leftrightarrow$, like in the original proof.

Lemma 21 (cf. Lemma 5) Representations of terms behave much like the terms themselves:

a. If $v \in \Delta$, then $\widehat{\operatorname{VAR}}(v) \lesssim \Delta$ at $\operatorname{VAR}(v)$.

b. If $l_{1} \lesssim \Delta m_{1}$ and $l_{2} \lesssim_{\mathrm{nf}}^{\Delta} m_{2}$, then $\widehat{\operatorname{APP}}\left(l_{1}, l_{2}\right) \lesssim_{\text {at }}^{\Delta} \operatorname{APP}\left(m_{1}, m_{2}\right)$.

c. If $l \lesssim \Delta$ at $m$, then also $l \lesssim \Delta$ nf $m$.

d. Let $f \in[\mathrm{V} \rightarrow \widehat{\Lambda}]$ and $m \in \Lambda^{\Delta \cup\{x\}}$. If $\forall v \notin \Delta . f v \lesssim_{\mathrm{nf}}^{\Delta \cup\{v\}} m[\operatorname{VAR}(v) / x]$, then $\widehat{\operatorname{LAM}}(f) \lesssim_{\mathrm{nf}}^{\Delta} \operatorname{LAM}(x, m)$.

Proof: In each case, assume that an arbitrary $n \geq \sharp \Delta$ is given. Then the proofs for each case proceed as follows:

a. Let arbitrary $v \in \Delta$ be given. Then $\widehat{\operatorname{VAR}}(v) n=\underline{\operatorname{VAR}}(v)$. The first conjunct in $\lesssim$ then follows from Lemma $18(\mathrm{~b})$, and the second by Rule AT-VAR.

b. Assume that $l_{1} \lesssim_{\Delta}^{\Delta} m_{1}$ and $l_{2} \lesssim_{\text {nf }}^{\Delta} m_{2}$. Let further $M_{1}=l_{1} n$ and $M_{2}=l_{2} n$. Consider $M_{1}$. If $M_{1}=\square$, then $\widehat{\operatorname{APP}}\left(l_{1}, l_{2}\right) n=\square$; thus, the first conjunct holds by Lemma 18(a), and the second by its left disjunct.

If $M_{1} \neq \square$, then $\widehat{\operatorname{APP}}\left(l_{1}, l_{2}\right) n=\underline{\operatorname{APP}}\left(M_{1}, M_{2}\right)$. By definition of $\lesssim$ for $l_{1}$, $M_{1} \leftrightarrow{ }_{\Delta}^{\dagger} m_{1}$ and $M_{1} \in \mathcal{B}_{\text {at }}^{\text {inf }}$. Analogously, $M_{2} \leftrightarrow_{\Delta}^{\dagger} m_{2}$ and $M_{2}=\square \vee M_{2} \in$

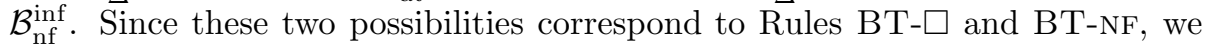
have $M_{2} \in \mathcal{B}_{\mathrm{bt}}^{\text {inf }}$. And thus, $\underline{\operatorname{APP}}\left(M_{1}, M_{2}\right) \leftrightarrow_{\Delta}^{\dagger} \operatorname{APP}\left(m_{1}, m_{2}\right)$ follows from Lemma $18(\mathrm{~d})$, and $\underline{\operatorname{APP}}\left(M_{1}, M_{2}\right) \in \mathcal{B}_{\text {at }}^{\text {inf }}$ by Rule AT-APP.

c. Immediate, by Rule NF-AT. 
d. Let $f, x$, and $m$, satisfy the condition of the lemma, and let $M_{0}=f \mathrm{~g}_{n}(n+$ 1). Consider $M_{0}$. If $M_{0}=\square$, then $\widehat{\operatorname{LAM}}(f) n=\square$, so the first conjunct of $\widehat{\operatorname{LAM}}(f) \lesssim_{\text {nf }}^{\Delta} \operatorname{LAM}(x, m)$ holds by Lemma $18(\mathrm{a})$, and the second by its left disjunct.

If $M_{0} \neq \square$, then $\widehat{\operatorname{LAM}}(f) n=\underline{\operatorname{LAM}}\left(\mathrm{g}_{n}, M_{0}\right)$. By definition of $\sharp \Delta, \mathrm{g}_{n} \notin \Delta$, and so by assumption on $f, f \mathrm{~g}_{n} \underset{\mathrm{nf}^{\Delta \cup}}{\left.\stackrel{\mathrm{g}}{\mathrm{g}} \mathrm{g}_{n}\right\}} m\left[\operatorname{VAR}\left(\mathrm{g}_{n}\right) / x\right]$. Thus, since $n+1 \geq$ $\sharp\left(\Delta \cup\left\{\mathrm{g}_{n}\right\}\right)$, the definition of $\lesssim$ gives us that

$$
M_{0} \leftrightarrow_{\Delta \cup\left\{\mathrm{g}_{n}\right\}}^{\dagger} m\left[\operatorname{VAR}\left(\mathrm{g}_{n}\right) / x\right] \wedge M_{0} \in \mathcal{B}_{\mathrm{nf}}^{\mathrm{inf}} .
$$

By Lemma 18(c), $\underline{\operatorname{LAM}}\left(\mathrm{g}_{n}, M_{0}\right) \leftrightarrow_{\Delta}^{\dagger} \operatorname{LAM}\left(\mathrm{g}_{n}, m\left[\operatorname{VAR}\left(\mathrm{g}_{n}\right) / x\right]\right)$. Now, since $\mathrm{g}_{n} \notin$ $\Delta$ ensures that $\mathrm{g}_{n} \notin F V(m) \backslash\{x\}, \operatorname{LAM}\left(\mathrm{g}_{n}, m\left[\operatorname{VAR}\left(\mathrm{g}_{n}\right) / x\right]\right) \leftrightarrow \operatorname{LAM}(x, m)$ is a valid $\alpha$-conversion. By construction of $\leftrightarrow_{\Delta}^{\dagger}$ (Definition 5) and transitivity of $\leftrightarrow$, also $\underline{\operatorname{LAM}}\left(\mathrm{g}_{n}, M_{0}\right) \leftrightarrow_{\Delta}^{\dagger} \operatorname{LAM}(x, m)$. Finally, from $M_{0} \in \mathcal{B}_{\mathrm{nf}}^{\text {inf }}$, we get $\underline{\operatorname{LAM}}\left(\mathrm{g}_{n}, M_{0}\right) \in \mathcal{B}_{\mathrm{nf}}^{\text {inf }}$ by Rule NF-LAM.

Adequacy of the residualizing model By virtue of the above "interface" lemmas, the verbatim insertion of Lemmas $6,7,8$, and 9 and their proofs remain correct with the Böhm tree definitions, modulo a simple substitution of references to Lemmas 3-5 with references to Lemmas 19-21, respectively.

Correctness of the Böhm-tree normalization function The key technical property of Böhm trees we will need for the completeness result, is that any finite cut of a Böhm tree can be extended to a finite Böhm tree, still approximating the original one. Thus, it will suffice to consider only approximants of the output term that are themselves Böhm trees.

Definition 9 For any Böhm tree $M \in \mathcal{B}_{\mathrm{bt}}^{\text {inf }}$, and $k \in \omega$, we define the Böhm cut $\|M\|^{k}$ by induction on $k$, as follows:

$$
\begin{gathered}
\|M\|^{0}=\square \quad\|\square\|^{k+1}=\square \quad\|\underline{\operatorname{VAR}}(x)\|^{k+1}=\underline{\operatorname{VAR}}(x) \\
\left\|\underline{\operatorname{LAM}}\left(x, M_{0}\right)\right\|^{k+1}=\left\|M_{0}\right\|^{k} \circledast \lambda M_{0}^{\prime} \cdot \underline{\operatorname{LAM}}\left(x, M_{0}^{\prime}\right) \\
\left\|\underline{\operatorname{APP}}\left(M_{1}, M_{2}\right)\right\|^{k+1}=\left\|M_{1}\right\|^{k} \circledast \lambda M_{1}^{\prime} \cdot \underline{\operatorname{APP}}\left(M_{1}^{\prime},\left\|M_{2}\right\|^{k}\right)
\end{gathered}
$$

Intuitively, $\|M\|^{k}$ is the largest (necessarily finite) Böhm tree such that $\|M\|^{k} \sqsubseteq|M|^{k}$. It is constructed by cutting off branches early, if they do not reach a complete Böhmsubtree within the remaining height limit.

Lemma 22 Böhm cuts satisfy:

a. $\forall k \cdot\|M\|^{k} \in \mathcal{B}_{\mathrm{bt}}^{\mathrm{fin}}$.

b. $\forall k \cdot\|M\|^{k} \sqsubseteq|M|^{k}$.

c. $\forall k . \exists k^{\prime} .|M|^{k} \sqsubseteq\|M\|^{k^{\prime}}$.

(These are the only properties of $\|M\|^{k}$ that we will subsequently use.) 
Proof: We prove $(a)$ and $(b)$ together by, induction on $k$. (They could also have been proven separately). The strengthened induction hypothesis is

$$
P(k) \Leftrightarrow \forall(s, M) \in \mathcal{B}^{\text {inf }} .\left(\|M\|^{k} \in \mathcal{B}_{s}^{\text {fin }} \vee\|M\|^{k}=\square\right) \wedge\|M\|^{k} \sqsubseteq|M|^{k} .
$$

All parts of $P(0)$ are immediate. For the inductive step, we assume $P(k)$ and aim to show $P(k+1)$. Let $M \in \mathcal{B}_{s}^{\text {inf }}$; we distinguish cases on $M$ :

Case $M=\square$ : All parts are trivial.

Case $M=\underline{\operatorname{VAR}}(x)$ : Then $\|M\|^{k+1}=\underline{\operatorname{VAR}}(x)$, which belongs to all of $\mathcal{B}_{\mathrm{at}}^{\mathrm{fin}}, \mathcal{B}_{\mathrm{nf}}^{\mathrm{fin}}$, and $\mathcal{B}_{\mathrm{bt}}^{\mathrm{fin}} ;$ and $\underline{\operatorname{VAR}}(x)=|\underline{\operatorname{VAR}}(x)|^{k+1}$.

Case $M=\underline{\operatorname{LAM}}\left(x, M_{0}\right)$ : By the inversion principle for $\mathcal{B}^{\text {inf }}, M \in \mathcal{B}_{\mathrm{bt}}^{\mathrm{inf}}$ must be because $M \in \mathcal{B}_{\mathrm{nf}}^{\text {inf }}$ and that again must be because $M_{0} \in \mathcal{B}_{\mathrm{nf}}^{\mathrm{inf}}$. Now consider $\left\|M_{0}\right\|^{k}$. If $\left\|M_{0}\right\|^{k}=\square$, then also $\|M\|^{k+1}=\square$, and all requirements are trivially satisfied. Otherwise, $\|M\|^{k+1}=\underline{\operatorname{LAM}}\left(x,\left\|M_{0}\right\|^{k}\right)$. By the IH, we get that $\left\|M_{0}\right\|^{k} \in \mathcal{B}_{\mathrm{nf}}^{\mathrm{fin}}$ and $\left\|M_{0}\right\|^{k} \sqsubseteq\left|M_{0}\right|^{k}$, from which we immediately conclude that also $\underline{\operatorname{LAM}}\left(x,\left\|M_{0}\right\|^{k}\right) \in \mathcal{B}_{\text {nf }}^{\text {fin }}$ and $\underline{\operatorname{LAM}}\left(x,\left\|M_{0}\right\|^{k}\right) \sqsubseteq \underline{\operatorname{LAM}}\left(x,\left|M_{0}\right|^{k}\right)=|M|^{k+1}$.

Case $M=\underline{\operatorname{APP}}\left(M_{1}, M_{2}\right)$ : Like above, we must have $\underline{\operatorname{APP}}\left(M_{1}, M_{2}\right) \in \mathcal{B}_{\text {at }}^{\text {inf }} \subseteq \mathcal{B}_{\mathrm{nf}}^{\text {inf }} \subseteq$ $\mathcal{B}_{\mathrm{bt}}^{\text {inf }}$, hence $M_{1} \in \mathcal{B}_{\mathrm{at}}^{\text {inf }}$ and $M_{2} \in \mathcal{B}_{\mathrm{bt}}^{\text {inf }}$. If $\left\|M_{1}\right\|^{k}=\bar{\square}$, then also $\|M\|^{k+1}=\square$, and we are done. Otherwise, $\|M\|^{k+1}=\underline{\operatorname{APP}}\left(\left\|M_{1}\right\|^{k},\left\|M_{2}\right\|^{k}\right)$. By IH on $M_{1}$, we get that $\left\|M_{1}\right\|^{k} \in \mathcal{B}_{\text {at }}^{\text {fin }}$ and $\left\|M_{1}\right\|^{k} \sqsubseteq\left|M_{1}\right|^{k}$. By IH on $M_{2},\left\|M_{2}\right\|^{k} \in \mathcal{B}_{\text {bt }}^{\text {fin }}$ (which holds even if $\left.\left\|M_{2}\right\|^{k}=\square\right)$, and $\left\|M_{2}\right\|^{k} \sqsubseteq\left|M_{2}\right|^{k}$. Hence, $\underline{\operatorname{APP}}\left(\left\|M_{1}\right\|^{k},\left\|M_{2}\right\|^{k}\right) \in \mathcal{B}_{\text {at }}^{\text {fin }}$, and $\|M\|^{k+1} \sqsubseteq \underline{\operatorname{APP}}\left(\left|M_{1}\right|^{k},\left|M_{2}\right|^{k}\right)=|M|^{k+1}$.

For (c), the proof is by the inclusive variant of rule induction. We strengthen the induction hypothesis to

$$
P(s, M) \Leftrightarrow \forall k . \exists k^{\prime} .|M|^{k} \sqsubseteq\|M\|^{k^{\prime}} \wedge\left(s \neq \mathrm{bt} \Rightarrow\|M\|^{k^{\prime}} \neq \square\right) .
$$

And we want to show that for all $(s, M) \in \mathcal{B}^{\text {inf }}, P(s, M)$. First, we must establish that $P$ is inclusive. By closedness under intersection, and inverse image by projections, it suffices to show that, for every fixed $s$ and $k$,

$$
P_{s, k}(M) \Leftrightarrow \exists k^{\prime} .|M|^{k} \sqsubseteq\|M\|^{k^{\prime}} \wedge\left(s \neq \mathrm{bt} \Rightarrow\|M\|^{k^{\prime}} \neq \square\right)
$$

is inclusive. So, consider a chain $M_{1} \sqsubseteq M_{2} \sqsubseteq \cdots$ such that there exist $k_{1}^{\prime}, k_{2}^{\prime}, \ldots$ with

$$
\forall i .\left|M_{i}\right|^{k} \sqsubseteq\left\|M_{i}\right\|^{k_{i}^{\prime}} \wedge\left(s \neq \mathrm{bt} \Rightarrow\left\|M_{i}\right\|^{k_{i}^{\prime}} \neq \square\right) ;
$$

we must show that there also exists a $k^{\prime}$ such that $\left.\bigsqcup_{i} M_{i}\right|^{k} \sqsubseteq\left\|\bigsqcup_{i} M_{i}\right\|^{k^{\prime}}$ and $(s \neq$ $\left.\mathrm{bt} \Rightarrow\left\|\bigsqcup_{i} M_{i}\right\|^{k^{\prime}} \neq \square\right)$.

But since there can be no infinite ascending chain of $\lambda$-trees of height at most $k$, there must exist an $i_{0}$ such that $\forall i \geq i_{0},\left|M_{i}\right|^{k}=\left|M_{i_{0}}\right|^{k}$, and so $\|\left.\bigsqcup_{i} M_{i}\right|^{k}=\left|M_{i_{0}}\right|^{k}$. Take $k^{\prime}=k_{i_{0}}^{\prime}$. Then, by monotonicity of $\|-\|^{k^{\prime}}$, we conclude,

$$
\left.\bigsqcup_{i} M_{i}\right|^{k}=\left|M_{i_{0}}\right|^{k} \sqsubseteq\left\|M_{i_{0}}\right\|^{k^{\prime}} \sqsubseteq\left\|\bigsqcup_{i} M_{i}\right\|^{k^{\prime}}
$$

as required. Moreover, when $s \neq \mathrm{bt}$, we have $\left\|M_{i}\right\|^{k^{\prime}} \neq \square$, so also $\left\|\bigsqcup_{i} M_{i}\right\|^{k^{\prime}} \neq \square$.

Now, we need to show that $P$ is closed under the rules defining $\mathcal{B}^{\text {inf }}$. There are 6 cases to consider: 
Case (BT- $\square$ ). To show: $P\left(\right.$ bt, $\square$ ). For any $k,|\square|^{k}=\square$, so we can just take $k^{\prime}=0$. The second conjunct is vacuously true.

Case (BT-nf. To show: $P(\mathrm{bt}, M)$, assuming $P(\mathrm{nf}, M)$. The result follows immediately from the assumption (forgetting the extra guarantee that $\|M\|^{k^{\prime}} \neq \square$ ).

Case (NF-AT). To show: $P(\mathrm{nf}, M)$, assuming $P($ at, $M)$. The result follows immediately from the assumption.

Case (NF-LAM). To show: $P\left(\mathrm{nf}, \underline{\operatorname{LAM}}\left(x, M_{0}\right)\right)$, assuming $P\left(\mathrm{nf}, M_{0}\right)$. Let $k$ be given; we must find a $k^{\prime}$ such that $\left|\underline{\operatorname{LAM}}\left(x, M_{0}\right)\right|^{k} \sqsubseteq\left\|\underline{\operatorname{LAM}}\left(x, M_{0}\right)\right\|^{k^{\prime}}$, and $\left\|\underline{\operatorname{LAM}}\left(x, M_{0}\right)\right\|^{k^{\prime}}$ $\neq \square$.

By the assumption, there exists a $k_{0}^{\prime}$ such that $\left|M_{0}\right|^{k} \sqsubseteq\left\|M_{0}\right\|^{k_{0}^{\prime}}$ and $\left\|M_{0}\right\|^{k_{0}^{\prime}} \neq \square$. Take $k^{\prime}=k_{0}^{\prime}+1$. Then

$$
\begin{aligned}
& \left|\underline{\operatorname{LAM}}\left(x, M_{0}\right)\right|^{k} \sqsubseteq\left|\underline{\operatorname{LAM}}\left(x, M_{0}\right)\right|^{k+1}=\underline{\operatorname{LAM}}\left(x,\left|M_{0}\right|^{k}\right) \sqsubseteq \underline{\operatorname{LAM}}\left(x,\left\|M_{0}\right\|^{k_{0}^{\prime}}\right) \\
& =\left\|M_{0}\right\|^{k_{0}^{\prime}} \otimes \lambda M_{0}^{\prime} \cdot \underline{\underline{\operatorname{LAM}}}\left(x, M_{0}^{\prime}\right)=\left\|\underline{\operatorname{LAM}}\left(x, M_{0}\right)\right\|^{k_{0}^{\prime}+1}=\left\|\underline{\operatorname{LAM}}\left(x, M_{0}\right)\right\|^{k^{\prime}} .
\end{aligned}
$$

Case (AT-VAR). To show: $P($ at, $\underline{\operatorname{VAR}}(x))$. For any $k,|\underline{\operatorname{VAR}}(x)|^{k} \sqsubseteq \underline{\operatorname{VAR}}(x)=$ $\|\underline{\operatorname{VAR}}(x)\|^{1}$, so we can take $k^{\prime}=1$.

Case (AT-APP). To show: $P\left(\right.$ at, $\underline{\operatorname{APP}}\left(M_{1}, M_{2}\right)$, assuming $P\left(\right.$ at,$\left.M_{1}\right)$ and $P\left(\right.$ bt, $\left.M_{2}\right)$. Let $k$ be given; we must find a $k^{\prime}$ such that $\left|\underline{\operatorname{APP}}\left(M_{1}, M_{2}\right)\right|^{k} \sqsubseteq\left\|\underline{\operatorname{APP}}\left(M_{1}, M_{2}\right)\right\|^{k^{\prime}}$ and $\left\|\underline{\operatorname{APP}}\left(M_{1}, M_{2}\right)\right\|^{k^{\prime}} \neq \square$.

By the assumptions, there exists a $k_{1}^{\prime}$ such that $\left|M_{1}\right|^{k} \sqsubseteq\left\|M_{1}\right\|^{k_{1}}$ and $\left\|M_{1}\right\|^{k_{1}^{\prime}} \neq \square$, and a $k_{2}^{\prime}$ such that $\left|M_{2}\right|^{k} \sqsubseteq\left\|M_{2}\right\|^{k_{2}^{\prime}}$. Take $k^{\prime}=\max \left(k_{1}^{\prime}, k_{2}^{\prime}\right)+1$. Then, noting that for any $M$ and $k^{\prime} \leq k^{\prime \prime},\|M\|^{k^{\prime}} \sqsubseteq\|M\|^{k^{\prime \prime}}$, we get:

$$
\begin{aligned}
& \left|\underline{\operatorname{APP}}\left(M_{1}, M_{2}\right)\right|^{k} \sqsubseteq\left|\underline{\operatorname{APP}}\left(M_{1}, M_{2}\right)\right|^{k+1}=\underline{\operatorname{APP}}\left(\left|M_{1}\right|^{k},\left|M_{2}\right|^{k}\right) \\
& \sqsubseteq \underline{\operatorname{APP}}\left(\left\|M_{1} \mid\right\|_{1}^{\prime},\left\|M_{2}\right\|_{2}^{\prime}\right) \sqsubseteq \underline{\operatorname{APP}}\left(\left\|M_{1}\right\|\left\|^{\max \left(k_{1}^{\prime}, k_{2}^{\prime}\right)},\right\| M_{2} \|^{\max \left(k_{1}^{\prime}, k_{2}^{\prime}\right)}\right) \\
& =\left\|M_{1}\right\|^{\max \left(k_{1}^{\prime}, k_{2}^{\prime}\right) \circledast \lambda M_{1}^{\prime}} \cdot \underline{\operatorname{APP}}\left(M_{1}^{\prime},\left\|M_{2}\right\| \max \left(k_{1}^{\prime}, k_{2}^{\prime}\right)\right) \\
& =\left\|\underline{\operatorname{APP}}\left(M_{1}, M_{2}\right)\right\|^{\max \left(k_{1}^{\prime}, k_{2}^{\prime}\right)+1}=\left\|\underline{\operatorname{APP}}\left(M_{1}, M_{2}\right)\right\|^{k^{\prime}} .
\end{aligned}
$$

For showing completeness, it will also be convenient to disregard the exact variable names occurring in the output tree. Accordingly, we define the (evidently continuous) shape function $\$: \underline{\Lambda} \rightarrow \underline{\Lambda}$, by

$$
\$ \underline{\operatorname{LAM}}\left(x, M_{0}\right)=\underline{\operatorname{LAM}}\left(x_{\$}, \$ M_{0}\right) \underline{\$ \operatorname{VAR}}(x)=\underline{\operatorname{VAR}}\left(x_{\$}\right)=\underline{\operatorname{APP}}\left(M_{1}, M_{2}\right)=\underline{\operatorname{APP}}\left(\$ M_{1}, \$ M_{2}\right)
$$

where $x_{\$}$ is some arbitrary but fixed variable. From the definition of the ordering relation on $\underline{\Lambda}$, it is easy to see that if $M \sqsubseteq M^{\prime}$ but $\$ M^{\prime} \sqsubseteq \$ M$, then $M=M^{\prime}$.

We can now refine the previous characterization of the wrapper functions, to state that they produce representatives that are at least as defined as some given finite tree:

Definition 10 (cf. Definition 4) For any finite $M$, and $l \in \widehat{\Lambda}$, we define the bounded uniform definedness predicate $\operatorname{def}_{M}(l)$ by $\operatorname{def}_{M}(l) \Leftrightarrow \forall n \in \omega . \$ M \sqsubseteq \$(\ln )$. We also write $\operatorname{def}_{M}^{+}(l)$ for $M \neq \square \wedge \operatorname{def}_{M}(l)$. 
Lemma 23 (cf. Lemma 10) The wrapper functions preserve bounded definedness:
a. For all $v \in \mathrm{V}, \operatorname{def}_{\underline{\operatorname{VAR}(x)}}^{+}(\widehat{\operatorname{VAR}}(v))$.
b. If for all $v \in \mathrm{V}$, $\operatorname{def}_{M_{0}}^{+}(f v)$, then $\operatorname{def}_{\underline{\underline{L A M}\left(x, M_{0}\right)}}^{+}(\widehat{\mathrm{LAM}}(f))$.
c. If $\operatorname{def}_{M_{1}}^{+}\left(l_{1}\right)$ and $\operatorname{def}_{M_{2}}\left(l_{2}\right)$, then $\operatorname{def}_{\underline{\mathrm{APP}}\left(M_{1}, M_{2}\right)}^{+}\left(\widehat{\mathrm{APP}}\left(l_{1}, l_{2}\right)\right)$.

Proof: In each case, let an arbitrary $n \in \omega$ be given. Then,

a. We have to show that $\$ \underline{\operatorname{VAR}}(x) \sqsubseteq \$ \underline{\operatorname{VAR}}(v)$. But by the definition of $\$ \cdot$, both sides are simply $\underline{\operatorname{VAR}}\left(x_{\$}\right)$, so the inequality holds.

b. We have to show that $\$ \underline{\operatorname{LAM}}\left(x, M_{0}\right) \sqsubseteq \$\left(f \mathrm{~g}_{n}(n+1) \circledast \lambda M_{0}^{\prime} \cdot \underline{\operatorname{LAM}}\left(\mathrm{g}_{n}, M_{0}^{\prime}\right)\right)$. Let $M_{0}^{\prime}=f \mathrm{~g}_{n}(n+1)$. By the assumption on $f, \operatorname{def}_{M_{0}}^{+}\left(f \mathrm{~g}_{n}\right)$, so $M_{0} \neq \square$ and $\$ M_{0} \sqsubseteq \$ M_{0}^{\prime}$. Hence also $M_{0}^{\prime} \neq \square$, and it only remains to show that $\$ \underline{\operatorname{LAM}}\left(x, M_{0}\right) \sqsubseteq \$ \underline{\mathrm{LAM}}\left(\mathrm{g}_{n}, M_{0}^{\prime}\right)$, which follows immediately from the definition of $\$$.

c. We have to show that $\$ \underline{\operatorname{APP}}\left(M_{1}, M_{2}\right) \sqsubseteq \$\left(l_{1} n \circledast \lambda M_{1}^{\prime} \cdot \underline{\operatorname{APP}}\left(M_{1}^{\prime}, l_{2} n\right)\right)$. Let $M_{1}^{\prime}=l_{1} n$ and $M_{2}^{\prime}=l_{2} n$. By assumptions on $l_{1}$ and $\overline{l_{2}}$, we then get that $M_{1} \neq \square, \$ M_{1} \sqsubseteq \$ M_{1}^{\prime}$, and $\$ M_{2} \sqsubseteq \$ M_{2}^{\prime}$. So, again, we get $M_{1}^{\prime} \neq \square$, and then $\$ \underline{\operatorname{APP}}\left(M_{1}, M_{2}\right) \sqsubseteq \$ \underline{\operatorname{APP}}\left(M_{1}^{\prime}, M_{2}^{\prime}\right)$ by the definition of $\$$.

Lemma 24 (cf. Lemma 11) Let $m \in \Lambda$ and $\rho \in\left[\mathrm{V} \rightarrow D_{\mathrm{r}}\right]$ be such that $\forall x \in$ $F V(m) . \exists l \in \widehat{\Lambda} . \rho(x)=\uparrow l \wedge \operatorname{def}_{\underline{\operatorname{VAR}(x)}}^{+}(l)$. Then, for any $M \in \underline{\Lambda}$ with $M \sqsubseteq\langle m\rangle$,

a. If $M \in \mathcal{B}_{\mathrm{at}}^{\mathrm{fin}}$, then $\exists l . \llbracket m \rrbracket_{\mathrm{r}} \rho=\uparrow l \wedge \operatorname{def}_{M}^{+}(l)$.

b. If $M \in \mathcal{B}_{\mathrm{nf}}^{\text {fin }}$, then $\operatorname{def}_{M}^{+}\left(\downarrow \llbracket m \rrbracket_{\mathrm{r}} \rho\right)$.

c. If $M \in \mathcal{B}_{\mathrm{bt}}^{\mathrm{fin}}$, then $\operatorname{def}_{M}\left(\downarrow \llbracket m \rrbracket_{\mathrm{r}} \rho\right)$.

Proof: By rule induction for $\mathcal{B}^{\text {fin }}$, with respect to the evident combined predicate, giving two cases for each part of the lemma:

a. For Rule (AT-VAR), we have $M=\underline{\operatorname{VAR}}(x)$, so we must have $m=\operatorname{VAR}(x)$. Then $\llbracket m \rrbracket_{\mathrm{r}} \rho=\rho(x)$, and since $x \in F \overline{V(m)}$, the result follows directly from the assumption on $\rho$.

For Rule (AT-APP), we have $M=\underline{\operatorname{APP}}\left(M_{1}, M_{2}\right)$, with $M_{1} \in \mathcal{B}_{\text {at }}^{\text {fin }}$ and $M_{2} \in$ $\mathcal{B}_{\text {bt }}^{\text {fin }}$, so we must have $m=\operatorname{APP}\left(m_{1}, m_{2}\right)$, with $M_{1} \sqsubseteq\left\langle m_{1}\right\rangle$ and $M_{2} \sqsubseteq\left\langle m_{2}\right\rangle$. By $\operatorname{IH}(\mathrm{a})$ on the first premise, there exists an $l_{1}$ such that $\llbracket m_{1} \rrbracket_{\mathrm{r}} \rho=\uparrow l_{1}$ and $\operatorname{def}_{M_{1}}^{+}\left(l_{1}\right)$. Therefore, $\llbracket m \rrbracket_{\mathrm{r}} \rho=\psi_{\mathrm{r}}\left(\operatorname{tm}\left(l_{1}\right)\right)\left(\llbracket m_{2} \rrbracket_{\mathrm{r}} \rho\right)=\uparrow\left(\widehat{\operatorname{APP}}\left(l_{1}, \downarrow\left(\llbracket m_{2} \rrbracket_{\mathrm{r}} \rho\right)\right)\right)$. Take $l_{2}=\downarrow\left(\llbracket m_{2} \rrbracket_{\mathrm{r}} \rho\right)$ and $l=\widehat{\operatorname{APP}}\left(l_{1}, l_{2}\right)$. By $\mathrm{IH}(\mathrm{c})$ on the second premise, $\operatorname{def}_{M_{2}}\left(l_{2}\right)$, so by Lemma $23(\mathrm{c})$, $\operatorname{def}_{M}^{+}(l)$, as required.

b. For Rule (NF-AT), we have $M \in \mathcal{B}_{\text {at }}^{\text {fin }}$. By $\mathrm{IH}(\mathrm{a})$ on the premise, $\llbracket m \rrbracket_{\mathrm{r}} \rho=\uparrow l$, for some $l$ with $\operatorname{def}_{M}^{+}(l)$. But $\downarrow(\uparrow l)=l$, so also $\operatorname{def}_{M}^{+}\left(\downarrow\left(\llbracket m \rrbracket_{\mathrm{r}} \rho\right)\right)$.

For Rule (NF-LAM), we have $M=\underline{\operatorname{LAM}}\left(x, M_{0}\right)$, with $M_{0} \in \mathcal{B}_{\mathrm{nf}}^{\mathrm{fin}}$, so we must have $m=\operatorname{LAM}\left(x, m_{0}\right)$ with $M_{0} \sqsubseteq\left\langle m_{0}\right\rangle$, and thus $\llbracket m \rrbracket_{\mathrm{r}} \rho=\operatorname{fun}\left(\lambda d . \llbracket m_{0} \rrbracket_{\mathrm{r}} \rho[x \mapsto d]\right)$. Expanding the definition of $\downarrow$ for the functional case, we have to show that

$$
\operatorname{def}_{\underline{\underline{L A M}}\left(x, M_{0}\right)}^{+}\left(\widehat{\operatorname{LAM}}\left(\lambda x \cdot \downarrow\left(\llbracket m_{0} \rrbracket_{\mathrm{r}} \rho[x \mapsto \uparrow(\widehat{\mathrm{VAR}}(x))]\right)\right)\right) .
$$


By Lemma 23(b), it suffices to show, for every $v \in \mathrm{V}, \operatorname{def}_{M_{0}}^{+}\left(\downarrow\left(\llbracket m_{0} \rrbracket_{\mathrm{r}} \rho^{\prime}\right)\right)$, where $\rho^{\prime}=\rho[x \mapsto \uparrow(\widehat{\operatorname{VAR}}(v))]$. This follows from $\mathrm{IH}(\mathrm{b})$ on the premise, if for every $x^{\prime} \in F V\left(m_{0}\right)$, there exists an $l$, such that $\rho^{\prime}\left(x^{\prime}\right)=\uparrow l$ and $\operatorname{def}_{\underline{\mathrm{VAR}}\left(x^{\prime}\right)}^{+}(l)$. But for $x^{\prime} \neq x$, we must have $x^{\prime} \in F V(m)$, so this follows from the assumption on $\rho$; and for $x^{\prime}=x$, it follows from Lemma 23(a).

c. For Rule (BT- $\square$ ), we have $M=\square$, so the result follows trivially, since any $m$ satisfies $\operatorname{def}_{\square}\left(\downarrow\left(\llbracket m \rrbracket_{\mathrm{r}} \rho\right)\right)$.

For Rule (BT-NF), we have $M \in \mathcal{B}_{\mathrm{nf}}^{\text {fin }}$, so by $\mathrm{IH}(\mathrm{b})$ on the premise, we get $\operatorname{def}_{M}^{+}\left(\downarrow\left(\llbracket m \rrbracket_{\mathrm{r}} \rho\right)\right)$, from which also $\operatorname{def}_{M}\left(\downarrow\left(\llbracket m \rrbracket_{\mathrm{r}} \rho\right)\right)$.

We can now show the main completeness lemma:

Lemma 25 Let $m \in \Lambda^{\Delta}$. If $M \leftrightarrow^{\dagger} m$ and $M \in \mathcal{B}_{\mathrm{bt}}^{\inf }$ then $\$ M \sqsubseteq \$ \mathrm{bt}_{\Delta}(m)$.

Proof: Since for any $\lambda$-tree $M, M=\bigsqcup_{k}|M|^{k}$ (Lemma 16), by continuity of $\$$, we get the desired result from $\bigsqcup_{k} \$|M|^{k} \sqsubseteq \$ \mathrm{bt}_{\Delta}(m)$. By the definition of $\sqcup$, it thus suffices to show, for all $k$, that $\$|M|^{k} \sqsubseteq \$ \mathrm{bt}_{\Delta}(m)$.

Let $k$ be given. By Lemma $22(\mathrm{c})$, there exists a $k^{\prime}$ such that $|M|^{k} \sqsubseteq\|M\|^{k^{\prime}}$. From the definition of $M \leftrightarrow^{\dagger} m$, we get that, for this $k^{\prime}$, there exists an $m^{\prime} \in \Lambda$, such that $|M|^{k^{\prime}} \sqsubseteq\left\langle m^{\prime}\right\rangle$ and $m \leftrightarrow m^{\prime}$. Since $\|M\|^{k^{\prime}} \sqsubseteq|M|^{k^{\prime}}$ (Lemma 22(b)), we must also have $\|M\|^{k^{\prime}} \sqsubseteq\left\langle m^{\prime}\right\rangle$.

Let $\rho_{0}=\lambda x \cdot \uparrow(\widehat{\operatorname{VAR}}(x))$; by Lemma $23($ a), this clearly satisfies the condition on $\rho$ in Lemma 24. Since $\|M\|^{k^{\prime}} \in \mathcal{B}_{\mathrm{bt}}^{\text {fin }}$ (Lemma 22(a)), Lemma 24(c) gives us that $\operatorname{def}_{\|M\| k^{\prime}}\left(\downarrow\left(\llbracket m^{\prime} \rrbracket_{\mathrm{r}} \rho_{0}\right)\right)$, so in particular $\$\|M\|^{k^{\prime}} \sqsubseteq \$\left(\downarrow\left(\llbracket m^{\prime} \rrbracket_{\mathrm{r}} \rho_{0}\right) \sharp \Delta\right)$. Thus, using model soundness, $\$\|M\|^{k^{\prime}} \sqsubseteq \$\left(\downarrow\left(\llbracket m \rrbracket_{\mathrm{r}} \rho_{0}\right) \sharp \Delta\right)=\$ \mathrm{bt}_{\Delta}(m)$. Finally, from $|M|^{k} \sqsubseteq$ $\|M\|^{k^{\prime}}$, we get $\$|M|^{k} \sqsubseteq \$\|M\|^{k^{\prime}}$, and thus $\$|M|^{k} \sqsubseteq \$ \mathrm{bt}_{\Delta}(m)$, as required.

Theorem 5 (cf. Theorem 2) bt ${ }_{\Delta}$ from Definition 7 is a Böhm-tree normalization function on $\Lambda^{\Delta}$, i.e., for all $m, m^{\prime} \in \Lambda^{\Delta}$,

a. (soundness) $\mathrm{bt}_{\Delta}(m) \leftrightarrow{ }_{\Delta}^{\dagger} m$ and $\mathrm{bt}_{\Delta}(m) \in \mathcal{B}_{\mathrm{bt}}^{\text {inf }}$.

b. (identification) If $m \leftrightarrow m^{\prime}$, then $\mathrm{bt}_{\Delta}(m)=\mathrm{bt}_{\Delta}\left(m^{\prime}\right)$.

c. (completeness) bt $\Delta(m)$ is maximal among $M \in \mathcal{B}_{\mathrm{bt}}^{\inf }$ such that $M \leftrightarrow^{\dagger} m$.

Proof: (Soundness) and (identification) are shown verbatim as in Theorem 2 (using Lemma 21(a) instead of Lemma 5(a)), with the unsurprising exception that unfolding the new definition for $\lesssim$ (using Definition 8 instead of Definition 3), again taking $n=$ $\sharp \Delta$, yields bt $\Delta(m)=\square \vee \mathrm{bt}_{\Delta}(m) \in \mathcal{B}_{\mathrm{nf}}^{\inf }$, from which we get the desired bt $\Delta(m) \in \mathcal{B}_{\mathrm{bt}}^{\inf }$ by Rule BT- $\square$ or Rule BT-NF, respectively.

(Completeness) Let $M \in \mathcal{B}_{\mathrm{bt}}^{\text {inf }}$ with $M \leftrightarrow^{\dagger} m$ be given; we must show that $M$ cannot be strictly greater than bt $\Delta(m)$. So assume that bt $\Delta(m) \sqsubseteq M$. By Lemma 25 , $\$ M \sqsubseteq \$ \mathrm{bt}_{\Delta}(m)$, so we must actually have $\mathrm{bt}_{\Delta}(m)=M$.

From downwards closure of $\leftrightarrow^{\dagger}$, we get a simple, intuitive characterization of soundness: in any finite approximation (not necessarily a level-uniform cut) of bt $\Delta(m)$, we can replace all holes $\square$ with proper terms, to obtain a term convertible to the original $m$. (In particular, if $\mathrm{bt}_{\Delta}(m) \neq \square$, by the inversion principle for $\mathcal{B}_{\mathrm{bt}}^{\text {inf }}$, we see 


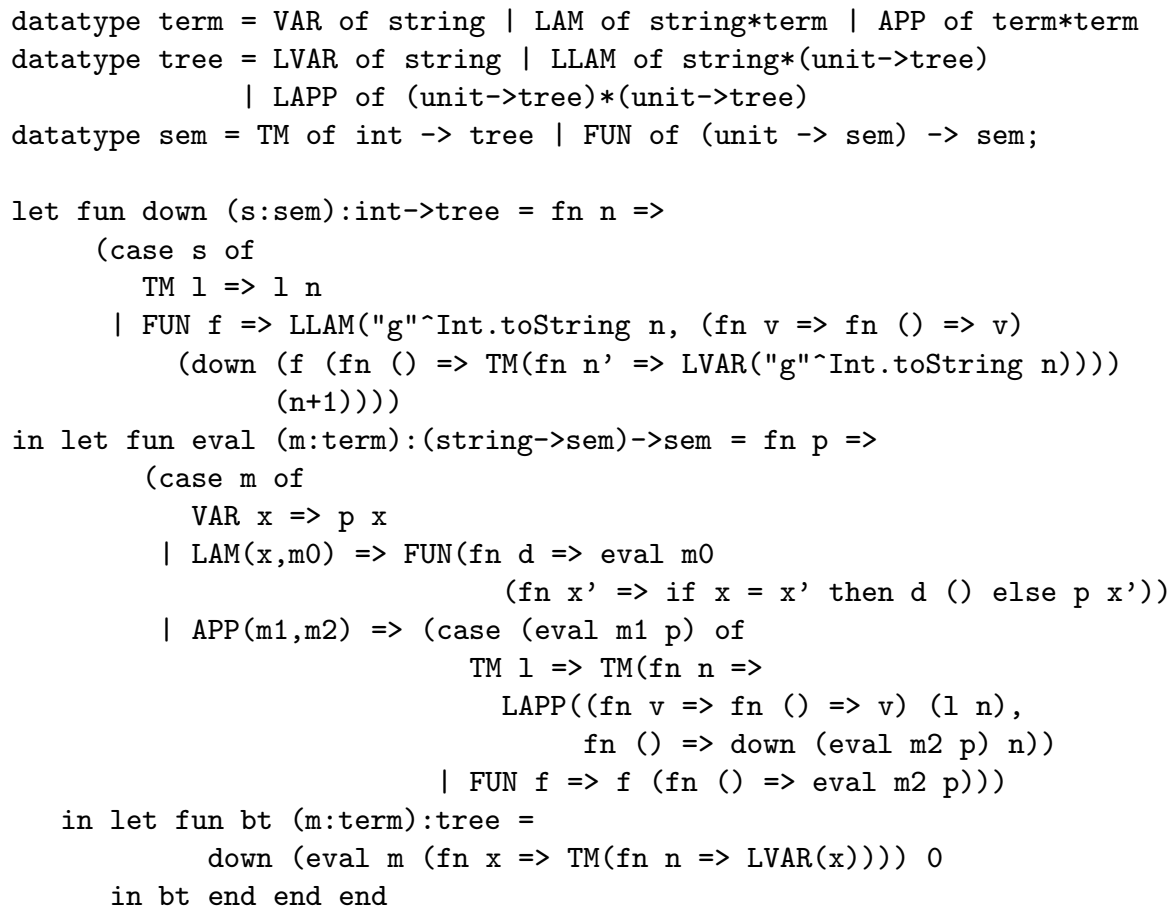

Figure 4: The Böhm normalization algorithm, BT .

that the original term must have at least a head normal form.) On the other hand, completeness says that no such replacement for a hole already present in $\mathrm{bt}_{\Delta}(m)$ can have even a head normal form, since this would contradict that the result tree was maximal.

Like in the finitary case, the characterization of normal forms for soundness and completeness is based on $\beta$-normalization only. If we restricted our definition of Böhm trees to only $\alpha$-normal ones (i.e., using a fixed naming convention for bound variables), instead of saying that the output was a maximal Böhm tree compatible with the input, we would have that it was the largest.

\subsection{An implementation of the construction}

As before, the development of an actual algorithm and its proof of correctness is straightforward, given the domain-theoretic construction. Unsurprisingly, we shall need to identify $\square$ with divergence, to obtain a computable algorithm (shown in Figure 4), returning so-called effective Böhm trees.

As before, we assume $\llbracket$ term $\rrbracket^{\mathrm{ml}}=\Lambda$, and take $T=\llbracket$ tree $\rrbracket^{\mathrm{ml}}$ and $S=\llbracket$ sem $\rrbracket^{\mathrm{ml}}$. Note that tree is overly lazy when representing Böhm trees and we therefore need to strictify the representations of $\widehat{\mathrm{LAM}}$ and $\widehat{\mathrm{APP}}$ explicitly, using the idiom ( $\mathrm{f} \mathrm{v}=>$ $\mathrm{fn}() \Rightarrow \mathrm{v})$. As remarked in Section 5.2, the latter strictification is in fact optional, but advantageous from an efficiency perspective. 
Lemma 26 There exists an isomorphism $i_{\underline{\Lambda} T}: \underline{\Lambda} \cong T_{\perp}$, satisfying:
a. $i_{\underline{\Lambda} T}(\square)=\perp_{T_{\perp}}$.
b. For all $x, i_{\underline{\Lambda} T}(\underline{\operatorname{VAR}}(x))=\left\lfloor\iota_{\operatorname{LVAR}}(x)\right\rfloor$.
c. For all $x$ and $M_{0}, i_{\underline{\Lambda} T}\left(\underline{\operatorname{LAM}}\left(x, M_{0}\right)\right)=\left\lfloor\iota_{\mathrm{LLAM}}\left(x, \lambda u^{1} \cdot i_{\underline{\Lambda} T}\left(M_{0}\right)\right)\right\rfloor$.
d. For all $M_{1}$ and $M_{2}, i_{\underline{\Lambda} T}\left(\underline{\operatorname{APP}}\left(M_{1}, M_{2}\right)\right)=\left\lfloor\iota_{\mathrm{LAPP}}\left(\lambda u^{1} . i_{\underline{\Lambda} T}\left(M_{1}\right), \lambda u^{1} . i_{\underline{\Lambda} T}\left(M_{2}\right)\right)\right\rfloor$.

Proof: See Appendix B.2

We can also relate the our two variants of strict extension:

Lemma 27 For $M \in \underline{\Lambda}$ and $f: \underline{\Lambda} \rightarrow \underline{\Lambda}, i_{\underline{\Lambda} T}(M \circledast f)=i_{\underline{\Lambda} T}(M) \star \lambda t^{T} . i_{\underline{\Lambda} T}\left(f\left(i_{\underline{\Lambda} T}^{-1}(\lfloor t\rfloor)\right)\right)$.

Proof: By cases on $M$ : if $M=\square$, then $i_{\underline{\Lambda} T}(M)=\perp$, and hence

$$
\begin{aligned}
& i_{\underline{\Lambda} T}(M \circledast f)=i_{\underline{\Lambda} T}(\square)=\perp=\perp \star \lambda t . i_{\underline{\Lambda} T}\left(f\left(i_{\underline{\Lambda} T}^{-1}(\lfloor t\rfloor)\right)\right) \\
& =i_{\underline{\Lambda} T}(M) \star \lambda t . i_{\underline{\Lambda} T}\left(f\left(i_{\underline{\Lambda} T}^{-1}(\lfloor t\rfloor)\right)\right) .
\end{aligned}
$$

On the other hand, if $M \neq \square$, then $i_{\underline{\Lambda} T}(M)=\lfloor t\rfloor$ for some $t \in T$, and thus

$$
\begin{aligned}
& i_{\underline{\Lambda} T}(M \circledast f)=i_{\underline{\Lambda} T}(f M)=i_{\underline{\Lambda} T}\left(f\left(i_{\underline{\Lambda}}^{-1}\left(i_{\underline{\Lambda} T}(M)\right)\right)\right)=i_{\underline{\Lambda} T}\left(f\left(i_{-1}^{-1}(\lfloor t\rfloor)\right)\right) \\
& =\lfloor t\rfloor \star \lambda t . i_{\underline{\Lambda} T}\left(f\left(i_{\underline{\Lambda} T}^{-1}(\lfloor t\rfloor)\right)\right)=i_{\underline{\Lambda} T}(M) \star \lambda t . i_{\underline{\Lambda} T}\left(f\left(i_{\underline{\Lambda} T}^{-1}(\lfloor t\rfloor)\right)\right) .
\end{aligned}
$$

Like in the finitary case, we have chosen $\mathrm{N}=\mathbb{Z}$, so that $\widehat{\Lambda}=[\mathbb{Z} \rightarrow \underline{\Lambda}]$ is the base domain in the definition of $D_{\mathrm{r}}$, whereas in $S$, we use the isomorphic $\left[\mathbb{Z} \rightarrow T_{\perp}\right]$. Therefore, we get a slightly more complicated characterization of the relationship between the two residualizing models:

Lemma 28 (cf. Lemma 12) There exists an isomorphism $i_{D S}: D_{\mathrm{r}} \cong S_{\perp}$, satisfying:

a. For all $l \in \widehat{\Lambda}, i_{D S}(\operatorname{tm}(l))=\left\lfloor\iota_{\mathrm{TM}}\left(\lambda n^{\mathbb{Z}} \cdot i_{\underline{\Lambda} T}(l n)\right)\right\rfloor$.

b. For all $f \in\left[D_{\mathrm{r}} \rightarrow D_{\mathrm{r}}\right], i_{D S}(\operatorname{fun}(f))=\left\lfloor\iota_{\mathrm{FUN}}\left(\lambda t^{1 \rightarrow S_{\perp}} . i_{D S}\left(f\left(i_{D S}^{-1}(t *)\right)\right)\right)\right\rfloor$.

c. $i_{D S}\left(\perp_{D_{\mathrm{r}}}\right)=\perp_{S_{\perp}}$

Proof: See Appendix B.1.

We are now in position to relate the central domain-theoretic functions to the denotations of their syntactic counterparts:

Lemma 29 (cf. Lemma 13) For all $d \in D_{\mathrm{r}}$ and $n \in \mathbb{Z}$,

$$
i_{\underline{\Lambda} T}(\downarrow d n)=i_{D S}(d) \star \lambda s^{S} \cdot \theta_{\text {down }} s \star \lambda l^{\left[\mathbb{Z} \rightarrow T_{\perp}\right]} . \ln
$$

Proof: By fixed-point induction on $\Phi \times \Theta_{\text {down }}$ (where $\Phi$ is as in the proof of Lemma 8 , but with the Böhm-tree definitions), using the predicate $R \subseteq\left[D_{\mathrm{r}} \rightarrow \widehat{\Lambda}\right] \times\left[S \rightarrow \widehat{\Lambda}_{\perp}\right]$ defined by

$$
R=\left\{(\varphi, \theta) \mid \forall d \in D_{\mathrm{r}}, n \in \mathbb{Z} . i_{\underline{\Lambda} T}(\varphi d n)=i_{D S}(d) \star \lambda s^{S} . \theta s \star \lambda l^{\left[\mathbb{Z} \rightarrow T_{\perp}\right]} . l n\right\}
$$

The proof proceeds similarly to the proof of Lemma 13, expect that the isomorphism $i_{\underline{\Lambda} T}$ must now also be accounted for. Assume that $(\varphi, \theta) \in R$, and let arbitrary $d$ and $n$ be given. The cases $d=\perp_{D_{\mathrm{r}}}$ and $d=\operatorname{tm}(l)$ are essentially unchanged, but now using Lemmas 26(a) and 28(a), respectively. 
Case $d=$ fun $(f)$ : Let $\xi=\emptyset\left[\right.$ down $\left.\mapsto \theta, \mathbf{s} \mapsto \iota_{\mathrm{FUN}}\left(\lambda t . i_{D S}\left(f\left(i_{D S}^{-1}(t *)\right)\right)\right)\right]$ and let $\xi^{\prime}=$ $\xi\left[\mathrm{n} \mapsto n, \mathrm{f} \mapsto\left(\lambda t . i_{D S}\left(f\left(i_{D S}^{-1}(t *)\right)\right)\right)\right]$. Then,

$$
\begin{aligned}
& i_{D S}(d) \star \lambda s^{S} . \Theta_{\text {down }}(\theta) s \star \lambda l l^{\left[\mathbb{Z} \rightarrow T_{\perp}\right]} . l n \\
& =i_{D S}(\operatorname{fun}(f)) \star \lambda s^{S} \cdot \Theta_{\text {down }}(\theta) s \star \lambda l\left[l^{\left[\mathbb{Z} \rightarrow T_{\perp}\right]} . l n\right. \\
& =\left\lfloor\iota_{\mathrm{FUN}}\left(\lambda t . i_{D S}\left(f\left(i_{D S}^{-1}(t *)\right)\right)\right\rfloor \star \lambda s^{S} . \Theta_{\text {down }}(\theta) s \star \lambda l^{\left[\mathbb{Z} \rightarrow T_{\perp}\right]} . l n\right.
\end{aligned}
$$

(by Lemma 28(b))

$=\llbracket \mathrm{fn} \mathrm{n} \Rightarrow($ case $\mathrm{s}$ of $\ldots \mid$ FUN $\mathrm{f} \Rightarrow$ LLAM $\ldots) \rrbracket^{\mathrm{ml}} \xi \star \lambda l^{\left[\mathbb{Z} \rightarrow T_{\perp}\right]} . l n$

$=\llbracket \operatorname{LLAM}\left(" \mathrm{~g} "\right.$ "Int.toString $(\mathrm{n}),(\mathrm{fn} \mathrm{v} \Rightarrow \mathrm{fn}() \Rightarrow \mathrm{v})(\ldots) \rrbracket^{\mathrm{ml}} \xi^{\prime}$

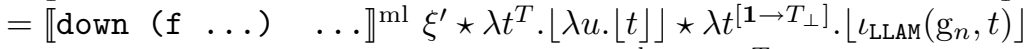

$=\llbracket$ down $(f(f \mathrm{fn}() \Rightarrow \ldots))(\mathrm{n}+1) \rrbracket^{\mathrm{ml}} \xi^{\prime} \star \lambda t^{T} \cdot\left\lfloor\iota_{\mathrm{LLAM}}\left(\mathrm{g}_{n}, \lambda u \cdot\lfloor t\rfloor\right)\right\rfloor$

$=\underbrace{\llbracket f(\ldots) \rrbracket^{\mathrm{ml}} \xi^{\prime}}_{s^{\prime}} \star \lambda s^{S} \cdot \theta s \star \lambda l l^{\left[\mathbb{Z} \rightarrow T_{\perp}\right]} \cdot l(n+1) \star \lambda t^{T} \cdot\left\lfloor\iota_{\mathrm{LLAM}}\left(g_{n}, \lambda u \cdot\lfloor t\rfloor\right)\right\rfloor$

Now,

$s^{\prime}$

$=\llbracket \mathrm{f}\left(\mathrm{fn}() \Rightarrow \operatorname{TM}\left(\mathrm{fn} \mathrm{n} \mathrm{n}^{\prime} \Rightarrow \operatorname{LVAR}(" \mathrm{~g} "\right.\right.$ "Int.toString $\left.\left.(\mathrm{n}))\right)\right) \rrbracket^{\mathrm{ml}} \xi^{\prime}$

$=\left\lfloor\left(\lambda t \cdot i_{D S}\left(f\left(i_{D S}^{-1}(t *)\right)\right)\right)\right\rfloor \star \lambda g \cdot\left\lfloor\lambda u \cdot \llbracket \operatorname{TM}(\mathrm{fn} \ldots.) \rrbracket^{\mathrm{ml}} \xi^{\prime}\right\rfloor \star \lambda a \cdot g a$

$=i_{D S}\left(f\left(i_{D S}^{-1}\left(\left\lfloor\iota_{\mathrm{TM}}\left(\lambda n^{\prime \mathbb{Z}} \cdot\left\lfloor\iota_{\mathrm{LVAR}}\left(\mathrm{g}_{n}\right)\right\rfloor\right)\right\rfloor\right)\right)\right)$

$=i_{D S}\left(f\left(i_{D S}^{-1}\left(\left\lfloor\iota_{\mathrm{TM}}\left(\lambda n^{\prime \mathbb{Z}} \cdot i_{\underline{\Lambda} T}\left(\underline{\operatorname{VAR}}\left(\mathrm{g}_{n}\right)\right)\right)\right\rfloor\right)\right)\right)$

$=i_{D S}\left(f\left(\operatorname{tm}\left(\lambda n^{\prime \mathbb{Z}} \cdot \underline{\operatorname{VAR}}\left(\overline{\mathrm{g}}_{n}\right)\right)\right)\right)$

$=i_{D S}\left(f\left(\uparrow\left(\widehat{\operatorname{VAR}}\left(\mathrm{g}_{n}\right)\right)\right)\right)$

(by Lemma 26(b))

(by Lemma 28(a))

(by Def. of $\widehat{\mathrm{VAR}}$ and $\uparrow$ )

The fixed point assumption on $\varphi$ and $\theta$ says that for all $d^{\prime} \in D_{\mathrm{r}}$ and $n^{\prime} \in \mathbb{Z}$, $i_{\underline{\Lambda} T}\left(\varphi d^{\prime} n^{\prime}\right)=i_{D S}\left(d^{\prime}\right) \star \lambda s^{S} . \theta s \star \lambda l^{\left[\mathbb{Z} \rightarrow T_{\perp}\right]} . l n^{\prime}$. Taking $d^{\prime}=f\left(\uparrow\left(\widehat{\operatorname{VAR}}\left(\mathrm{g}_{n}\right)\right)\right)$ and $\overline{n^{\prime}}=n+1$, we continue:

$$
\begin{aligned}
& \llbracket f(\ldots) \rrbracket^{\mathrm{ml}} \xi^{\prime} \star \lambda s^{S} \cdot \theta s \star \lambda l^{\left[\mathbb{Z} \rightarrow T_{\perp}\right]} . l(n+1) \star \lambda t^{T} \cdot\left\lfloor\iota_{\mathrm{LLAM}}\left(\mathrm{g}_{n}, \lambda u .\lfloor t\rfloor\right)\right\rfloor \\
& =i_{\underline{\Lambda} T}\left(\varphi\left(f\left(\uparrow\left(\widehat{\operatorname{VAR}}\left(\mathrm{g}_{n}\right)\right)\right)\right)(n+1)\right) \star \lambda t^{T} \cdot\left\lfloor\iota_{\mathrm{LLAM}}\left(\mathrm{g}_{n}, \lambda u \cdot\lfloor t\rfloor\right)\right\rfloor
\end{aligned}
$$

Similarly,

$$
\begin{aligned}
& i_{\underline{\Lambda} T}(\Phi(\varphi) d n) \\
& =i_{\underline{\Lambda} T}(\Phi(\varphi)(\operatorname{fun}(f)) n) \\
& =i_{\underline{\Lambda} T}\left(\widehat{\mathrm{LAM}}\left(\lambda x^{\mathrm{V}} \cdot \varphi(f(\uparrow(\widehat{\mathrm{VAR}}(x))))\right) n\right) \\
& \left.=i_{\underline{\Lambda} T}\left(\varphi\left(f\left(\uparrow\left(\widehat{\mathrm{VAR}}\left(\mathrm{g}_{n}\right)\right)\right)\right)(n+1) \circledast \lambda M \underline{\Lambda} \cdot \underline{\operatorname{LAM}}\left(\mathrm{g}_{n}, M\right)\right) \quad \text { (by Def. of } \widehat{\mathrm{LAM}}\right) \\
& =i_{\underline{\Lambda} T}\left(\varphi\left(f\left(\uparrow\left(\widehat{\operatorname{VAR}}\left(\mathrm{g}_{n}\right)\right)\right)\right)(n+1)\right) \star \lambda t^{\prime T} . i_{\underline{\Lambda} T}\left(\underline{\operatorname{LAM}}\left(\mathrm{g}_{n}, i_{\underline{\Lambda T}}^{-1}(\lfloor t\rfloor)\right)\right) \\
& =i_{\underline{\Lambda} T}\left(\varphi\left(f\left(\uparrow\left(\widehat{\operatorname{VAR}}\left(\mathrm{g}_{n}\right)\right)\right)\right)(n+1)\right) \star \lambda t^{\prime T} \cdot\left\lfloor\iota_{\text {LLAM }}\left(\mathrm{g}_{n}, \lambda u \cdot\lfloor t\rfloor\right)\right\rfloor
\end{aligned}
$$

(by Lemma 26(c))

Lemma 30 (cf. Lemma 14) For all $m \in \Lambda, \rho \in\left[\mathrm{V} \rightarrow D_{\mathrm{r}}\right]$, and $\varrho \in\left[\mathrm{V} \rightarrow S_{\perp}\right]$, such that $\forall x \in F V(m) . i_{D S}(\rho(x))=\varrho(x), i_{D S}\left(\llbracket m \rrbracket_{\mathrm{r}} \rho\right)=\theta_{\text {eval }} m \star \lambda g . g \varrho$.

Proof: By structural induction on $m$. The proof of Lemma 14 can be reused verbatim (using Lemma 28 instead of Lemma 12), except for the single subcase of application where the eval definitions actually differ.

We now establish this subcase, which is to be understood in the corresponding context from the proof of Lemma 14. 
Case $m=\operatorname{APP}\left(m_{1}, m_{2}\right)$ :

Case $\llbracket m_{1} \rrbracket_{\mathrm{r}} \rho=\operatorname{tm}\left(l_{1}\right)$ : By Lemma $28(\mathrm{a})$,

$$
\begin{aligned}
& \theta_{\text {eval }} m \star \lambda g . g \varrho \\
& =\llbracket \operatorname{case}(\text { eval } \mathrm{m} 1 \mathrm{p}) \text { of } \mathrm{TM} I \Rightarrow \operatorname{TM}(\mathrm{fn} \mathrm{n} \ldots) \mid \ldots \rrbracket^{\mathrm{ml}} \xi^{\prime \prime} \\
& =\llbracket \operatorname{TM}(\operatorname{fn} \mathrm{n}=>\operatorname{LAPP}(\ldots)) \rrbracket^{\mathrm{ml}} \xi^{\prime \prime}\left[1 \mapsto \lambda n \cdot i_{\underline{\Lambda} T}\left(l_{1} n\right)\right] \\
& =\left\lfloor\iota_{\mathrm{TM}}(\underbrace{\lambda n^{\mathbb{Z}} \cdot \llbracket \operatorname{LAPP}(\ldots) \rrbracket^{\mathrm{ml}} \xi^{\prime \prime}\left[1 \mapsto \lambda n . i_{\underline{\Lambda} T}\left(l_{1} n\right), \mathrm{n} \mapsto n\right]}_{l})\right\rfloor
\end{aligned}
$$

Similarly,

$$
\begin{aligned}
& i_{D S}\left(\llbracket m \rrbracket_{\mathrm{r}} \rho\right) \\
& =i_{D S}\left(\llbracket \operatorname{APP}\left(m_{1}, m_{2}\right) \rrbracket_{\mathrm{r}} \rho\right) \\
& =i_{D S}\left(\psi_{\mathrm{r}}\left(\operatorname{tm}\left(l_{1}\right)\right)\left(\llbracket m_{2} \rrbracket_{\mathrm{r}} \rho\right)\right) \\
& =i_{D S}\left(\uparrow \widehat{\operatorname{APP}}\left(l_{1}, \downarrow\left(\llbracket m_{2} \rrbracket_{\mathrm{r}} \rho\right)\right)\right) \\
& =i_{D S}\left(\operatorname{tm}\left(\widehat{\operatorname{APP}}\left(l_{1}, \downarrow\left(\llbracket m_{2} \rrbracket_{\mathrm{r}} \rho\right)\right)\right)\right) \\
& =\left\lfloor\iota_{\mathrm{TM}}\left(\lambda n \cdot i_{\underline{\Lambda} T}\left(\widehat{\operatorname{APP}}\left(l_{1}, \downarrow\left(\llbracket m_{2} \rrbracket_{\mathrm{r}} \rho\right)\right) n\right)\right)\right\rfloor \\
& =\left\lfloor\iota_{\mathrm{TM}}(\underbrace{\lambda n \cdot i_{\underline{\Lambda} T}\left(l_{1} n \circledast \lambda M_{1}^{\prime} \cdot \underline{\operatorname{APP}}\left(M_{1}^{\prime}, \downarrow\left(\llbracket m_{2} \rrbracket_{\mathrm{r}} \rho\right) n\right)\right)}_{l^{\prime}})\right\rfloor
\end{aligned}
$$

Again, it remains to show that $l=l^{\prime}$. Let any $n \in \mathbb{Z}$ be given.

Let $\xi^{\prime \prime \prime}=\xi^{\prime \prime}\left[1 \mapsto \lambda n . i_{\underline{\Lambda} T}\left(l_{1} n\right), \mathrm{n} \mapsto n\right]$. We calculate:

$$
\begin{aligned}
& l n \\
& =\llbracket \operatorname{LAPP}((\mathrm{fn} \mathrm{v} \Rightarrow \mathrm{fn}() \Rightarrow \mathrm{v})(\mathrm{l} \mathrm{n}), \mathrm{fn}() \Rightarrow \ldots) \rrbracket^{\mathrm{ml}} \xi^{\prime \prime \prime} \\
& =i_{\underline{\Lambda} T}\left(l_{1} n\right) \star \lambda t_{1} \cdot \llbracket \text { fn } \quad() \Rightarrow \ldots \rrbracket^{\mathrm{ml}} \xi^{\prime \prime \prime} \star \lambda t_{2} \cdot\left\lfloor\iota_{\mathrm{LAPP}}\left(\lambda u \cdot\left\lfloor t_{1}\right\rfloor, t_{2}\right)\right\rfloor \\
& =i_{\underline{\Lambda} T}\left(l_{1} n\right) \star \lambda t_{1} \cdot\left\lfloor\iota_{\text {LAPP }}\left(\lambda u \cdot\left\lfloor t_{1}\right\rfloor, \lambda u \cdot \llbracket \text { down (eval m2 p) } \mathrm{n} \rrbracket^{\mathrm{ml}} \xi^{\prime \prime \prime}\right)\right\rfloor \\
& =i_{\underline{\Lambda} T}\left(l_{1} n\right) \star \lambda t_{1} \cdot\left\lfloor\iota_{\text {LAPP }}\left(\lambda u .\left\lfloor t_{1}\right\rfloor, \lambda u . i_{D S}\left(\llbracket m_{2} \rrbracket_{\mathrm{r}} \rho\right) \star \lambda s . \theta_{\text {down }} s \star \lambda l_{2} . l_{2} n\right)\right\rfloor \\
& \left.=i_{\underline{\Lambda} T}\left(l_{1} n\right) \star \lambda t_{1} \cdot\left\lfloor\iota_{\mathrm{LAPP}}\left(\lambda u .\left\lfloor t_{1}\right\rfloor, \lambda u . i_{\underline{\Lambda} T}\left(\downarrow\left(\llbracket m_{2} \rrbracket_{\mathrm{r}} \rho\right) n\right)\right)\right\rfloor \quad \text { (by Lemma } 29\right) \\
& \left.=i_{\underline{\Lambda} T}\left(l_{1} n\right) \star \lambda t_{1} \cdot i_{\underline{\Lambda} T}\left(\underline{\mathrm{APP}}\left(i_{\underline{\Lambda}}^{-1}\left(\left\lfloor t_{1}\right\rfloor\right), \downarrow\left(\llbracket m_{2} \rrbracket_{\mathrm{r}} \rho\right) n\right)\right) \quad \text { (by Lemma } 26(\mathrm{~d})\right) \\
& =i_{\underline{\Lambda} T}\left(l_{1} n \circledast \lambda M_{1} \cdot \underline{\operatorname{APP}}\left(M_{1}, \downarrow \bar{\downarrow}\left(\llbracket m_{2} \rrbracket_{\mathrm{r}} \rho\right) n\right)\right) \\
& =l^{\prime} n
\end{aligned}
$$

Since $n$ was arbitrary, $l=l^{\prime}$.

Theorem 6 (cf. Lemma 15) For all $m \in \Lambda, i_{\underline{\Lambda} T}(\mathrm{bt}(m))=\theta_{\mathrm{bt}} m$.

Proof: Let $m$ be given, and let $\xi=\emptyset\left[\right.$ down $\mapsto \theta_{\text {down }}$, eval $\mapsto \theta_{\text {eval }}$, bt $\left.\mapsto \theta_{\text {bt }}, \mathrm{m} \mapsto m\right]$. Let further $\left\lfloor\varrho=\llbracket\right.$ fn $\mathrm{x} \Rightarrow \operatorname{TM}($ fn $\mathrm{n} \Rightarrow \operatorname{LVAR}(\mathrm{x})) \rrbracket^{\mathrm{ml}} \xi$ and $\rho=\left(\lambda x^{\mathrm{V}} \cdot \uparrow(\widehat{\operatorname{VAR}}(x))\right)$.

We first verify that $\varrho$ and $\rho$ satisfy the requirements of Lemma 30, namely that for all $x^{\prime} \in \mathrm{V} \supset F V(m)$, 


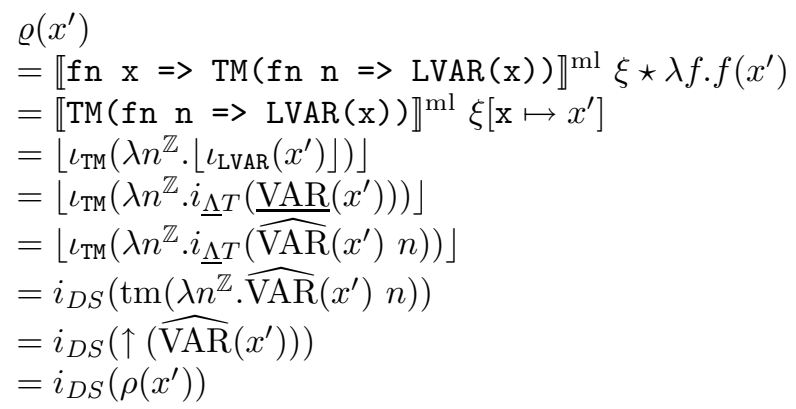

Hence, by a single unrolling of the fixed-point equation $\theta_{\mathrm{bt}}=\Theta_{\mathrm{bt}}\left(\theta_{\mathrm{bt}}\right)$,

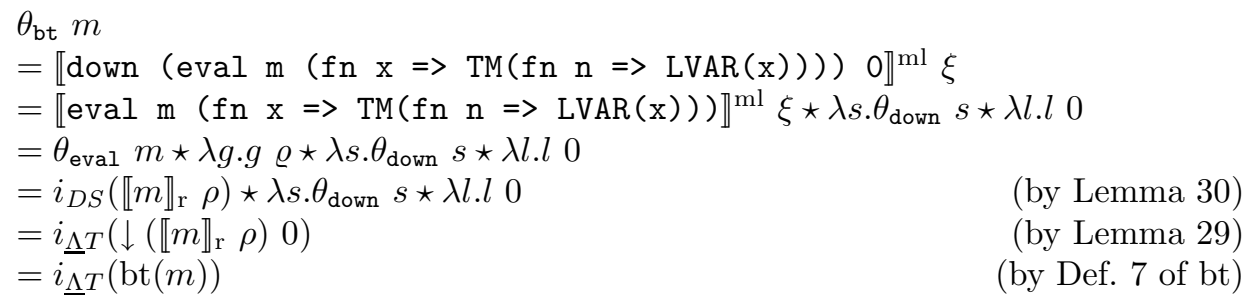

We thus have that the concrete Böhm-tree algorithm is denotationally correct (up to isomorphism). However, BT, although well-typed and closed, is not a complete program, since tree is not a ground type. Hence, unlike for $N O R M$, we cannot readily observe the program output: we first need a formal model of observation of Böhm trees.

\subsection{Observing Böhm trees}

\subsubsection{Computations with infinite results}

When the output of the normalizer is a partial, infinitary data structure, it is far less clear what to consider a legitimate notion of observation of the output. In particular, unlike linear streams, which can be naturally produced and printed incrementally, general trees need either a concept of "fair" autonomous production (every non- $\square$ node will eventually be printed), or a model based on interaction, where an independent observer explicitly asks for successive nodes of the tree, while avoiding branches that are (or might be) $\square$. Properly formalizing each of these in the context of our simple functional language, would be far beyond the scope of the present paper, however.

Instead, we will consider a very simple model of observation, where the observer can only ask about one specific node in the tree, for each run; the notion of interaction is thus lifted out as an extralinguistic concept into multiple (possibly even concurrent) top-level evaluations. (Of course, since the language fragment we consider is pure, many subcomputations can be shared across such evaluations; but our denotational model deliberately does not account for such quantitative aspects.) This approach will still allow us to state precisely that we can correctly inspect any reachable part of the output tree, and observationally distinguish any non-identical trees.

To keep the construction concise in our limited ML fragment, we use a uniform, numeric indexing scheme for nodes. In general, for any finitely branching (but potentially infinitely deep) rooted tree, we can associate a unique natural number to each 
node as follows: the root node has index 0 , and for a node with index $n$ in the $i$ 'th subtree of a $k$-ary root node, its index in the whole tree is $n \cdot k+i$. That is, if we consider a tree to be a node label $a$, and $k$ (possibly 0) subtrees, we access the label of the $n$ 'th node of a tree $t, t @ n$, as follows:

$$
a\left(t_{1}, \ldots, t_{k}\right) @ 0=a(0 \leq k) \quad a\left(t_{1}, \ldots, t_{k}\right) @ n \cdot k+i=t_{i} @ n_{(1 \leq i \leq k)}
$$

Note that the only invalid indices are those that would correspond to subtrees of a zero-ary (i.e., leaf) node.

\subsubsection{Observing $\lambda$-trees}

For the specific case of $\lambda$-trees, we must also take into account partiality, and the fact that various nodes have different information as the label. Accordingly, we define the set of observation results by

$$
\mathrm{O}=\{\operatorname{VR}(v) \mid v \in \mathrm{V}\} \cup\{\mathrm{LM}(v) \mid v \in \mathrm{V}\} \cup\{\mathrm{AP}\} \cup\{\mathrm{ER}\}
$$

and define the operation $\cdot @ \cdot: \underline{\Lambda} \times \omega \rightarrow \mathrm{O}_{\perp}$ by course-of-values induction on the second argument:

$$
\begin{gathered}
\square @ n=\perp \quad \underline{\operatorname{VAR}}(v) @ 0=\lfloor\operatorname{VR}(v)\rfloor \quad \underline{\operatorname{VAR}}(v) @ n+1=\lfloor\mathrm{ER}\rfloor \\
\underline{\operatorname{LAM}}\left(v, M_{0}\right) @ 0=\lfloor\operatorname{LM}(v)\rfloor \quad \underline{\operatorname{LAM}}\left(v, M_{0}\right) @ n+1=M_{0} @ n \\
\underline{\operatorname{APP}}\left(M_{1}, M_{2}\right) @ 2 \cdot n+1=M_{1} @ n \quad \underline{\operatorname{APP}}\left(M_{1}, M_{2}\right) @ 0=\lfloor\operatorname{AP}\rfloor \\
\hline
\end{gathered}
$$

We note that node-observations completely characterize a $\lambda$-tree:

Lemma 31 If for all $n \in \omega, M @ n=M^{\prime} @ n$, then $M=M^{\prime}$.

Proof: By Lemma 16, it suffices to show that $\forall k .|M|^{k}=\left|M^{\prime}\right|^{k}$, by induction on $k$. The case $k=0$ is trivial: both sides are $\square$. For the inductive step, we have to show $|M|^{k+1}=\left|M^{\prime}\right|^{k+1}$ assuming the $k$ th cuts are equal. We proceed by cases on $M$ :

Case $M=\square$ : Then $M @ 0=\perp$, so by assumption, also $M^{\prime} @ 0=\perp$, and we must have $M^{\prime}=\square$. Hence, in particular, $|M|^{k+1}=\left|M^{\prime}\right|^{k+1}=\square$.

Case $M=\underline{\operatorname{VAR}}(v)$ : Then $M @ 0=\lfloor\operatorname{VR}(v)\rfloor$, so also $M^{\prime} @ 0=\lfloor\operatorname{VR}(v)\rfloor$, which can only happen if $M^{\prime}=\underline{\operatorname{VAR}}(v)$, so again we get $|M|^{k+1}=\left|M^{\prime}\right|^{k+1}=\underline{\operatorname{VAR}}(v)$.

Case $M=\underline{\operatorname{LAM}}\left(v, M_{0}\right)$ : Then $M @ 0=\lfloor\operatorname{LM}(v)\rfloor$, so also $M^{\prime} @ 0=\lfloor\operatorname{LM}(v)\rfloor$, which means that $M^{\prime}=\underline{\operatorname{LAM}}\left(v, M_{0}^{\prime}\right)$ for some $M_{0}^{\prime}$. Moreover, we have, for any $n$,

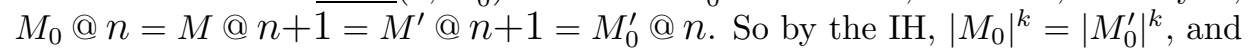
thus $|M|^{k+1}=\underline{\operatorname{LAM}}\left(v,\left|M_{0}\right|^{k}\right)=\underline{\operatorname{LAM}}\left(v,\left|M_{0}^{\prime}\right|^{k}\right)=\left|M^{\prime}\right|^{k+1}$.

Case $M=\underline{\operatorname{APP}}\left(M_{1}, M_{2}\right)$ : Then $M @ 0=\lfloor\mathrm{AP}\rfloor$, so also $M^{\prime} @ 0=\lfloor\mathrm{AP}\rfloor$, which means that $M^{\prime}=\underline{\mathrm{APP}}\left(M_{1}^{\prime}, M_{2}^{\prime}\right)$ for some $M_{1}^{\prime}$ and $M_{2}^{\prime}$. Now, for any $n, M_{1} @ n=M @$ $2 \cdot n+1=M^{\prime} @ 2 \cdot n+1=M_{1}^{\prime} @ n$, so by IH, $\left|M_{1}\right|^{k}=\left|M_{1}^{\prime}\right|^{k}$. Analogously, we get $\left|M_{2}\right|^{k}=\left|M_{2}^{\prime}\right|^{k}$, and thus $|M|^{k+1}=\underline{\operatorname{APP}}\left(\left|M_{1}\right|^{k},\left|M_{2}\right|^{k}\right)=\underline{\operatorname{APP}}\left(\left|M_{1}^{\prime}\right|^{k},\left|M_{2}^{\prime}\right|^{k}\right)=$ $\left|M^{\prime}\right|^{k+1}$. 


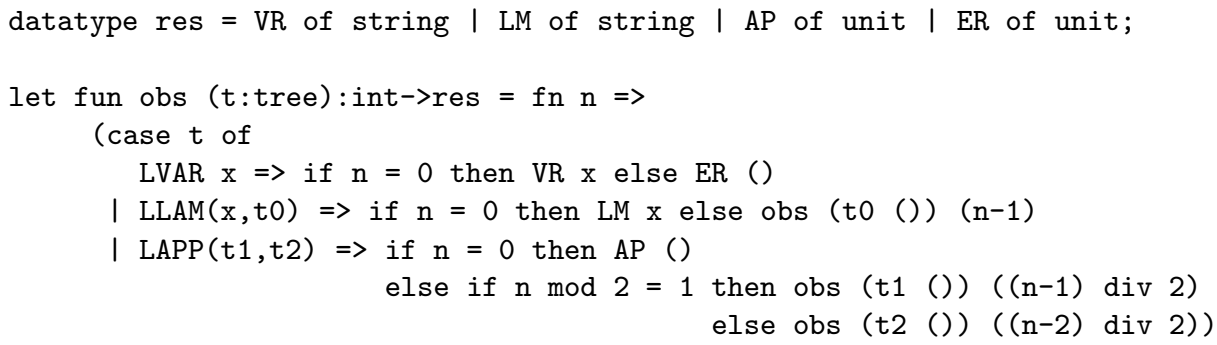

in obs end

Figure 5: A simple observation function, $O B S$.

\subsubsection{Implementing tree-observations in ML}

The ML implementation of the observation function is shown in Figure 5. To represent observation results, we introduce another ground datatype res; like for term, we assume that $\llbracket r e s \rrbracket^{\mathrm{ml}}=\mathrm{O}$, and that the meanings of the constructors agree. We also assume that the ML fragment has been extended with aritmetic operators - , div, and mod, completely analogous to the existing + .

Lemma 32 For all $M \in \underline{\Lambda}$ and $n \in \omega, M @ n=i_{\underline{\Lambda} T}(M) \star \lambda t . \theta_{\text {obs }} t \star \lambda f . f n$.

Proof: We first calculate, using that $\theta_{\text {obs }}=\operatorname{fix}\left(\Theta_{\text {obs }}\right)$, for any $t \in T$ :

$$
\begin{aligned}
& \theta_{\text {obs }} t \star \lambda f . f n \\
& =\Theta_{\text {obs }}\left(\theta_{\text {obs }}\right) t \star \lambda f . f n
\end{aligned}
$$

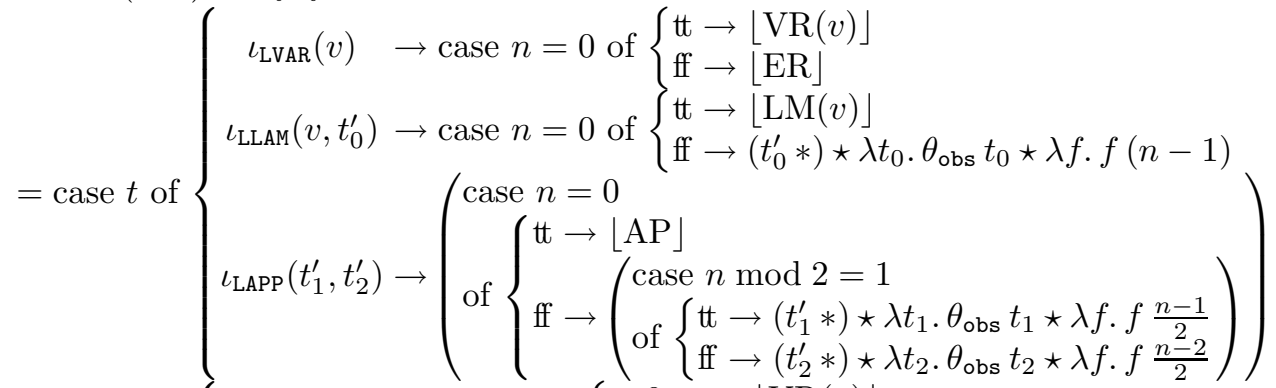

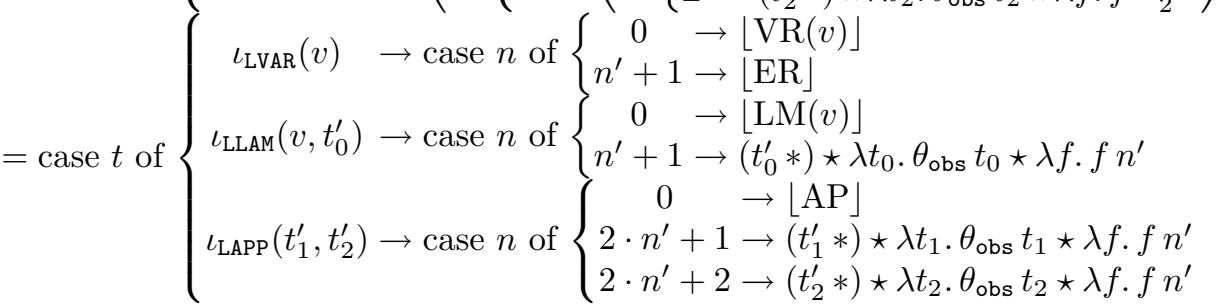

The proof then proceeds by a straightforward course-of-values induction on $n$. We assume the lemma holds for all $n^{\prime}<n$, and consider the four possibilities for $M$ :

When $M=\square$, both sides are $\perp$, by Lemma 26(a). Of the remaining cases, we show just $M=\underline{\operatorname{LAM}}\left(x, M_{0}\right)$ in detail: 


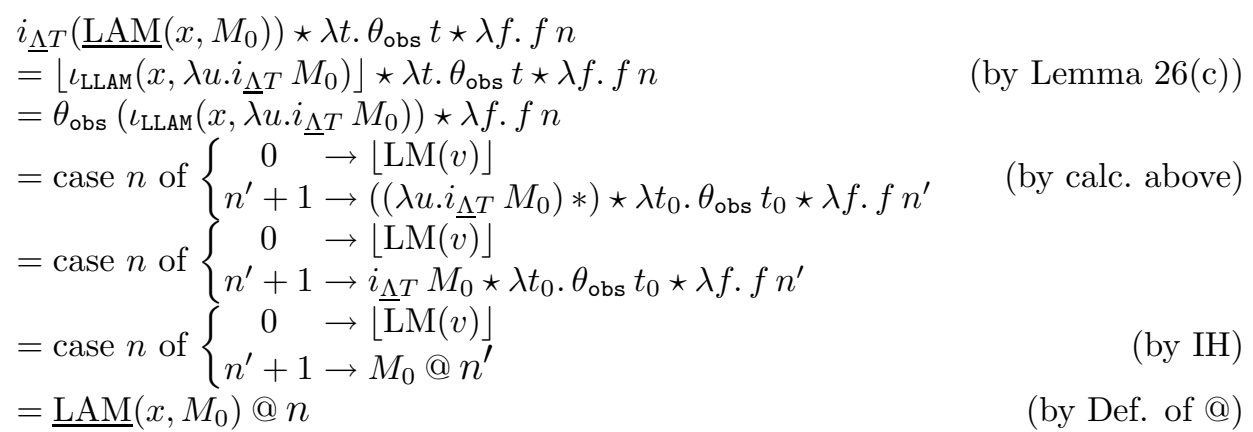

The cases for $M=\underline{\operatorname{VAR}}(v)$ and $M=\underline{\operatorname{APP}}\left(M_{1}, M_{2}\right)$ are completely analogous.

Consider now an ML program whose datatype declarations are a union of those in Figures 4 and 5 (in any order), and take

$O B S B T=$ fn $\mathrm{m} \Rightarrow O B S(B T \mathrm{~m})$.

This is an ML expression of type term $\rightarrow$ int $\rightarrow$ res, i.e., a complete program.

Theorem 7 (cf. Theorem 4) For all $m \in \Lambda, n \in \omega$, and $o \in \mathrm{O}, \mathrm{OBSBT} \bullet(m, n)=o$ iff bt $(m) @ n=\lfloor o\rfloor$.

Proof: We first note that, since $B T$ and $O B S$ are closed, for any $\xi, \llbracket B T \rrbracket^{\mathrm{ml}} \xi=\left\lfloor\theta_{\mathrm{bt}}\right\rfloor$ and $\llbracket O B S \rrbracket^{\mathrm{ml}} \xi=\left\lfloor\theta_{\text {obs }}\right\rfloor$. Then,

$$
\begin{aligned}
& \llbracket O B S B T \rrbracket^{\mathrm{ml}} \emptyset \star \lambda f_{1} \cdot f_{1} m \star \lambda f_{2} \cdot f_{2} n \\
& =\llbracket \mathrm{fn} \mathrm{m} \Rightarrow O B S(B T \mathrm{~m}) \rrbracket^{\mathrm{ml}} \emptyset \star \lambda f_{1} \cdot f_{1} m \star \lambda f_{2} \cdot f_{2} n \\
& =\llbracket O B S \quad(B T \mathrm{~m}) \rrbracket^{\mathrm{ml} \emptyset} \emptyset[\mathrm{m} \mapsto m] \star \lambda f_{2} . f_{2} n \\
& =\left(\theta_{\mathrm{bt}} m \star \lambda t . \theta_{\text {obs }} t\right) \star \lambda f_{2} . f_{2} n \\
& =\theta_{\mathrm{bt}} m \star \lambda t . \theta_{\mathrm{obs}} t \star \lambda f_{2} . f_{2} n \\
& =i_{\Lambda T}(\mathrm{bt}(m)) \star \lambda t . \theta_{\mathrm{obs}} t \star \lambda f_{2} . f_{2} n \\
& =\overline{\mathrm{bt}}(m) @ n
\end{aligned}
$$

The result then follows immediately from Theorem 3 .

Moreover, Lemma 31 tells us that $B T$ is also operationally correct with respect to any other observation function (including ones using more user-friendly access paths), because $O B S$-observations can already distinguish all elements of type tree, even those that do not represent proper Böhm trees.

\section{Conclusions and perspectives}

We have presented a domain-theoretic analysis of a normalization-by-evaluation construction for the untyped $\lambda$-calculus. Compared to the typed case, the main difference is a change from induction on types to general recursion, both for function definitions and for the domains and relations on them. That the correctness proof has a generalized computational-adequacy result at its core, further strengthens the connection between normalization and evaluation. Moreover, the algorithmic content of the construction corresponds very directly to a simple functional program, enabling a precise verification of the normalizer as actually implemented. 
There are several possible directions in which to extend or modify the present work. Especially in the infinitary variant of the algorithm, there is some leeway in exactly what kind of $\lambda$-trees we wish to consider as output, and our observation model for them. It should also be possible to extend the language and notion of normalization with interpreted constants in a suitable sense. But already the current results indicate that the fundamental ideas of NBE are not incompatible with general recursive types. Thus, reduction-free normalization may provide a complementary view of other equational systems that are currently analyzed using exclusively reduction-based methods. It might even be possible to find unified formulations of rewriting-theoretic and model-theoretic normalization results about particular such systems.

Acknowledgment The authors wish to thank Olivier Danvy, as well as the FOSSACS'04 and RAIRO-ITA reviewers, for their insightful comments. 


\section{A Existence of invariant relations}

For completeness, we review Pitts's technique. For conciseness, let us fix our attention to the recursive domain equation

$$
X \cong(A+[X \rightarrow X])_{\perp}
$$

where $A$ is a cpo.

A solution to this equation is a pointed cpo $D$ and an isomorphism $i: D \cong$ $(A+[D \rightarrow D])_{\perp}$. Define the continuous function $\delta:[D \rightarrow D] \rightarrow[D \rightarrow D]$ as

$$
\delta(e)(d)=\text { case } d \text { of }\left\{\begin{aligned}
i^{-1}\left(\left\lfloor i n_{1}(a)\right\rfloor\right) & \rightarrow i^{-1}\left(\left\lfloor i n_{1}(a)\right\rfloor\right) \\
i^{-1}\left(\left\lfloor i n_{2}(f)\right\rfloor\right) & \rightarrow i^{-1}\left(\left\lfloor i n_{2}(e \circ f \circ e)\right\rfloor\right) \\
\perp_{D} & \rightarrow \perp_{D}
\end{aligned}\right.
$$

A solution is called a minimal invariant if $\operatorname{fix}(\delta)=i d_{D}$.

The following is well-known and can be found in in e.g. Pitts [Pit96]:

Theorem 8 For any cpo $A$, there exists a minimal invariant to the recursive domain equation $X \cong(A+[X \rightarrow X])_{\perp}$.

This section establishes the following result, which is an abstract version of the construction used by Pitts to show computational adequacy for untyped PCF [Pit93]:

Theorem 1 Let $A$ be a cpo, and let $i: D \cong(A+[D \rightarrow D])_{\perp}$ be a minimal invariant. Let $T$ be a set, and let predicates $P_{1} \subseteq A \times T, P_{2} \subseteq T$, and $P_{3} \subseteq T \times T \times T$ be given, such that $\left\{a \mid P_{1}(a, t)\right\}$ is inclusive for every $t \in T$. Then there exists a relation $\triangleleft \subseteq D \times T$, with $\{d \mid d \triangleleft t\}$ inclusive for every $t \in T$, such that, for all $d \in D$ and $t \in T$,

$$
d \triangleleft t i f f\left(\begin{array}{l}
d=\perp_{D} \vee \\
\exists a \cdot d=i^{-1}\left(\left\lfloor i n_{1}(a)\right\rfloor\right) \wedge P_{1}(a, t) \vee \\
\exists f . d=i^{-1}\left(\left\lfloor i n_{2}(f)\right\rfloor\right) \wedge P_{2}(t) \wedge \\
\forall d^{\prime} \in D ; t^{\prime}, t^{\prime \prime} \in T . P_{3}\left(t, t^{\prime}, t^{\prime \prime}\right) \wedge d^{\prime} \triangleleft t^{\prime} \Rightarrow f\left(d^{\prime}\right) \triangleleft t^{\prime \prime}
\end{array}\right) .
$$

To show the theorem, let $A,(D, i)$, and $T$ be given. Define a set Rel of relations on $D \times T$ by

$R \in R e l$ iff for all $t \in T,\{d \mid(d, t) \in R\}$ is a pointed, inclusive subset of $D$.

Then $(R e l, \subseteq)$ is a partial order, where $\subseteq$ is ordinary set inclusion. Since Rel is closed under arbitrary intersection, $(R e l, \subseteq)$ is in fact a complete lattice. (Note, however, that joins in this lattice are not in general set-theoretic unions, since the union of an arbitrary family of inclusive relations need not itself be inclusive. Rather, $\bigsqcup\left\{R_{i} \mid i \in I\right\}=\bigcap\left\{R \in \operatorname{Rel} \mid \forall i \in I . R_{i} \subseteq R\right\}$, i.e., the smallest inclusive relation containing all of the $R_{i}$.) In particular, $R e l^{\mathrm{op}}$, i.e., $R e l$ ordered by $\supseteq$, is also a complete lattice, and so is $\operatorname{Rel}^{\mathrm{op}} \times$ Rel. 
Now, let predicates $P_{1} \subseteq A \times T, P_{2} \subseteq T$, and $P_{3} \subseteq T \times T \times T$ be given, with $P_{1}(\cdot, t)$ inclusive for all $t \in T$. Define $\mathcal{R}: R e l^{\mathrm{op}} \times \operatorname{Rel} \rightarrow$ Rel by

$$
\begin{aligned}
& \mathcal{R}\left(R^{-}, R^{+}\right)= \\
& \left\{\begin{array}{l|l}
(d, t) & \begin{array}{l}
d=\perp_{D} \vee \\
\exists a . d=i^{-1}\left(\left\lfloor i n_{1}(a)\right\rfloor\right) \wedge P_{1}(a, t) \vee \\
\exists f . d=i^{-1}\left(\left\lfloor i n_{2}(f)\right\rfloor\right) \wedge P_{2}(t) \wedge \\
\forall d^{\prime} \in D ; t^{\prime}, t^{\prime \prime} \in T . P_{3}\left(t, t^{\prime}, t^{\prime \prime}\right) \wedge\left(d^{\prime}, t^{\prime}\right) \in R^{-} \Rightarrow\left(f\left(d^{\prime}\right), t^{\prime \prime}\right) \in R^{+}
\end{array}
\end{array}\right\} .
\end{aligned}
$$

$\mathcal{R}$ is clearly monotone. It is also easy to check that it preserves inclusivity: in addition to the usual closure under abitrary intersection, finite union, and inverse image, we use that the two existentials are guarded by order-monics, so that, e.g., in the second disjunct, a chain $d_{1} \sqsubseteq d_{2} \sqsubseteq \cdots$ also induces a chain $a_{1} \sqsubseteq a_{2} \sqsubseteq \cdots$, allowing us to exploit inclusivity of $P_{1}(\cdot, t)$. To prove Theorem 1 , we thus only need to show that there exists a relation $\triangleleft \in \operatorname{Rel}$ such that $\triangleleft=\mathcal{R}(\triangleleft, \triangleleft)$. We first establish a seemingly weaker result:

Lemma 33 There exist relations $\triangleleft^{-}, \triangleleft^{+} \in$ Rel, satisfying:

a. $\triangleleft^{-}=\mathcal{R}\left(\triangleleft^{+}, \triangleleft^{-}\right)$and $\triangleleft^{+}=\mathcal{R}\left(\triangleleft^{-}, \triangleleft^{+}\right)$.

b. For all $R^{-}, R^{+} \in$ Rel, if $R^{-} \subseteq \mathcal{R}\left(R^{+}, R^{-}\right)$and $\mathcal{R}\left(R^{-}, R^{+}\right) \subseteq R^{+}$, then $R^{-} \subseteq$ $\triangleleft^{-}$and $\triangleleft^{+} \subseteq R^{+}$.

Proof: Define the symmetric extension of $\mathcal{R}, \widehat{\mathcal{R}}: \operatorname{Re} l^{\mathrm{op}} \times \operatorname{Rel} \rightarrow \operatorname{Re} l^{\mathrm{op}} \times \operatorname{Rel}$, by

$$
\widehat{\mathcal{R}}\left(R^{-}, R^{+}\right)=\left(\mathcal{R}\left(R^{+}, R^{-}\right), \mathcal{R}\left(R^{-}, R^{+}\right)\right) .
$$

Now $\widehat{\mathcal{R}}$ is a monotonic operator on a complete lattice, so by the Knaster-Tarski fixed-point theorem, $\widehat{\mathcal{R}}$ has a fixed point $\left(\triangleleft^{-}, \triangleleft^{+}\right)$that is also the least prefixed point of $\widehat{\mathcal{R}}$. That is, we have (a) $\left(\triangleleft^{-}, \triangleleft^{+}\right)=\widehat{\mathcal{R}}\left(\triangleleft^{-}, \triangleleft^{+}\right)$, and (b) if $\widehat{\mathcal{R}}\left(R^{-}, R^{+}\right) \sqsubseteq R e l^{\text {op }} \times \operatorname{Rel}$ $\left(R^{-}, R^{+}\right)$then $\left(\triangleleft^{-}, \triangleleft^{+}\right) \sqsubseteq R e l^{\mathrm{op}} \times \operatorname{Rel}\left(R^{-}, R^{+}\right)$. And these are precisely the properties claimed in the statement of the lemma.

For relations $R, S \in R e l$, we now define a predicate on $e \in[D \rightarrow D]$ by:

$$
e: R \subset S \text { iff } \forall d \in D, t \in T .(d, t) \in R \Rightarrow(e(d), t) \in S
$$

Since this predicate is defined as an intersection of inverse images of the inclusive $S$, it is itself inclusive.

Lemma 34 If $e: R \subset S$ then $\delta(e): \mathcal{R}(S, R) \subset \mathcal{R}(R, S)$.

Proof: Assume $e: R \subset S$, and let $(d, t) \in \mathcal{R}(S, R)$ be given; we must show that $(\delta(e)(d), t) \in \mathcal{R}(R, S)$. Consider $d$. The cases $d=\perp_{D}$ and $d=i^{-1}\left(\left\lfloor i n_{1}(l)\right\rfloor\right)$ do not depend on $R, S$, or $e$, and are thus immediate. Assume now $d=i^{-1}\left(\left\lfloor i n_{2}(f)\right\rfloor\right)$ where by assumption, $P_{2}(t)$ and $\forall d^{\prime}, t^{\prime}, t^{\prime \prime} . P_{3}\left(t, t^{\prime}, t^{\prime \prime}\right) \wedge\left(d^{\prime}, t^{\prime}\right) \in S \Rightarrow\left(f\left(d^{\prime}\right), t^{\prime \prime}\right) \in R$. Then $\delta(e)(d)=i^{-1}\left(\left\lfloor i n_{2}(e \circ f \circ e)\right\rfloor\right)$. $P_{2}(t)$ still holds. Let $d^{\prime}, t^{\prime}, t^{\prime \prime}$ be given, such that $P_{3}\left(t, t^{\prime}, t^{\prime \prime}\right) \wedge\left(d^{\prime}, t^{\prime}\right) \in R$; we must show $\left.(e \circ f \circ e)\left(d^{\prime}\right), t^{\prime \prime}\right) \in S$. We calculate: by $e: R \subset S,\left(e\left(d^{\prime}\right), t^{\prime}\right) \in S$; by assumption on $f,\left(f\left(e\left(d^{\prime}\right)\right), t^{\prime \prime}\right) \in R$; and by $e: R \subset S$ again, $\left(e\left(f\left(e\left(d^{\prime}\right)\right)\right), t^{\prime \prime}\right) \in S$, as required. 
Finally, we can show that $\triangleleft^{-}$and $\triangleleft^{+}$are in fact the same relation:

Lemma 35 The relations $\triangleleft^{-}$and $\triangleleft^{+}$are equal.

Proof: We show that each relation is included in the other. First, take $R^{-}=\triangleleft^{+}$ and $R^{+}=\triangleleft^{-}$. By Lemma 33(a) we then get that $R^{+}=\mathcal{R}\left(R^{-}, R^{+}\right)$and $R^{-}=$ $\mathcal{R}\left(R^{+}, R^{-}\right)$. Hence, by Lemma 33(b) (either half), $\triangleleft^{+} \subseteq \triangleleft^{-}$.

Conversely, we have by Lemma 33(a) and Lemma 34 that if $e: \triangleleft^{-} \subset \triangleleft^{+}$then $\delta(e): \triangleleft^{-} \subset \triangleleft^{+}$. Since $(\perp, t) \in \triangleleft^{+}$for any $t$, we also have $\perp_{[D \rightarrow D]}: \triangleleft^{-} \subset \triangleleft^{+}$. Thus, by fixed-point induction, fix $(\delta): \triangleleft^{-} \subset \triangleleft^{+}$. And since $(D, i)$ is a minimal invariant, fix $(\delta)=i d_{D}$, and so $i d_{D}: \triangleleft^{-} \subset \triangleleft^{+}$, i.e. $\triangleleft^{-} \subseteq \triangleleft^{+}$.

Taking $\triangleleft=\triangleleft^{+}=\triangleleft^{-}$, and using Lemma 33(a) (either half), we have thus established $\triangleleft=\mathcal{R}(\triangleleft, \triangleleft)$, and hence proven Theorem 1 .

\section{B Existence of isomorphisms}

In the following, we show that the recursive domains used in the abstract normalization constructions, correspond naturally to the predomains arising as the denotations of recursive types in the ML implementation. Naturally, the two specific instances covered here are examples of a more general correspondance, essentially building on currying/uncurrying isomorphisms; however, setting up a proper framework for obtaining such correspondences uniformly, would be too tangential to the paper's main topic of NBE constructions.

\section{B.1 Isomorphisms for the redualizing model}

In appendix $\mathrm{A}$, we considered minimal-invariant solutions $(D, i)$ of the recursive domain equation,

$$
X \cong(A+[X \rightarrow X])_{\perp},
$$

where $A$ was some fixed cpo. Similarly, we may consider the recursive predomain equation,

$$
Y \cong B+\left[\left[\mathbf{1} \rightarrow Y_{\perp}\right] \rightarrow Y_{\perp}\right],
$$

for some cpo $B$. A solution to this equation consists of a (bottomless) cpo $S$ and an isomorphism $j: S \cong B+\left[\left[\mathbf{1} \rightarrow S_{\perp}\right] \rightarrow S_{\perp}\right]$. Define the continuous function $\gamma:\left[S \rightarrow S_{\perp}\right] \rightarrow\left[S \rightarrow S_{\perp}\right]$ by

$$
\gamma(e)(s)=\text { case } s \text { of }\left\{\begin{array}{l}
j^{-1}\left(i n_{1}(a)\right) \rightarrow\left\lfloor j^{-1}\left(i n_{1}(a)\right)\right\rfloor \\
j^{-1}\left(i n_{2}(f)\right) \rightarrow\left\lfloor j^{-1}\left(i n_{2}\left(\lambda t^{1 \rightarrow S_{\perp}} . f(\lambda u .(t *) \star e) \star e\right)\right)\right\rfloor
\end{array}\right.
$$

A solution is called a minimal invariant if $\operatorname{fix}(\gamma)=\lambda s$. $\lfloor s\rfloor$. The standard inverse-limit construction, re-expressed in the setting of predomains and total continuous functions, finds minimal-invariant solutions in this sense.

We will also need the following simple property about fixed points:

Lemma 36 Let $D$ and $E$ be pointed cpos, and let $f: D \rightarrow D$ and $g: E \rightarrow E$ be continuous functions. If $c: D \rightarrow E$ is a strict continuous function such that $c \circ f=g \circ c$, then $c(\operatorname{fix}(f))=\operatorname{fix}(g)$. 
Proof: By fixed point induction. Define the admissible predicate $P(d, e) \Leftrightarrow c(d)=e$ as an inverse image of the equality predicate. Since $c$ is strict, we have $P\left(\perp_{D}, \perp_{E}\right)$ and so $P$ is also pointed. Let now $d$ and $e$ be given such that $P(d, e)$, i.e., $c(d)=e$. By assumption on $f$ and $g$, also $c(f(d))=g(c(d))=g(e)$, i.e., $P(f(d), g(e))$. Thus by the continuity of $f$ and $g, P(\operatorname{fix}(f)$, fix $(g))$ or simply $c(\operatorname{fix}(f))=\operatorname{fix}(g)$.

We are now in a position to establish the existence of isomorphisms between domains and predomains from minimal invariants for the above equations.

Lemma 37 Let $A$ and $B$ be isomorphic cpos via $i_{A B}: A \cong B$; let $(D, i)$ be a minimal invariant for the recursive domain equation $X \cong(A+[X \rightarrow X])_{\perp}$; and let $(S, j)$ be a minimal invariant for the recursive predomain equation $Y \cong B+\left[\left[\mathbf{1} \rightarrow Y_{\perp}\right] \rightarrow Y_{\perp}\right]$. Then there exists an isomorphism $i_{D S}: D \cong S_{\perp}$, satisfying

a. For all $a \in A, i_{D S}\left(i^{-1}\left(\left\lfloor i n_{1}(a)\right\rfloor\right)\right)=\left\lfloor j^{-1}\left(i n_{1}\left(i_{A B}(a)\right)\right)\right\rfloor$.

b. For all $f \in[D \rightarrow D]$,

$$
i_{D S}\left(i^{-1}\left(\left\lfloor i n_{2}(f)\right\rfloor\right)\right)=\left\lfloor j^{-1}\left(i n_{2}\left(\lambda t^{\mathbf{1} \rightarrow S_{\perp}} \cdot i_{D S}\left(f\left(i_{D S}^{-1}(t *)\right)\right)\right)\right)\right\rfloor .
$$

c. $i_{D S}\left(\perp_{D}\right)=\perp_{S_{\perp}}$.

Proof: By direct construction. For any strict functions $h: D \rightarrow S_{\perp}$ and $k: S_{\perp} \rightarrow D$, define the strict $H(h, k): D \rightarrow S_{\perp}$ and $K(h, k): S_{\perp} \rightarrow D$ by

$$
\begin{aligned}
& H(h, k)=\lambda d \text {.case } d \text { of }\left\{\begin{aligned}
i^{-1}\left(\left\lfloor i n_{1}(a)\right\rfloor\right) & \rightarrow\left\lfloor j^{-1}\left(i n_{1}\left(i_{A B}(a)\right)\right)\right\rfloor \\
i^{-1}\left(\left\lfloor i n_{2}(f)\right\rfloor\right) & \rightarrow\left\lfloor j^{-1}\left(i n_{2}\left(\lambda t^{1 \rightarrow S_{\perp}} . h(f(k(t *)))\right)\right)\right\rfloor \\
\perp_{D} & \rightarrow \perp_{S_{\perp}}
\end{aligned}\right. \\
& K(h, k)=\lambda s^{\prime} \cdot s^{\prime} \star \lambda s . \text { case } s \text { of }\left\{\begin{array}{l}
j^{-1}\left(i n_{1}(b)\right) \rightarrow i^{-1}\left(\left\lfloor i n_{1}\left(i_{A B}^{-1}(b)\right)\right\rfloor\right) \\
j^{-1}\left(i n_{2}(g)\right) \rightarrow i^{-1}\left(\left\lfloor i n_{2}\left(\lambda d . k\left(g\left(\lambda u^{\mathbf{1}} . h d\right)\right)\right)\right\rfloor\right)
\end{array}\right.
\end{aligned}
$$

Then define $\left(i_{D S}, i_{D S}^{-1}\right)=\operatorname{fix}\left(\lambda(h, k)^{\left[D \rightarrow S_{\perp}\right] \times\left[S_{\perp} \rightarrow D\right]} .(H(h, k), K(h, k))\right)$.

We need to show that $i_{D S}$ and $i_{D S}^{-1}$ are in fact two-sided inverses. Let $c$ be the strict function $\lambda(h, k) . k \circ h:\left[D \rightarrow S_{\perp}\right] \times\left[S_{\perp} \rightarrow D\right] \rightarrow[D \rightarrow D]$. Now,

$$
\begin{aligned}
& (c \circ \lambda(h, k) .(H(h, k), K(h, k)))(h, k) \\
& =K(h, k) \circ H(h, k) \\
& \left\{i^{-1}\left(i n_{1}(a)\right) \rightarrow K(h, k)\left(\left\lfloor j^{-1}\left(i n_{1}\left(i_{A B}(a)\right)\right)\right\rfloor\right)\right.
\end{aligned}
$$

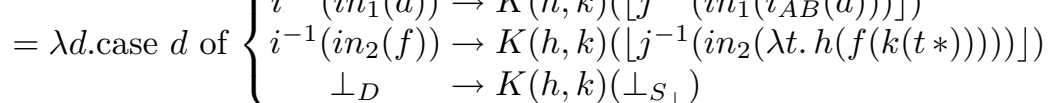

$$
\begin{aligned}
& =\lambda d \text {.case } d \text { of }\left\{\begin{aligned}
\perp_{D} & \rightarrow K(h, k)\left(\perp_{S_{\perp}}\right) \\
i^{-1}\left(i_{1}(a)\right) & \rightarrow i^{-1}\left(\left\lfloor i n_{1}\left(i_{A B}^{-1}\left(i_{A B}(a)\right)\right)\right\rfloor\right) \\
\perp_{D} & \rightarrow i^{-1}\left(\left\lfloor i n_{2}(\lambda d . k((\lambda t . h(f(k(t *))))(\lambda u . h d)))\right\rfloor\right) \\
& \rightarrow \perp_{D}
\end{aligned}\right. \\
& =\lambda d . \text { case } d \text { of }\left\{\begin{aligned}
i^{-1}\left(i n_{1}(a)\right) & \rightarrow i^{-1}\left(\left\lfloor i n_{1}(a)\right\rfloor\right) \\
i^{-1}\left(i n_{2}(f)\right) & \rightarrow i^{-1}\left(\left\lfloor i n_{2}(k \circ h \circ f \circ k \circ h)\right\rfloor\right) \\
\perp_{D} & \rightarrow \perp_{D}
\end{aligned}\right.
\end{aligned}
$$

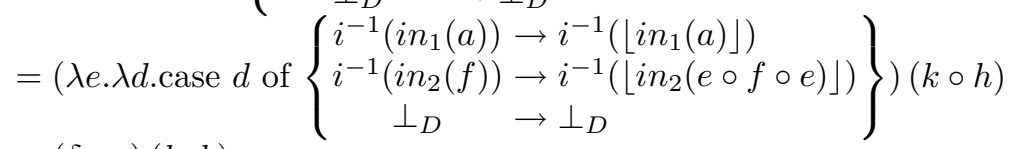

$$
\begin{aligned}
& =(\delta \circ c)(h, k)
\end{aligned}
$$

Hence, by Lemma 36 and the minimal invariant property of $(D, i)$,

$$
i_{D S}^{-1} \circ i_{D S}=c(\operatorname{fix}(\lambda(h, k) .(H(h, k), K(h, k))))=\operatorname{fix}(\delta)=i d_{D} .
$$


For the other direction, let $c^{\prime}$ be the strict function $\lambda(h, k) . h \circ k \circ(\lambda s .\lfloor s\rfloor)$ : $\left[D \rightarrow S_{\perp}\right] \times\left[S_{\perp} \rightarrow D\right] \rightarrow\left[S \rightarrow S_{\perp}\right]$. We proceed similarly,

$\left(c^{\prime} \circ \lambda(h, k) \cdot(H(h, k), K(h, k))\right)(h, k)$

$=H(h, k) \circ K(h, k) \circ(\lambda s .\lfloor s\rfloor)$

$=\lambda s$. case $s$ of $\left\{\begin{array}{l}j^{-1}\left(i n_{1}(b)\right) \rightarrow H(h, k)\left(i^{-1}\left(\left\lfloor i n_{1}\left(i_{A B}^{-1}(b)\right)\right\rfloor\right)\right) \\ j^{-1}\left(i n_{2}(g)\right) \rightarrow H(h, k)\left(i^{-1}\left(\left\lfloor i n_{2}(\lambda d . k(g(\lambda u . h d)))\right\rfloor\right)\right)\end{array}\right.$

$=\lambda$ s.case $s$ of $\left\{j^{-1}\left(i n_{1}(b)\right) \rightarrow\left\lfloor j^{-1}\left(i n_{1}\left(i_{A B}\left(i_{A B}^{-1}(b)\right)\right)\right)\right\rfloor\right.$

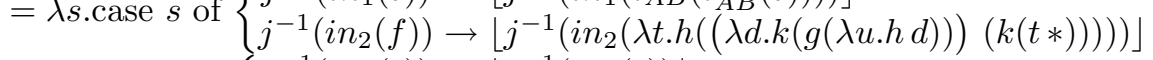

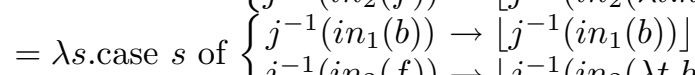

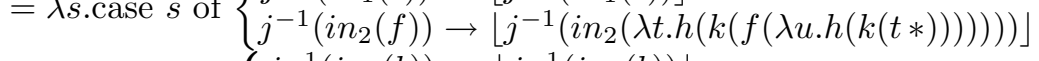

$=\lambda s$. case $s$ of $\left\{\begin{array}{l}j^{-1}\left(i n_{1}(b)\right) \rightarrow\left\lfloor j^{-1}\left(i n_{1}(b)\right)\right\rfloor \\ j^{-1}\left(i n_{2}(f)\right) \rightarrow\left\lfloor j^{-1}\left(i n_{2}\left(\lambda t \cdot\left(\begin{array}{c}f(\lambda u \cdot(t *) \star \lambda s \cdot h(k(\lfloor s\rfloor))) \star \\ \lambda s \cdot h(k(\lfloor s\rfloor))\end{array}\right)\right)\right\rfloor\right.\end{array}\right.$

$=\left(\lambda e . \lambda s\right.$. case $s$ of $\left.\left\{\begin{array}{l}j^{-1}\left(i n_{1}(b)\right) \rightarrow\left\lfloor j^{-1}\left(i n_{1}(b)\right)\right\rfloor \\ j^{-1}\left(i n_{2}(f)\right) \rightarrow\left\lfloor j^{-1}\left(i n_{2}(\lambda t . f(\lambda u .(t *) \star e) \star e)\right)\right\rfloor\end{array}\right\}\right)$

$(\lambda \operatorname{s.h}(k(\lfloor s\rfloor)))$

$=\left(\gamma \circ c^{\prime}\right)(h, k)$

By Lemma 36 and the minimal-invariant property of $(S, j)$,

$$
i_{D S} \circ i_{D S}^{-1} \circ(\lambda s .\lfloor s\rfloor)=c^{\prime}(\operatorname{fix}(\lambda(h, k) .(H(h, k), K(h, k))))=\operatorname{fix}(\gamma)=\lambda s .\lfloor s\rfloor .
$$

Also, $i_{D S}\left(i_{D S}^{-1}(\perp)\right)=\perp$ by strictness of $i_{D S}$ and $i_{D S}^{-1}$. Thus, $i_{D S}: D \cong S_{\perp}$ is indeed an isomorphism. From the fixed point equation $i_{D S}=H\left(i_{D S}, i_{D S}^{-1}\right)$, we can then immediately read off the additional properties in parts $(\mathrm{a}-\mathrm{c})$ of the Lemma.

Lemma 37 in particular establishes Lemma 12, taking $A=B=\left[\mathbb{Z} \rightarrow \Lambda_{\perp}\right]$ and $i_{A B}=i d_{\left[\mathbb{Z} \rightarrow \Lambda_{\perp}\right]}$. It also establishes Lemma 28 , with $A=[\mathbb{Z} \rightarrow \underline{\Lambda}], B=\left[\mathbb{Z} \rightarrow T_{\perp}\right]$, and $i_{A B}=\lambda l . \lambda n . i_{\underline{\Lambda} T}(l n)$, where $i_{\underline{\Lambda} T}: \underline{\Lambda} \stackrel{\cong}{\Rightarrow} T_{\perp}$ is the isomorphism from Lemma 26 .

\section{B.2 Isomorphisms for Böhm trees}

The proof of the existence of isomorphisms for Böhm trees proceeds analogously with above. For a cpo $A$, a solution to the recursive domain equation,

$$
X \cong(A+A \times X+X \times X)_{\perp}
$$

is a pointed cpo $D$ and an isomorphism $i: D \cong(A+A \times D+D \times D)_{\perp}$. The corresponding $\delta:[D \rightarrow D] \rightarrow[D \rightarrow D]$ is given by,

$$
\delta(e)(d)=\text { case } d \text { of }\left\{\begin{array}{cl}
i^{-1}\left(\left\lfloor i n_{1}(a)\right\rfloor\right) & \rightarrow i^{-1}\left(\left\lfloor i n_{1}(a)\right\rfloor\right) \\
i^{-1}\left(\left\lfloor i n_{2}\left(a, d_{0}\right)\right\rfloor\right) & \rightarrow i^{-1}\left(\left\lfloor i n_{2}\left(a, e\left(d_{0}\right)\right)\right\rfloor\right) \\
i^{-1}\left(\left\lfloor i n_{3}\left(d_{1}, d_{2}\right)\right\rfloor\right) & \rightarrow i^{-1}\left(\left\lfloor i n_{3}\left(e\left(d_{1}\right), e\left(d_{2}\right)\right)\right\rfloor\right) \\
\perp_{D} & \rightarrow \perp_{D}
\end{array}\right.
$$

Again, a solution is a minimal invariant if $\operatorname{fix}(\delta)=i d_{D}$.

Similarly, the recursive predomain equation,

$$
Y \cong A+A \times\left[\mathbf{1} \rightarrow Y_{\perp}\right]+\left[\mathbf{1} \rightarrow Y_{\perp}\right] \times\left[\mathbf{1} \rightarrow Y_{\perp}\right]
$$


has a minimal-invariant solution consisting of a (bottomless) cpo $S$ and an isomorphism $j: S \stackrel{\cong}{\rightarrow} A+A \times\left[\mathbf{1} \rightarrow S_{\perp}\right]+\left[\mathbf{1} \rightarrow S_{\perp}\right] \times\left[\mathbf{1} \rightarrow S_{\perp}\right]$, such that the continuous function $\gamma:\left[S \rightarrow S_{\perp}\right] \rightarrow\left[S \rightarrow S_{\perp}\right]$ given by

$$
\gamma(e)(s)=\text { case } s \text { of }\left\{\begin{aligned}
j^{-1}\left(i n_{1}(a)\right) & \rightarrow\left\lfloor j^{-1}\left(i n_{1}(a)\right)\right\rfloor \\
j^{-1}\left(i n_{2}\left(a, t_{0}\right)\right) & \rightarrow\left\lfloor j^{-1}\left(i n_{2}\left(a, \lambda u .\left(t_{0} *\right) \star e\right)\right)\right\rfloor \\
j^{-1}\left(i n_{3}\left(t_{1}, t_{2}\right)\right) & \rightarrow\left\lfloor j^{-1}\left(i n_{3}\left(\lambda u .\left(t_{1} *\right) \star e, \lambda u .\left(t_{2} *\right) \star e\right)\right)\right\rfloor
\end{aligned}\right.
$$

satisfies $\operatorname{fix}(\gamma)=\lambda s .\lfloor s\rfloor$.

Lemma 38 Let $A$ a cpo, let $(D, i)$ be a minimal invariant for the recursive domain equation $X \cong(A+A \times X+X \times X)_{\perp}$, and let $(S, j)$ be a minimal invariant for the recursive predomain equation $Y \cong A+A \times\left[\mathbf{1} \rightarrow Y_{\perp}\right]+\left[\mathbf{1} \rightarrow Y_{\perp}\right] \times\left[\mathbf{1} \rightarrow Y_{\perp}\right]$. Then there exists an isomorphism $i_{D S}: D \stackrel{\cong}{\rightrightarrows} S_{\perp}$, satisfying

a. $i_{D S}\left(\perp_{D}\right)=\perp_{S_{\perp}}$.

b. For all $a, i_{D S}\left(i^{-1}\left(\left\lfloor i n_{1}(a)\right\rfloor\right)\right)=\left\lfloor j^{-1}\left(i n_{1}(a)\right)\right\rfloor$.

c. For all $a$ and $d, i_{D S}\left(i^{-1}\left(\left\lfloor i n_{2}\left(a, d_{0}\right)\right\rfloor\right)=\left\lfloor j^{-1}\left(i n_{2}\left(a, \lambda u^{\mathbf{1}} \cdot i_{D S}\left(d_{0}\right)\right)\right)\right\rfloor\right.$.

d. For all $d_{1}$ and $d_{2}$,

$$
i_{D S}\left(i^{-1}\left(\left\lfloor i n_{3}\left(d_{1}, d_{2}\right)\right\rfloor\right)\right)=\left\lfloor j^{-1}\left(i n_{3}\left(\lambda u^{\mathbf{1}} . i_{D S}\left(d_{1}\right), \lambda u^{\mathbf{1}} \cdot i_{D S}\left(d_{2}\right)\right)\right)\right\rfloor .
$$

Proof: By direct construction. For any strict functions $h: D \rightarrow S_{\perp}$ and $k: S_{\perp} \rightarrow D$, define the strict $H(h): D \rightarrow S_{\perp}$ and $K(k): S_{\perp} \rightarrow D$ by

$$
\begin{gathered}
H(h)=\lambda d . \text { case } d \text { of }\left\{\begin{array}{cl}
i^{-1}\left(\left\lfloor i n_{1}(a)\right\rfloor\right) & \rightarrow\left\lfloor j^{-1}\left(i n_{1}(a)\right)\right\rfloor \\
i^{-1}\left(\left\lfloor i n_{2}\left(a, d_{0}\right)\right\rfloor\right) & \rightarrow\left\lfloor j^{-1}\left(i n_{2}\left(a, \lambda u \cdot h\left(d_{0}\right)\right)\right)\right\rfloor \\
i^{-1}\left(\left\lfloor i n_{3}\left(d_{1}, d_{2}\right)\right\rfloor\right) & \rightarrow\left\lfloor j^{-1}\left(i n_{3}\left(\lambda u \cdot h\left(d_{1}\right), \lambda u \cdot h\left(d_{2}\right)\right)\right)\right\rfloor \\
\perp_{D} & \rightarrow \perp_{\perp}
\end{array}\right. \\
K(k)=\lambda s^{\prime} \cdot s^{\prime} \star \lambda s . \text { case } s \text { of }\left\{\begin{array}{cc}
j^{-1}\left(i n_{1}(a)\right) & \rightarrow i^{-1}\left(\left\lfloor i n_{1}(a)\right\rfloor\right) \\
j^{-1}\left(i n_{2}\left(a, t_{0}\right)\right) & \rightarrow i^{-1}\left(\left\lfloor i n_{2}\left(a, k\left(t_{0} *\right)\right)\right\rfloor\right) \\
j^{-1}\left(i n_{3}\left(t_{1}, t_{2}\right)\right) & \rightarrow i^{-1}\left(\left\lfloor i n_{3}\left(k\left(t_{1} *\right), k\left(t_{2} *\right)\right)\right\rfloor\right)
\end{array}\right.
\end{gathered}
$$

Then define $\left(i_{D S}, i_{D S}^{-1}\right)=\operatorname{fix}\left(\lambda(h, k)^{\left[D \rightarrow S_{\perp}\right] \times\left[S_{\perp} \rightarrow D\right]} \cdot(H(h), K(k))\right)$.

Again, we need to show that $i_{D S}$ and $i_{D S}^{-1}$ are in fact two-sided inverses. Let $c$ be the strict function $\lambda(h, k) . k \circ h:\left[D \rightarrow S_{\perp}\right] \times\left[S_{\perp} \rightarrow D\right] \rightarrow[D \rightarrow D]$. Now,

$$
\begin{aligned}
& (c \circ \lambda(h, k) .(H(h), K(k)))(h, k) \\
& =K(k) \circ H(h) \\
& =\lambda d \text {.case } d \text { of }\left\{\begin{array}{cl}
i^{-1}\left(\left\lfloor i n_{1}(a)\right\rfloor\right) & \rightarrow i^{-1}\left(\left\lfloor i n_{1}(a)\right\rfloor\right) \\
i^{-1}\left(\left\lfloor i n_{2}\left(a, d_{0}\right)\right\rfloor\right) & \rightarrow i^{-1}\left(\left\lfloor i n_{2}\left(a, k\left(\left(\lambda u . h\left(d_{0}\right)\right) *\right)\right\rfloor\right)\right. \\
i^{-1}\left(\left\lfloor i n_{3}\left(d_{1}, d_{2}\right)\right\rfloor\right) & \rightarrow i^{-1}\left(\left\lfloor i n_{3}\left(k\left(\left(\lambda u \cdot h\left(d_{1}\right)\right) *\right), k\left(\left(\lambda u . h\left(d_{2}\right)\right) *\right)\right)\right\rfloor\right) \\
\perp_{D} & \rightarrow \perp_{D} \\
i^{-1}\left(\left\lfloor i n_{1}(a)\right\rfloor\right) & \rightarrow i^{-1}\left(\left\lfloor i n_{1}(a)\right\rfloor\right) \\
i^{-1}\left(\left\lfloor i n_{2}\left(a, d_{0}\right)\right\rfloor\right) & \rightarrow i^{-1}\left(\left\lfloor i n_{2}\left(a,(k \circ h)\left(d_{0}\right)\right)\right\rfloor\right) \\
i^{-1}\left(\left\lfloor i n_{3}\left(d_{1}, d_{2}\right)\right\rfloor\right) & \rightarrow i^{-1}\left(\left\lfloor i n_{3}\left((k \circ h)\left(d_{1}\right),(k \circ h)\left(d_{2}\right)\right)\right\rfloor\right) \\
\perp_{D} & \rightarrow \perp_{D}
\end{array}\right.
\end{aligned}
$$

By Lemma 36 and the minimal invariant property of $(D, i)$,

$$
i_{D S}^{-1} \circ i_{D S}=c(\operatorname{fix}(\lambda(h, k) .(H(h), K(k))))=\operatorname{fix}(\delta)=i d_{D} .
$$


For the other direction, let $c^{\prime}$ be the strict function $\lambda(h, k) . h \circ k \circ(\lambda s .\lfloor s\rfloor)$ : $\left[D \rightarrow S_{\perp}\right] \times\left[S_{\perp} \rightarrow D\right] \rightarrow\left[S \rightarrow S_{\perp}\right]$. We proceed similarly,

$$
\begin{aligned}
& \left(c^{\prime} \circ \lambda(h, k) .(H(h), K(k))\right)(h . k) \\
& =\lambda \text { s.case } s \text { of }\left\{\begin{aligned}
j^{-1}\left(i n_{1}(a)\right) & \left.\rightarrow\left\lfloor j^{-1}\left(i n_{1}(a)\right)\right\rfloor\right) \\
j^{-1}\left(i n_{2}\left(a, t_{0}\right)\right) & \left.\rightarrow\left\lfloor j^{-1}\left(i n_{2}\left(a, \lambda u \cdot h\left(k\left(t_{0} *\right)\right)\right)\right)\right\rfloor\right) \\
j^{-1}\left(i n_{3}\left(t_{1}, t_{2}\right)\right) & \longrightarrow\left\lfloorj ^ { - 1 } \left(i n _ { 3 } \left(\lambda u \cdot h\left(h\left(t_{1} *\right)\right), \lambda u h(k)\right.\right.\right.
\end{aligned}\right.
\end{aligned}
$$$$
=\left(\gamma \circ c^{\prime}\right)(h, k) \begin{array}{r}
\left.j^{-1}\left(i n_{3}\left(t_{1}, t_{2}\right)\right) \rightarrow\left\lfloor j^{-1}\left(i n_{3}\left(\lambda u . h\left(k\left(t_{1} *\right)\right), \lambda u . h\left(k\left(t_{2} *\right)\right)\right)\right)\right\rfloor\right) \\
\text { (by strictness of } h \text { and } k)
\end{array}
$$

By Lemma 36 and the minimal invariant property of $(S, j)$,

$$
i_{D S} \circ i_{D S}^{-1} \circ(\lambda s .\lfloor s\rfloor)=c^{\prime}(\operatorname{fix}(\lambda(h, k) .(H(h), K(k))))=\operatorname{fix}(\gamma)=\lambda s .\lfloor s\rfloor .
$$

Thus, $i_{D S}: D \cong S_{\perp}$ is indeed an isomorphism.

As before, the fixed point equation $i_{D S}=H\left(i_{D S}, i_{D S}^{-1}\right)$ immediately yields the remainder of the lemma.

Lemma 38 establishes Lemma 26. 


\section{References}

[AJ04] Klaus Aehlig and Felix Joachimski. Operational aspects of untyped normalization by evaluation. Mathematical Structures in Computer Science, 14:587-611, August 2004.

[Bar84] Henk P. Barendregt. The Lambda Calculus: Its Syntax and Semantics, volume 103 of Studies in Logic and the Foundations of Mathematics. North-Holland, revised edition, 1984.

[BS91] Ulrich Berger and Helmut Schwichtenberg. An inverse of the evaluation functional for typed $\lambda$-calculus. In Proceedings of the Sixth Annual IEEE Symposium on Logic in Computer Science, pages 203-211, Amsterdam, The Netherlands, July 1991.

[CD97] Thierry Coquand and Peter Dybjer. Intuitionistic model constructions and normalization proofs. Mathematical Structures in Computer Science, 7:75-94, 1997.

[Fil99] Andrzej Filinski. A semantic account of type-directed partial evaluation. In G. Nadathur, editor, International Conference on Principles and Practice of Declarative Programming, volume 1702 of Lecture Notes in Computer Science, pages 378-395, Paris, France, September 1999. SpringerVerlag.

[FR03] Andrzej Filinski and Henning Korsholm Rohde. A denotational account of untyped normalization by evaluation (extended version, with detailed proofs). BRICS Report RS-03-40, University of Aarhus, Denmark, December 2003. Available from http://www.brics.dk/RS/03/40/.

[FR04] Andrzej Filinski and Henning Korsholm Rohde. A denotational account of untyped normalization by evaluation. In I. Walukiewicz, editor, 7th International Conference on Foundations of Software Science and Computation Structures (FOSSACS 2004), volume 2987 of Lecture Notes in Computer Science, pages 167-181, Barcelona, Spain, March 2004. Springer-Verlag.

[GL02] Benjamin Grégoire and Xavier Leroy. A compiled implementation of strong reduction. In Simon Peyton Jones, editor, Proceedings of the Seventh ACM SIGPLAN International Conference on Functional Programming, SIGPLAN Notices, Vol. 37, No. 9, pages 235-246, Pittsburgh, Pennsylvania, September 2002. ACM Press.

[Mit96] John C. Mitchell. Foundations for Programming Languages. The MIT Press, 1996.

[MTHM97] Robin Milner, Mads Tofte, Robert Harper, and David MacQueen. The Definition of Standard ML. The MIT Press, revised edition, 1997.

[Pit93] Andrew M. Pitts. Computational adequacy via 'mixed' inductive definitions. In Mathematical Foundations of Programming Semantics, volume 802 of Lecture Notes in Computer Science, pages 72-82. Springer-Verlag, April 1993. 
[Pit96] Andrew M. Pitts. Relational properties of domains. Information and Computation, 127(2):66-90, June 1996.

[Plo77] Gordon D. Plotkin. LCF considered as a programming language. Theoretical Computer Science, 5(3):223-255, December 1977.

[SPG03] Mark R. Shinwell, Andrew M. Pitts, and Murdoch J. Gabbay. FreshML: Programming with binders made simple. In Eighth ACM SIGPLAN International Conference on Functional Programming, pages 263-274. ACM Press, Uppsala, Sweden, August 2003. 


\section{Recent BRICS Report Series Publications}

RS-05-4 Andrzej Filinski and Henning Korsholm Rohde. Denotational Aspects of Untyped Normalization by Evaluation. February 2005. $51 \mathrm{pp}$. Extended version of an article to appear in the FOSSACS 2004 special issue of RAIRO, Theoretical Informatics and Applications.

RS-05-3 Olivier Danvy and Mayer Goldberg. There and Back Again. January 2005. iii+16 pp. Extended version of an article to appear in Fundamenta Informatica. This version supersedes BRICS RS-02-12.

RS-05-2 Dariusz Biernacki and Olivier Danvy. On the Dynamic Extent of Delimited Continuations. January 2005. ii+30 pp.

RS-05-1 Mayer Goldberg. On the Recursive Enumerability of FixedPoint Combinators. January 2005. 7 pp. Superseedes BRICS report RS-04-25.

RS-04-41 Olivier Danvy. Sur un Exemple de Patrick Greussay. December 2004. 14 pp.

RS-04-40 Mads Sig Ager, Olivier Danvy, and Henning Korsholm Rohde. Fast Partial Evaluation of Pattern Matching in Strings. December 2004. 22 pp. To appear in TOPLAS. Supersedes BRICS report RS-03-20.

RS-04-39 Olivier Danvy and Lasse R. Nielsen. CPS Transformation of Beta-Redexes. December 2004. ii+11 pp. Superseedes an article to appear in Information Processing Letters and BRICS report RS-00-35.

RS-04-38 Olin Shivers and Mitchell Wand. Bottom-Up $\beta$-Substitution: Uplinks and $\lambda$-DAGs. December 2004.

RS-04-37 Jørgen Iversen and Peter D. Mosses. Constructive Action Semantics for Core ML. December 2004. 68 pp. To appear in a special Language Definitions and Tool Generation issue of the journal IEE Proceedings Software.

RS-04-36 Mark van den Brand, Jørgen Iversen, and Peter D. Mosses. An Action Environment. December 2004. 27 pp. Appears in Hedin and Van Wyk, editors, Fourth ACM SIGPLAN Workshop on Language Descriptions, Tools and Applications, LDTA '04, 2004, pages 149-168. 